Copyright

by

David Gray Lassiter

2012 
The Thesis Committee for David Gray Lassiter

Certifies that this is the approved version of the following thesis:

Effect of an Energy Drink on Physical and Cognitive Performance in Trained Cyclists

\begin{abstract}
APPROVED BY
SUPERVISING COMMITTEE:
\end{abstract}

John Ivy, Supervisor

Roger Farrar 
Effect of an Energy Drink on Physical and Cognitive Performance in Trained Cyclists

by

\author{
David Gray Lassiter, B.A.
}

\author{
Thesis \\ Presented to the Faculty of the Graduate School of \\ The University of Texas at Austin \\ in Partial Fulfillment \\ of the Requirements \\ for the Degree of
}

Master of Science in Kinesiology

The University of Texas at Austin

May 2012 


\section{Dedication}

This work is dedicated to those who have supported me outside of my graduate studies over the last few years. My family deserves as much credit as anyone for the work generated as part of this study. Namely, Kristin Bagby, Christine Lassiter, Mechelle Lassiter, Bette Rose Ryan, and Michele Poague deserve recognition; without them I would not have been able to work as tirelessly as I have on this project. 


\section{Acknowledgements}

Many individuals contributed significantly to the completion of this project. Though the thesis is my own, the project is a result of a team effort. First and foremost Lynne Kammer helped me every step of the research process, from study design, to data collection, and proofreading. Zhenping Ding played another critical role in training and overseeing the completion of the biochemistry techniques used as part of this work. Assays were completed by Zhenping Ding, Wanyi Wang, James Burns, Joowon Lee, Heontae Kim, Yi-Hung Liao, Ming Hsieh, Yoolee Kwon, Ran Hee Choi, and Kyoung Rae Kim. Day-to-day duties including supervising the study participants' exercise performance, phlebotomy and blood collection, participant randomization, and adherence to the study protocol was aided with the help of Lynne Kammer, Zhenping Ding, James Burns, Heontae Kim, Joowon Lee, Wanyi Wang, Marin Healy, Ashlee Simpson, Nathan Horney, Matthew Adamo, Jobin George, Laura Garcia, and Yang Liu. While Yang Liu is listed last, his help on this and other projects that I have been a part of is understated and I commend him for being such a hard-working and loyal individual.

My graduate studies have been made possible through the concerted efforts of the entire kinesiology staff at the University of Texas and I would like to recognize the contributions to my education made by Matthew Brothers, Edward Coyle, Hiro Tanaka, Roger Farrar, and my advisor John Ivy. Roger Farrar and John Ivy have served as readers for my thesis and for this I owe them recognition.

Lastly I would like to thank the sponsor of my research, SWSS law, and the participants who were willing to ride their bikes in the lab for the sake of expanding our scientific understanding. 


\title{
Abstract \\ Effect of an Energy Drink on Physical and Cognitive Performance in Trained Cyclists
}

\author{
David Gray Lassiter, MS KIN \\ The University of Texas at Austin, 2012
}

Supervisor: John Ivy

This study investigated the effectiveness of an energy drink (ED) in enhancing cycling time-trial performance, and cognitive performance at rest, during moderateintensity exercise, and after exercise. The protocol was double-blind, randomized, placebo-controlled, two-period, and within-subjects. The treatments were ED containing caffeine and carbohydrate, and a caffeine-free non-caloric flavored placebo beverage (PLA). Exercise performance was measured by time to finish a simulated $35 \mathrm{~km}$ timetrial course. Cognitive performance was measured by a Stroop task, a tapping task, a reaction time task, and an executive function task consisting of both tapping and reaction time. The effects of ED on blood markers were also assessed. Race performance was enhanced by an average of 3\% when participants had ED compared to PLA without a difference in rating of perceived exertion (RPE). Performance was improved by ED even in participants that arrived to the lab with elevated blood caffeine concentrations. Both before and after the exercise, ED resulted in more taps per second in the tapping task. After receiving ED, plasma insulin spiked, there was a fall in free fatty acids (FFA) and 
blood glucose remained unchanged. Exercise onset caused a drop in blood glucose when participants consumed ED, though glucose returned to a level that was not different from PLA by $29 \mathrm{~km}$ into the race. FFA also increased as the exercise continued, and were not different from PLA by $23 \mathrm{~km}$. ED elevated plasma caffeine levels. Epinephrine was elevated due to ED from $6 \mathrm{~km}$ to the end of the race. Norepinephrine was only elevated by ED at $6 \mathrm{~km}$. At rest and throughout exercise ED caused elevated lactate concentrations. When participants consumed ED they sustained a greater $\mathrm{VO}_{2}$ and heart rate throughout the race. In summary, ED enhanced exercise performance and simple movement time as assessed by the tap test before and after intense exercise. There was enough caffeine in ED to enhance physical performance without causing negative effects on cognitive function. 


\section{Table of Contents}

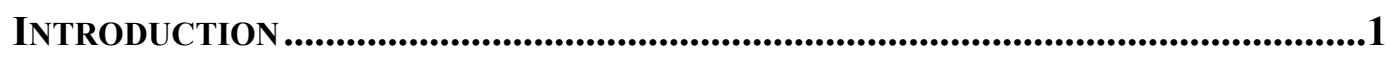

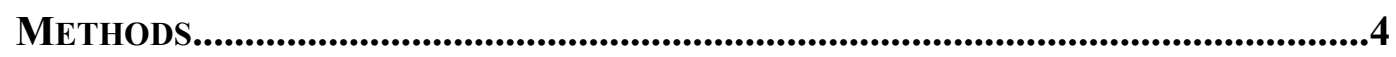

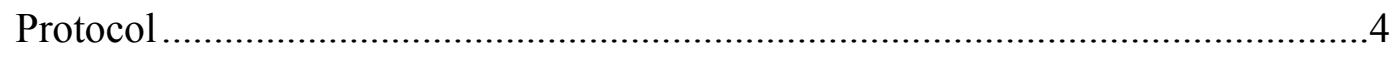

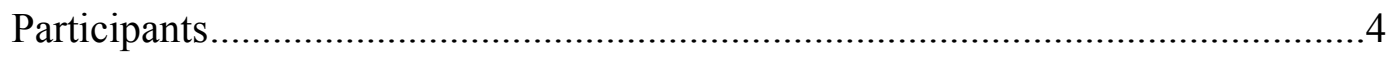

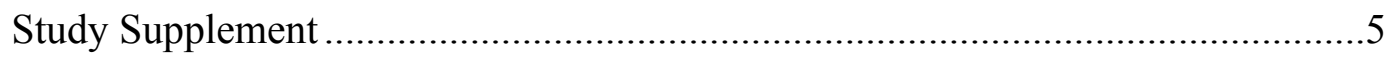

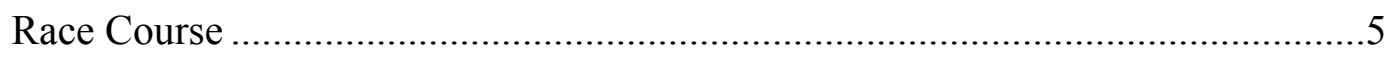

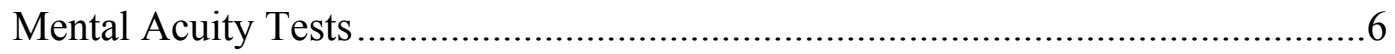

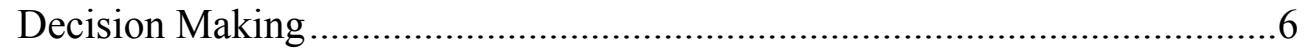

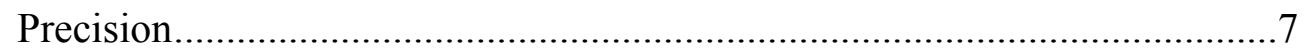

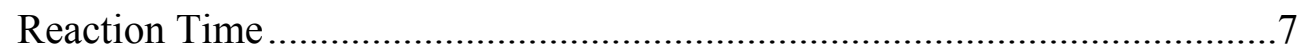

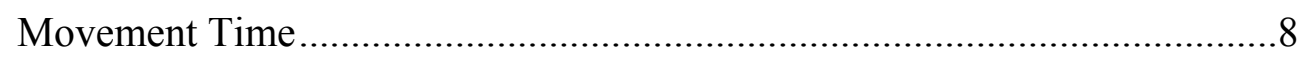

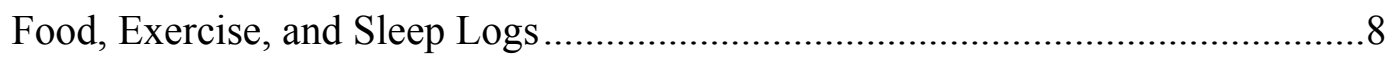

Participant Health and Safety Procedures .............................................................

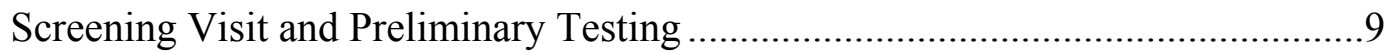

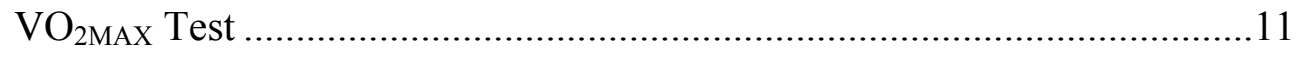

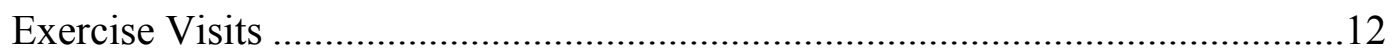

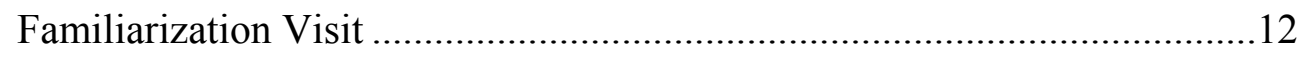

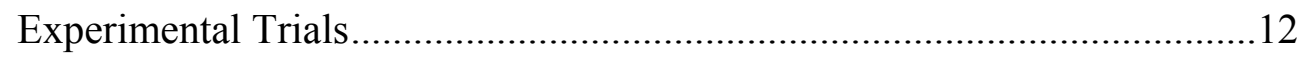

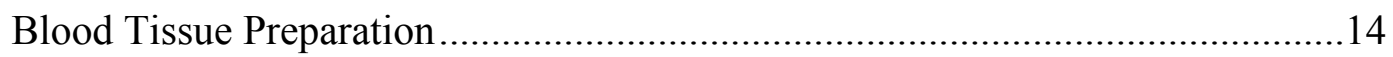

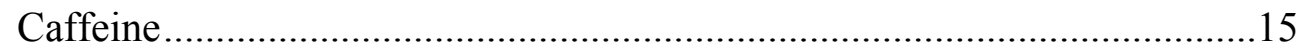

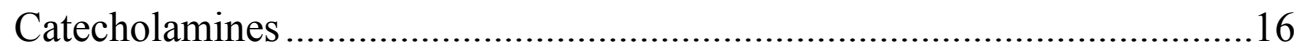

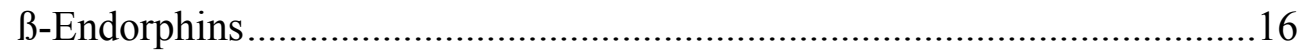

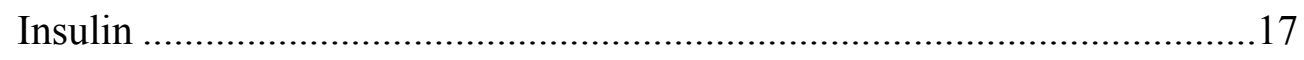

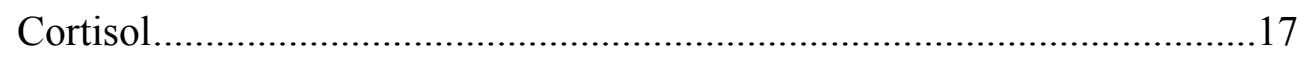

Free Fatty Acids ................................................................................... 18 


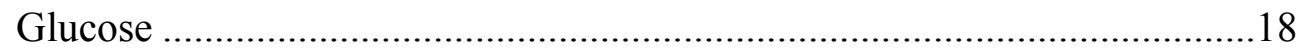

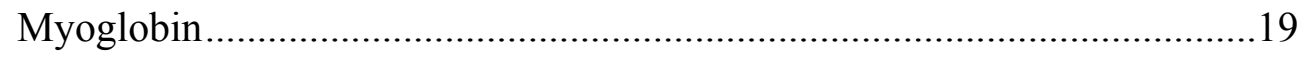

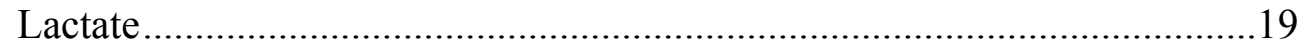

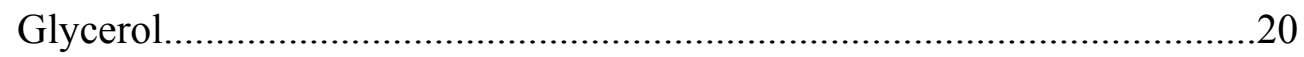

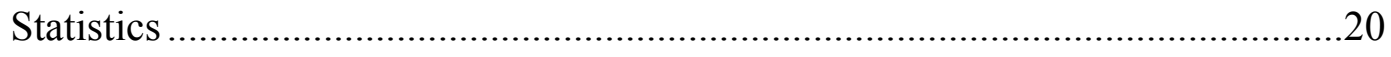

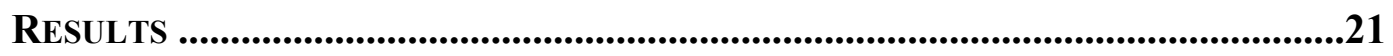

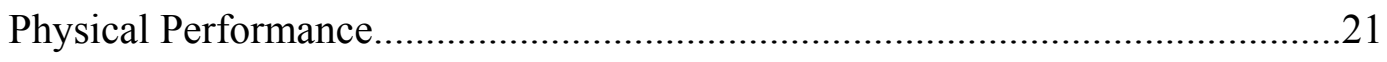

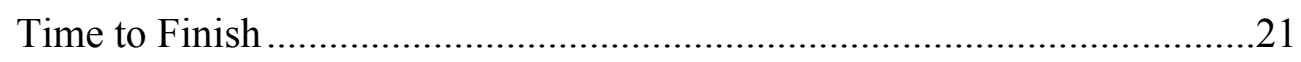

Rating of Perceived Exertion .....................................................................21

Cognitive and Psychomotor Performance ..........................................................22

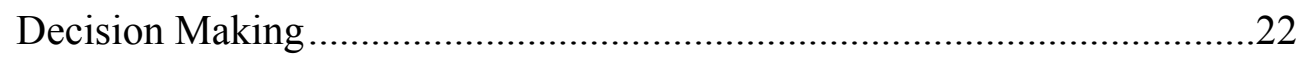

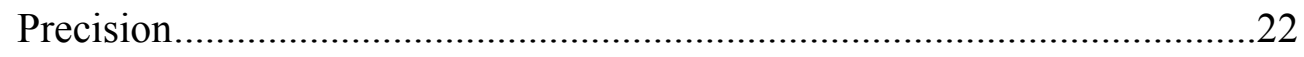

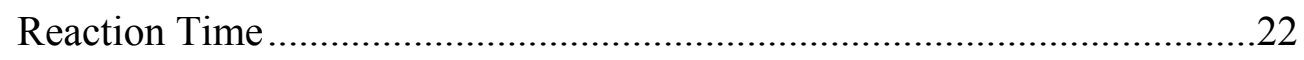

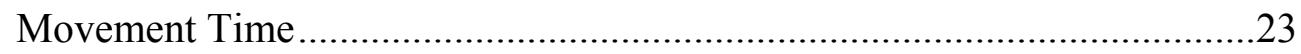

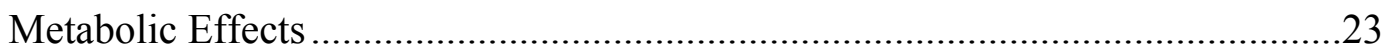

Plasma Substrates..............................................................................23

Caffeine, Catecholamines, $\beta$-endorphins and Cortisol ..................................24

Physiological indicators of work output .....................................................24

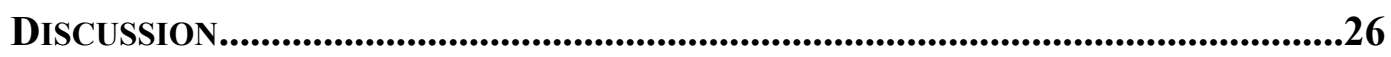

LITERATURE REVIEW ...................................................................................................34

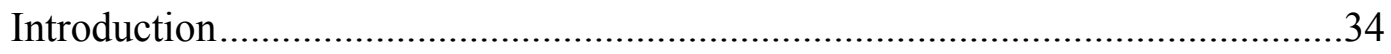

The Effects of Energy Drink Ingredients on Aerobic Performance ........................35

Caffeine Enhances Aerobic Exercise Performance …………………….........35

Carbohydrate Type and Timing Influence Effectiveness of Carbohydrate as an

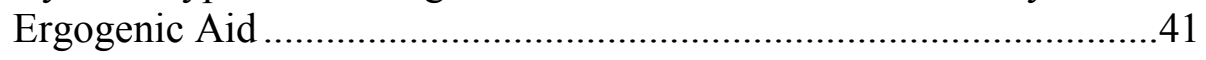

Other Energy Drink Ingredients Lack Sufficient Research to Conclude

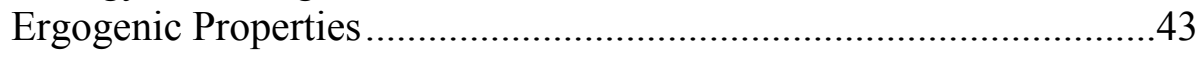

Energy Drinks Reliably Enhance Aerobic Performance ...............................48 
Conclusion on Energy Drink Ingredients Effects on Aerobic Performance

The Effects of Energy Drink Ingredients on Cognitive Performance...................50

Caffeine Enhances Various Aspects of Cognitive Function.......................50

Carbohydrate Ingestion is Associated with Improved Cognitive Performance52

Other Energy Drink Ingredients Lack Sufficient Research to Conclude

Cognition-Enhancing Properties

Energy Drinks Enhance Cognitive Performance Greater than Constituent Ingredients......................................................................56

Conclusion on Energy Drink Ingredients Effects on Cognitive Performance .......57

Aerobic Exercise Enhances Reaction Time without a Detriment to other Cognitive

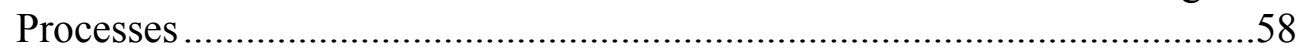

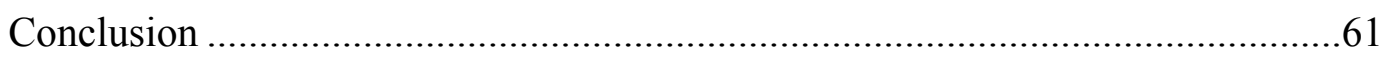

APPENDIX A: DESCRIPTIVE AND INFERENTIAL STATISTICS................................63

APPENDIX B: RAW DATA .....................................................................................73

APPENDIX C: STUDY SUPPLEMENT DETAIL .........................................................112

APPENDIX D: 35-KM COURSE DETAIL .......................................................113

APPENDIX E: FIGURES OF RESULTS .......................................................................114

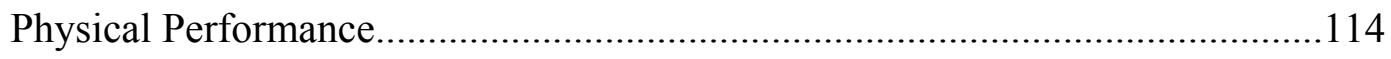

Cognitive and Psychomotor Performance .....................................................115

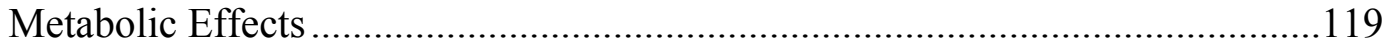

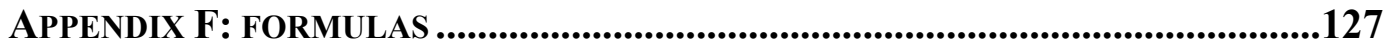

Equation 1: Estimating a Missing Data Point from an Otherwise Complete Matrix

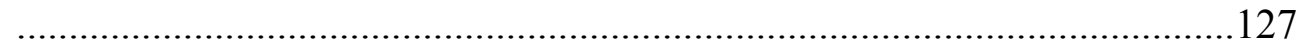

Equation 2: Estimating Maximal Heart Rate ..................................................128

Equation 3: Calculating Work Rate to Elicit a Specific Percentage of $\mathrm{VO}_{2 \mathrm{MAX}} .128$ 
APPENDIX G: EXeRCISE VISIT TIMELINE ............................................................129

REFERENCES............................................................................................................130 


\section{INTRODUCTION}

Many athletic competitions are contested in a way that makes supplementation during exercise difficult. Attempting to supplement during a cycling time-trial that is 60 minutes or less in duration is not desirable since race performance may suffer and the nutritional intervention will not have sufficient time to have full ergogenic effects. The ingestion of caffeine and carbohydrate in an energy drink have been demonstrated to have ergogenic effects when taken before exercise (Ivy et al., 2009). Additionally, energy drink consumption has been linked to enhanced reaction times and reduce reports of mental fatigue (Howard \& Marczinski, 2010). The possibility that an energy drink can enhance cognitive performance during and after intense exercise has not been wellstudied.

It has been shown that ingesting carbohydrates during prolonged aerobic activity can enhance athletic performance by increasing endurance (Coyle et al., 1986; Hulston \& Jeukendrup, 2008; Ivy et al., 1983; Welsh et al., 2002; Yaspelkis et al., 1993) or work output in a specified time (Ivy et al., 1979). Carbohydrate supplementation prior to exercise onset has also been shown effective in some studies (Ivy et al., 2009; Kirwan, et al., 1998; Sherman et al., 1989) but not all (Foster et al., 1979; Hargreaves et al., 1987; Kovacs et al., 1998; Rollo \& Williams, 2010). The difference in results among the studies can be attributed to different types of carbohydrate ingested, other ingredients in the nutritional supplements, timing of the supplementation, and the type of exercise protocol utilized.

When taken before or during exercise, caffeine exhibits ergogenic effects as demonstrated in multiple studies dating back to the early $20^{\text {th }}$ century (Costill et al., 1978; Hulston \& Jeukendrup, 2008; Ivy et al., 2009; Rivers \& Webber, 1907; Spriet et al., 
1992; Thornton et al., 1939). Traditionally doses of at least $3 \mathrm{mg}$ caffeine per $\mathrm{kg}$ of body weight have been used to elicit the ergogenic effect (Costill et al., 1978; Essig et al., 1980; Graham \& Spriet, 1991, 1995; Hulston \& Jeukendrup, 2008), though lower doses have been effective if co-ingested with carbohydrate (Alford et al., 2001; Ivy et al., 2009; Kovacs et al., 1998).

At rest, caffeine and carbohydrates have been shown to improve performance on cognitive tasks. A caffeine and carbohydrate drink has been shown to enhance cognitive vigilance (Kennedy \& Scholey, 2004), performance on rapid visual information processing, simple reaction time, and mood (Smit et al., 2004). If individuals are fasted carbohydrates and caffeine together boost performance on a letter finding task better than caffeine alone (Smit et al., 2004). Caffeine enhances simple reaction time, mood, and lowers perception of difficulty of cognitive tasks following 8 hours of caffeine abstinence (Heatherley et al., 2005).

Carbohydrate supplementation during exercise attenuates decrements in cognitive performance if the exercise is sustained over several hours (Lieberman, 2003; Lieberman et al., 2002). Memory also is enhanced when blood glucose is elevated above fasting levels (Benton \& Owens, 1993).

Exercise itself may enhance, degrade, or have no effect on cognitive performance depending on the type of exercise protocol and type of cognition evaluated (Tomporowski, 2003). Steady-state exercise, specifically, may enhance not only reaction time, but some aspects of higher-level cognitive function (Lambourne, Audiffren, \& Tomporowski, 2010a; Tomporowski, 2003). The effects appear to be transient and degrade immediately after exercise cessation (Lambourne, Audiffren, \& Tomporowski, 2010a). Memory may be enhanced by aerobic exercise as well (Coles \& Tomporowski, 2008). 
It has been demonstrated that a combination of caffeine and carbohydrate can enhance both physical and cognitive performance during cycling exercise lasting greater than 2 hours (Hogervorst et al., 2008). What has not been unequivocally demonstrated is if an ED can produce the same cognitive and physical results in a shorter duration exercise setting. Furthermore, it is not clear if an ED will continue to enhance cognitive processes after completion of such an exercise.

The purpose of this study was to investigate the effectiveness of an ED in enhancing cycling time trial performance, cognitive performance at rest, cognitive performance during moderate-intensity exercise, and cognitive performance immediately after completing a bout of moderate- to high-intensity exercise. Specifically we had the following hypotheses:

Hypothesis 1: Consumption of $480 \mathrm{ml}$ of an ED containing caffeine, carbohydrates, ginseng, glucuronolactone, guaraná, inositol, and taurine 60 minutes prior to starting a $35-\mathrm{km}$ cycling time-trial race will result in a faster race time.

Hypothesis 2: Consumption of the same ED 40 minutes prior to a set of cognitive tests including reaction time, movement time, and decision making will result in improved performance.

Hypothesis 3: Consumption of the same ED will result in improved reaction time and decision making while exercising at $70 \%$ of $\mathrm{VO}_{2 \max }$ immediately after completion of the $35-\mathrm{km}$ time-trial race.

Hypothesis 4: Consumption of the same ED will result in improved performance on reaction time, movement time, and decision making while sitting restfully immediately after completion of the $35-\mathrm{km}$ time-trial race. 


\section{METHODS}

\section{Protocol}

The study was designed to evaluate the effects of a commercially available energy drink (ED) on cycling time-trial performance. The time trial was $35 \mathrm{~km}$, was performed on a computer-controlled cycle ergometer, and lasted approximately 60 minutes for the participants to complete. The study also investigated the effects of ED on tests of mental acuity at rest, near completion of exercise, and post exercise.

The experimental protocol was a double-blind, randomized, placebo-controlled, two-period, within-subjects crossover study. The two treatments were ED containing caffeine and carbohydrate, and a caffeine-free non-caloric flavored placebo beverage (PLA). Exercise performance was measured by time-to-complete the $35 \mathrm{~km}$ time-trial course performed on a cycle ergometer. Mental acuity was measured by a Stroop task, a tapping task, a choice reaction time task, and an executive function task consisting of both tapping and choice reaction time. The effects of ED on blood metabolites and hormones at rest and during exercise were also assessed.

\section{Participants}

Fifteen ( 8 male and 7 female) trained cyclists between 20 and 45 years of age were recruited from the Austin, TX area. Volunteers were invited to participate if healthy and if the recorded $\mathrm{VO}_{2 \max }$ from a standardized ramping protocol was at least 45 $\mathrm{ml} / \mathrm{kg} / \mathrm{min}$ for women or $55 \mathrm{ml} / \mathrm{kg} / \mathrm{min}$ for men. Participants had a mean age of 31 years, mean body mass of $67.8 \mathrm{~kg}$ and $56.9 \mathrm{ml} / \mathrm{kg} / \mathrm{min}$ was the mean $\mathrm{VO}_{2 \mathrm{MAX}}$. Complete participant characteristics are described in the appendix. 


\section{Study Supplement}

The 2 beverages were $480 \mathrm{ml}$ of ED (160 mg caffeine, $54 \mathrm{~g}$ carbohydrate) and $480 \mathrm{ml}$ of PLA (caffeine-free non-caloric similarly-flavored placebo). The beverages were provided by SWSS Law (Corona, CA). A detailed list of ingredients for each drink can be found in the appendix. Each participant was randomly assigned to receive 1 of the drinks at his/her first experimental trial and the other drink at the second experimental trial at least 6 days later. The randomization procedure resulted in 8 participants receiving ED at the first experimental trial, while the other 7 participants received it at the second experimental trial. Beverages were served cold and participants were instructed to ingest them as quickly as possible without causing stomach discomfort. A single participant received the drinks at room temperature at her explicit request.

\section{Race Course}

The course was $35 \mathrm{~km}$ long and was programmed using Velotron 3D software (Version 3) that coordinated with the cycle ergometer (Racermate, Seattle, WA, USA). The course had flat portions to correspond with data collection at $0.75-1.25,5-8,16-18$, and $28-30 \mathrm{~km}$. Hills of $5.4 \%$ grade were placed at $11.75-12.25,22.75-23.25$, and $33.75-$ $34.25 \mathrm{~km}$. The rest of the course was randomly created with no graded portion exceeding an absolute value of $3 \%$. The net grade over the $35-\mathrm{km}$ course was $1 \%$ uphill. The ergometer was equipped with a toggle to allow the participants to shift gears at will to adjust to the terrain. The details of the course are more specifically described in the appendix. 


\section{Mental Acuity Tests}

There were 4 tests of mental acuity. The mental acuity tests were given at 3 times: before consumption of the test beverage (Pre Drink, Pre Race), after beverage consumption/before exercise (Post Drink, Pre Race), and after exercise completion (Post Race). Each test measured a different aspect of mental acuity. The tests included a tapping task, choice reaction time, an executive function test (a choice reaction time test including "go" and "no-go" signals while participants simultaneously tap with their other hand), and a Stroop task that included two stimulus types. The Stroop task was implemented on a computer using Stroop Research software (Life Science Associates, Bayport, NY, USA). All other mental acuity tasks were conducted on a MOART reaction and movement time panel (Lafayette Instrument, Lafayette, IN, USA).

\section{DECISION MAKING}

The Stroop test presented color words on a computer screen (e.g., "red", "green", or "blue") in two ways to assess decision making: "incongruent" and "read". In "incongruent", the words on the computer screen were presented in a font color that was incongruent with the word definition (e.g., the word "red" may be presented in a blue font). Correct responses were associated with a key corresponding to the font color. Alternatively, in "read", the color word would appear with a box around it, and the correct response was associated with a key corresponding to the word definition. See the examples below. Decision making was assessed by monitoring how frequently errors were made.

"Incongruent" presentation type RED
"Read" presentation type

RED 
In the executive function test, participants tapped a button continuously with one hand while their other hand was holding down another "home" button. The non-tapping hand continued to hold the "home" button down until a red or green light appeared over another "movement" button. If the light was red, the participant moved to the corresponding "movement" button, but if the light was green the participant kept his or her hand on the "home" button while continuing to tap with the other hand. Decision making was assessed by the error rate. Every participant began with the right hand assigned to the "home" button and the left hand assigned to the "tap" button. In an effort to control for hand dominance, after five repetitions of the task, participants switched hand assignments and repeated the task (i.e. left hand was assigned to the "home" button and the left hand was assigned to the "tap" button). The process was repeated twice more so that a total of ten repetitions were recorded with each hand at the "home" button.

\section{Precision}

We assessed precision by instructing participants to hit the center of a button on the reaction time board in the tapping test as well as in the reaction time test. If a participant hit the button but not in the center, the hit was counted as an "outer rim" contact. Precision was assessed by the percentage of total taps which did not hit the outer rim.

\section{REACTION TIME}

Three tests were used to assess reaction time: Stroop, executive function and reaction time. The Stroop test and executive function test have been described. In the reaction time test participants were instructed to put their finger on a "home" button and keep it there until a green light appeared over another button. As soon as the green light appeared, participants moved their finger off of the "home" button and to the 
"movement" button as quickly as possible. Reaction time was defined as the amount of time that it took a participant to remove their finger from the "home" button after a red light appeared during the executive function test.

\section{Movement Time}

Movement time was measured in two ways: simple and gross. Simple movement refers to tapping speed in the tap and executive function tests; more taps per second is a result of an improvement in movement speed. Gross movement was assessed in the reaction time and executive function tests as how quickly participants moved to the "movement" button after removing their finger from the "home" button.

\section{Food, Exercise, and Sleep Logs}

Participants were given a log to record their habits for the 48 hours before the exercise visits (familiarization visit and experimental trials). The participants recorded all food ingested in the 24 hours before the exercise visits and were instructed to abstain from alcohol and caffeine. Additionally participants recorded exercise and sleep habits in the 48 hours prior to the exercise visits. The participants completed the logs prior to the familiarization visit in order to verify correct completion of the records, but the data was not analyzed. If the logs submitted by the participants at the familiarization visit indicated low energy intake ( $<1800 \mathrm{kcal} / 24$ hours), low carbohydrate intake ( $<225 \mathrm{~g} / 24$ hours), high exercise ( $>3$ hours of moderate intensity/48 hours), or low sleep $(<14$ hours of moderate quality/48 hours) then the participants were instructed to change their habits accordingly in the 2 days before the first experimental trial. If participants reported to the first experimental trial with values outside of these acceptable ranges, the trial was rescheduled: only one participant had to reschedule due to inadequate sleep. 


\section{Participant Health and Safety Procedures}

At each visit to our lab after the screening visit participants reported any newly occurring illnesses, diseases, or injuries. An Adverse Event Form was completed by the investigators in these circumstances. Of the adverse events reported, none were thought to be related to the treatment drinks. A male participant was dropped from the study after partial completion after he suffered injuries in a bicycle wreck unrelated to the research study. Another male participant reported knee pain that he attributed to the cycle ergometer used in the research: the investigators offered to reschedule the participant's experimental trials to allow his pain to subside, but he claimed the knee pain did not affect his exercise performance since it only onset approximately 24 hours after he completed the time-trial rides.

\section{Screening Visit and Preliminary Testing}

The study was approved by the Institutional Review Board at The University of Texas at Austin prior to any participant recruitment or screening procedures. After an initial contact by telephone or email, a volunteer was invited to participate in a screening visit. At the screening visit a detailed explanation of the experimental procedures and the potential risks of the study were given both verbally and in writing to all potential participants. Volunteers were given the opportunity to ask questions before signing the informed consent, according to the protocol described in the University of Texas at Austin's 'Institutional Review Board Procedures Manual for Faculty, Staff and Student Researchers with Human Subjects'. All volunteers gave informed consent in writing prior to any biomedical research project-specific procedures.

After the informed consent was signed, the volunteer completed the Health Screener. The volunteer was only invited to participate in the study if he/she answered 
"no" to questions on the Health Screener pertaining to hypertension, circulatory, liver and kidney conditions, pregnancy and diabetes. Nutritional supplements were acceptable only if the volunteers agreed to discontinue use for at least 1 week prior to the follow-up visit and would maintain abstinence throughout the duration of the study. Prescription medications were acceptable only if the dosage had been constant for at least 2 months leading up to the screening.

After the Health Screener the volunteer completed the Study Screener. Volunteers were permitted to proceed with the screening visit if their reported weekly cycling exercise was at least 3 hours of moderate intensity. Each hour of low-intensity or highintensity cycling exercise was considered equivalent to 0.5 or 1.5 hours of moderateintensity cycling exercise. Volunteers were permitted to proceed with the screening visit if their reported daily caffeine consumption was approximately $200 \mathrm{mg}$ or less: volunteers that willingly agreed to reduce daily consumption to meet this requirement were also permitted to continue with the screening visit.

After finishing the Study Screener the volunteer submitted to a blood-pressure recording. If the volunteer had systolic blood pressure in excess of $140 \mathrm{mmHg}$ or diastolic blood pressure in excess of $90 \mathrm{mmHg}$ the volunteer was not permitted to participate in the study.

The volunteer then completed a brief intelligence test, the Kaufman Brief Intelligence Test (KBIT2) (Psychcorp, San Antonio, TX, USA). The results of the test were not used for screening purposes and this was explained to volunteers to help attenuate anxiety.

Finally the volunteer performed a ramping exercise protocol on a Velotron cycle ergometer (RacerMate, Seattle, WA, USA) while breathing into a TrueOne 2400 metabolic cart (ParvoMedics, Sandy, UT, USA) in order to assess $\mathrm{VO}_{2 \max }$. Male 
volunteers with a $\mathrm{VO}_{2 \mathrm{MAX}}$ of at least $55 \mathrm{ml} / \mathrm{kg} / \mathrm{min}$ were invited to participate in the study while the minimum qualifying $\mathrm{VO}_{2 \max }$ for females was $45 \mathrm{ml} / \mathrm{kg} / \mathrm{min}$.

If the volunteers satisfied all of the criteria he/she was invited to participate in the study and the remaining visits were scheduled.

\section{VO $_{2 \text { MAX }}$ TEST}

The $\mathrm{VO}_{2 \mathrm{MAX}}$ test required volunteers to maintain a cadence of at least $60 \mathrm{RPM}$ on a cycle ergometer for as long as possible while the resistance on the ergometer continued to increase over time. Specifically, volunteers worked at a power output of 100 Watts for the first 2 minutes after which the resistance increased. For the first 10 minutes, the power output increased every 2 minutes by 50 Watts and 25 Watts for men and women, respectively. After 10 minutes of the ramp protocol, the power output continued to increase by 25 Watts every minute for both genders. When volunteers reached a work rate where a cadence of 60 RPM could not be maintained, the test was ended and participants were immediately put into a low-resistance cool-down ride. A single participant ended the test by his own volition due to reported difficulty in breathing through the Daniel's valve: all other participants whose data appear in the results ended the $\mathrm{VO}_{2 \mathrm{MAX}}$ test when the appropriate cadence could no longer be maintained. Throughout the ramp test volunteers' gas exchange was analyzed by a metabolic cart. By the end of the $\mathrm{VO}_{2 \mathrm{MAX}}$ test all participants had reached a heart rate (HR) within $10 \%$ of $\mathrm{HR}_{\mathrm{MAX}}$ as predicted by the formula proposed by Tanaka et al. (2001), had a respiratory exchange ratio (RER) above 1.10 , and had a final $\mathrm{VO}_{2}$ recording that was not maximal (indicating $\mathrm{VO}_{2}$ had already plateaued). For the exercise visits participants rode at $50 \%$ of $\mathrm{VO}_{2 \mathrm{MAX}}$ for a warm-up ride and at $70 \%$ of $\mathrm{VO}_{2 \mathrm{MAX}}$ during the post-race Stroop task. The 
Wattages necessary to elicit the appropriate $\mathrm{O}_{2}$ consumption were calculated using the formula from Åstrand and Rodahl (1977).

\section{Exercise Visits}

\section{FAMILIARIZATION VISIT}

The familiarization visit was identical to the experimental trial visits except that no treatment drink was given to the participant and blood was not drawn. The participant completed baseline mental acuity tests upon arrival to the lab. The participant then consumed a volume of water equal the treatment drink volume $(480 \mathrm{ml})$. The participant repeated the mental acuity tests 40 minutes after water consumption then engaged in a warm-up exercise at $50 \%$ of $\mathrm{VO}_{2 \mathrm{MAX}}$, a $35 \mathrm{~km}$ time-trial ride, a 2 min ride at $70 \%$ of $\mathrm{VO}_{2 \mathrm{MAX}}$ while completing a Stroop task, and post-exercise mental acuity tests as outlined for the experimental trials below. Body mass, HR, RPE, and respiratory gas collection were gathered in identical fashion as the experimental trials. Water was provided to participants at the same regular intervals as in the experimental trials. The volume of water provided at the familiarization was a standard $800 \mathrm{~mL}$, however participants received more water at the experimental trials to offset fluid loss experienced during the familiarization trial. For example, if a participant lost $1 \mathrm{~kg}$ of body mass during the familiarization, he/she was provided with $1800 \mathrm{~mL}$ of total water during each experimental trial $(1000 \mathrm{~mL}$ in addition to the $800 \mathrm{~mL}$ at familiarization). After completion of the post-exercise mental acuity tests, the participants were provided a light meal.

\section{EXPERIMENTAL TRIALS}

The only difference between the experimental trials and the familiarization visit is that participants received the treatment drinks at the experimental trials and blood 
samples were drawn at several times. The familiarization visit and experimental trials consisted of three blocks, pre time trial, time trial, and post time trial. The timeline for these visits can be found in the appendix.

\section{Pre Time Trial}

The participant was fitted with a heart-rate monitor (Polar RS400, Finland), weighed after urination, and completed a bout of mental acuity tests (described below). During the mental acuity tests resting HR was recorded. After the tests, the participant was equipped with a forearm intravenous catheter then blood was drawn. The participant ingested the treatment drink and rested for 40 minutes. After resting, the participant completed another bout of mental acuity tests after which a second blood sample was drawn. The participant used the restroom if needed then completed a 5-minute warm-up ride at $50 \% \mathrm{VO}_{2 \mathrm{MAX}}$.

\section{Time Trial}

Immediately after the warm-up ride, the participant began the customized $35-\mathrm{km}$ time trial (described in detail later). Throughout the time-trial ride the participant received a pre-determined volume of water throughout the time-trial ride to offset sweat loss.

In addition to the 2 samples taken prior to exercise, blood was drawn at 5 times during the time-trial ride. Specifically blood was sampled when the participant had reached $6,17,23,29$, and $34 \mathrm{~km}$ of the course.

Ventilation, $\mathrm{VO}_{2}, \mathrm{CO}_{2}$ production and $\mathrm{RER}$ were recorded at 3 times in the ride using the same metabolic system used for the $\mathrm{VO}_{2 \mathrm{MAX}}$ test. Gas collection began when the participant crossed 5, 16, and $28 \mathrm{~km}$ and continued for 4 minutes. The course was designed so that these periods of gas collection were during flat race sections so that the 
participants could achieve steady state. The full 4 minutes of data was used in data analysis unless there was a delay in starting data collection, in which case all data up to 4 minutes was analyzed.

HR was recorded at several points during the $35-\mathrm{km}$ course. Subjective rating of perceived exertion (RPE) on a Borg scale (unit-less scale ranging from 6 to 20) was obtained during exercise at the same times as HR. HR and RPE were collected at 1, 6, 12, 17, 23, 29, and $34 \mathrm{~km}$. The 12, 23, and $34 \mathrm{~km}$ markers were taken while participants were riding up a simulated hill at a $5.4 \%$ grade.

\section{Post Time Trial}

At the conclusion of the $35-\mathrm{km}$ time trial, the participant began a 2 -minute ride at $70 \% \mathrm{VO}_{2 \mathrm{MAX}}$ while simultaneously completing the Stroop task. Immediately after the Stroop task the participant completed the post-exercise mental acuity tests. The participant's HR was recorded at the start and every 30 seconds of the Stroop task. After completion of the mental acuity tests, the participant's HR was recorded once more before the catheter was removed. The participant was weighed after urinating and was finally provided with a light meal. The heart-rate monitor was retrieved and instructions for the next trial were given to the participant.

The time separating the experimental trials ranged from 6 to 21 days. The only difference between the first and second experimental trial was that the participant received the alternative treatment drink at the second experimental trial.

\section{Blood Tissue Preparation}

Each blood sample was mixed with EDTA (24 mg/ml, $\mathrm{pH} 7.4)$ in a ratio of $3 \mathrm{ml}$ blood:0.23 ml of EDTA. A $0.5 \mathrm{ml}$ sample of this anticoagulated blood was transferred to

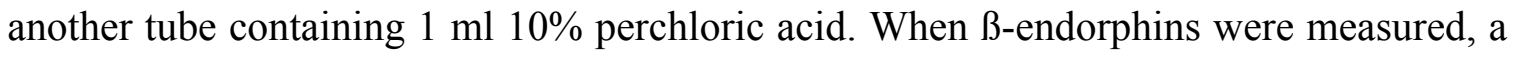


$3 \mathrm{ml}$ blood sample was added to $0.23 \mathrm{ml}$ of EDTA and $0.18 \mathrm{ml}$ of aprotinin $(10.12$ units/ml) from bovine lung (Sigma-Aldrich, St. Louis, MO, USA). All tubes were centrifuged for 10 minutes at 3,000 rpm with a JS-7.5 rotor in a Beckman J2-21 centrifuge. Plasma and perchloric acid extracts were transferred and stored at $-80^{\circ} \mathrm{C}$ for further analysis. Plasma samples were analyzed for caffeine, insulin, glucose, cortisol, human $\beta$-endorphins, catecholamines, myoglobin and free fatty acids (FFA). Perchloric acid extracts were analyzed for glycerol and lactate.

\section{CAFFEINE}

Caffeine concentration was determined for the first blood draw (Pre Drink, Pre Race), the second blood draw (Post Drink, Pre Race), and the seventh blood draw (34 km). Caffeine was analyzed using a commercially available ELISA kit (Neogen Corporation, Lexington, KY, USA). The assay worked via competitive binding to a microwell. The microwells had a coat of antibodies bound to them which were specific for caffeine or a drug-enzyme conjugate that was provided in the kit. The sample and drug-enzyme conjugate were added to the microwells and incubated. After incubation the microwells were washed to remove unbound caffeine and drug-enzyme conjugates. KBlue Substrate (TMB) was added which was recognized by the drug-enzyme conjugate. After another 30 minutes of incubation Red Stop Solution was added. The microwells were read visually at $450 \mathrm{~nm}$ on a ELx800 microplate reader (Biotek, Winooski, VT, USA). Since only the drug-enzyme conjugate recognized the TMB, the presence of caffeine resulted in less blue color development. When duplicates were tested, they were tested within the same assay (intra-assay). The average coefficient of variation (CV) for intra-assay duplicates was $3.7 \%$. 


\section{Catecholamines}

The catecholamines epinephrine and norepinephrine were analyzed from the first blood draw (Pre Drink, Pre Race), the second blood draw (Post Drink, Pre Race), the fourth blood draw $(17 \mathrm{~km})$, the sixth blood draw $(29 \mathrm{~km})$, and the seventh blood draw (34 $\mathrm{km})$. The catecholamines were analyzed using a commercially available ELISA kit (Rocky Mountain Diagnostics, Colorado Springs, CO, USA). The catecholamines were first extracted from the plasma samples using a cis-diol-specific affinity gel, acylated and then derivatized enzymatically. The extracts were then subjected to the assay. The assay worked via competitive binding to a microwell. The catecholamines and an analyte supplied with the kit competed for a limited number of sites within the microwell. After incubation, unbound catecholamines and analytes were washed away. An antibody bound to the analyte was recognized by an anti-rabbit antibody which then reacted with TMB. A stop solution was used and quantification of color development was performed at $450 \mathrm{~nm}$ by a ELx800 microplate reader (Biotek, Winooski, VT, USA). When duplicates were tested, they were tested within the same assay (intra-assay). The average CV for intraassay duplicates was $2.7 \%$ for epinephrine and $3.8 \%$ for norepinephrine.

\section{B-ENDORPHINS}

$\beta$-endorphins were analyzed from the first blood draw (Pre Drink, Pre Race), the second blood draw (Post Drink, Pre Race) and the seventh blood draw (34 km). Prior to analysis, $\beta$-endorphins were extracted using a Strata well plate (Phenomenex, Torrance, CA, USA) and dried under nitrogen using a SpedVac (Savang Instruments, Farmingdale, NY, USA) and resuspended. $\beta$-endorphins were then analyzed using a commercially available ELISA kit (MD Bioproducs, St. Paul, MN, USA). The microplates provided in the kit were pre-coated with a secondary antibody with non-specific binding sites 
blocked. A primary antibody was added and the primary antibody was bound by either biotinylated peptide or the $\beta$-endorphins in the sample. The biotinylated peptide reacted with streptavidin-horseradish peroxidase (SA-HRP) in order to catalyze TMB to produce a blue solution. A stop solution of hydrogen chloride $(\mathrm{HCl})$ was used to convert the blue to yellow color, and the intensity of the yellow color was read at $450 \mathrm{~nm}$ by a ELx800 microplate reader (Biotek, Winooski, VT, USA). When duplicates were tested, they were tested within the same assay (intra-assay). The average CV for intra-assay duplicates was $2.7 \%$.

\section{INSULIN}

Insulin was analyzed from all blood draws (Pre Drink, Pre Race; Post Drink, Pre Race; 6 km; 17 km; 23 km; 29 km; and 34 km) using a commercially available RIA kit (MP Biomedicals, Solon, OH, USA). An antibody for insulin was bound to the inside of a polypropylene tube. A radioactive form of insulin containing ${ }^{125} \mathrm{I}$ competed with the insulin from plasma samples for binding to the limited number of antibody sites within the tube. A 1470 Wallac Wizard Gamma Counter (PerkinElmer, San Jose, CA, USA) was

used to determine the amount of binding of insulin- ${ }^{125} \mathrm{I}$ and a standard curve was used to determine the concentration of insulin in the plasma sample. When duplicates were tested, they were tested within the same assay (intra-assay). The average CV for intraassay duplicates was $1.5 \%$.

\section{CORTISOL}

Cortisol was analyzed from all blood draws (Pre Drink, Pre Race; Post Drink, Pre Race; 6 km; 17 km; 23 km; 29 km; and 34 km). Cortisol was analyzed by a commercially available RIA kit (MP Biomedicals, Solon, OH, USA). An antibody for cortisol was bound to the inside of a polypropylene tube. A radioactive form of cortisol containing ${ }^{125} \mathrm{I}$ 
competed with the cortisol from plasma samples for binding to the limited number of antibody sites within the tube. A 1470 Wallac Wizard Gamma Counter (PerkinElmer, San Jose, CA, USA) was used to determine the amount of binding of cortisol- ${ }^{125}$ I and a standard curve was used to determine the concentration of cortisol in the plasma sample. When duplicates were tested, they were tested within the same assay (intra-assay). The average $\mathrm{CV}$ for intra-assay duplicates was $1.9 \%$.

\section{Free FATTY ACIDS}

FFA were analyzed from all blood draws (Pre Drink, Pre Race; Post Drink, Pre Race; $6 \mathrm{~km} ; 17 \mathrm{~km} ; 23 \mathrm{~km} ; 29 \mathrm{~km}$; and $34 \mathrm{~km}$ ) using a microfluorometric assay first outlined by Duncombe (1964) but then modified by Noma et al. (1973). FFA were extracted from plasma using chloroform, heptain, and methanol solution (CHM) and a copper reagent. The extract containing copper-bound FFA was mixed with a color reagent (22 $\mathrm{mg}$ diethyldithiocarbamate (DETC) and $10 \mathrm{ml}$ butanol) in a ratio of 1:5. The sample was then read at $436 \mathrm{~nm}$ on a Beckman DU 640 spectrophotometer (Beckman, Brea, CA, USA) after 10 minutes of incubation. When duplicates were tested, they were tested within the same assay (intra-assay). The average CV for intra-assay duplicates was $4.9 \%$.

\section{GLUCOSE}

Glucose was analyzed for all blood draws (Pre Drink, Pre Race; Post Drink, Pre Race; 6 km; 17 km; 23 km; 29 km; and 34 km).using a Glucose Color Reagent kit (Cliniqa, San Marcos, CA, USA) incorporating a modified Trinder reaction. Samples and standards reacted with the reagent provided by the kit to form a red-violet quinone, which was visualized at $500 \mathrm{~nm}$ by a Beckman DU 640 spectrophotometer. When duplicates 
were tested, they were tested within the same assay (intra-assay). The average CV for intra-assay duplicates was $0.7 \%$.

\section{MYOGLOBIN}

Myoglobin was analyzed from all blood draws except $17 \mathrm{~km}$ (Pre Drink, Pre Race; Post Drink, Pre Race; 6 km; 23 km; 29 km; and 34 km). Myoglobin was determined by a solid phase ELISA using a commercially available kit (BioCheck, Foster City, CA, USA). An antibody specific for myoglobin was fixed to the inside of wells in a microplate. The samples and standards were added, incubated, and unbound sample was washed away. Another antibody specific for myoglobin was added, incubated, then unbound portions were washed away. The second antibody contained horseradish peroxidase so the addition of TMB resulted in evolution of a blue color. After a stop reaction, the color was read at $450 \mathrm{~nm}$ by a ELx800 microplate reader (Biotek, Winooski, VT, USA). When duplicates were tested, they were tested within the same assay (intraassay). The average $\mathrm{CV}$ for intra-assay duplicates was $8.3 \%$.

\section{LACTATE}

Lactate was analyzed from the perchloric acid extracts from all blood draws (Pre Drink, Pre Race; Post Drink, Pre Race; 6 km; 17 km; 23 km; 29 km; and 34 km). Blood lactate was determined by enzymatic analysis according to Hohorst in Methods of enzymatic analysis (pp. 266-270, 1963). Samples and standards reacted with nicotinamide adenine dinucleotide (NAD) in the presence of lactate dehydrogenase (LDH) to form pyruvate and $\mathrm{NADH}$. The pyruvate was reacted with hydrazine irreversibly to form pyruvate hydrazone. The NADH was visualized at $340 \mathrm{~nm}$ by a Beckman DU 640 spectrophotometer. When duplicates were tested, they were tested within the same assay (intra-assay). The average $\mathrm{CV}$ for intra-assay duplicates was $0.8 \%$. 


\section{GLYCEROL}

Glycerol was analyzed from the perchloric acid extracts from all blood draws (Pre Drink, Pre Race; Post Drink, Pre Race; 6 km; 17 km; 23 km; 29 km; and 34 km). Blood glycerol was determined by enzymatic analysis as specified by Weiland in Methods of enzymatic analysis (pp. 1404-1409, 1974). The samples and standards were reacted with NAD and ATP in the presence of glycerol kinase (GK) and glycerol-3-phosphate dehydrogenase $(\mathrm{GPDH})$. The measured product of the reaction was NADH, which was visualized at $340 \mathrm{~nm}$ by a Beckman DU 640 spectrophotometer. When duplicates were tested, they were tested within the same assay (intra-assay). The average CV for intraassay duplicates was $6.2 \%$.

\section{Statistics}

Data were analyzed using a two-factor (treatment-by-time) repeated-measures ANOVA using SPSS version 19 (IBM, Armonk, NY, USA). Where interactions or main effects were observed, pairwise comparisons were made and differences were considered significant at $\mathrm{p}<.05$ using the least significant difference (LSD) post-hoc test. Data are expressed as means \pm standard error of the mean. 


\section{RESULTS}

All descriptive statistics and raw data used for inferential statistics are reported in the appendix. Figures were generated when statistically significant results were found and are also provided in the appendix.

\section{Physical Performance}

\section{TIME TO FINISH}

A significant treatment effect was found as every participant finished the $35-\mathrm{km}$ time trial in a shorter time after consuming ED as compared to PLA. The average improvement was $2.0 \pm 1.3$ minutes. This was an average of $3 \%$ improvement in time trial performance.

Of the 15 participants, 5 arrived to the laboratory with baseline blood caffeine concentrations of greater than $1000 \mathrm{ng} / \mathrm{ml}$ at both experimental trials. All participants claimed to have followed the instructions to abstain from caffeine for at least 24 hours. Data was split so that the participants with high-baseline caffeine levels at both experimental trials were analyzed separately than those with low-baseline caffeine levels. The effects of ED on exercise performance were unchanged, such that regardless of baseline caffeine levels, ED resulted in an average time-trial performance improvement of $3 \%$. Furthermore, regardless of baseline caffeine levels, blood caffeine elevated after consumption of the ED $(379 \mathrm{ng} / \mathrm{ml}$ to $2324 \mathrm{ng} / \mathrm{ml}$ and $1842 \mathrm{ng} / \mathrm{ml}$ to $3196 \mathrm{ng} / \mathrm{ml}$ in the low and high baseline groups, respectively).

\section{Rating Of Perceived Exertion}

There was no difference in RPE between trials. RPE was significantly increased throughout the trials regardless of treatment. 


\section{Cognitive and Psychomotor Performance}

\section{DECISION MAKING}

No effects of time or treatment were found on decision making ability in the Stroop test or executive function test.

\section{Precision}

There was no difference in precision as assessed by the reaction time test. A main effect for treatment was seen for precision when assessed by the tap test, with ED resulting in less precision than PLA. While the difference is statistically significant, the difference is not clinically relevant as it was less than $1 \%$.

\section{REACTION TIME}

There was no change in reaction time due to ED as assessed by the reaction time or Stroop tests. When examining total time to complete the executive function test (including reaction time and movement time), a main effect for treatment was found though there was no interaction of time by treatment. Since no time by treatment interaction was found, and participants were faster even before receiving ED (see the "Pre Drink, Pre Race" time), it cannot be concluded that the ED caused faster performance on the test; participants may have been performing the task faster on the day they received ED due to uncontrolled confounding variables.

Participants had faster reaction times on the Stroop test after completing the 35$\mathrm{km}$ race as demonstrated by a main effect for time. The main effect for time was found for the "incongruent" stimuli but not the "read" stimuli. Since these were the only two stimuli types in the Stroop test, the overall main effect is due to the improvement in performance on the "incongruent" stimuli specifically. 


\section{Movement Time}

A treatment-by-time interaction was found for simple movement time as assessed by the tap test. A main effect for treatment was also found. ED resulted in more taps per second compared to PLA both before exercise started and after the $35-\mathrm{km}$ time trial. The data was further analyzed by splitting the participants into separate groups for low- and high-baseline caffeine. It was found that there were no statistically significant effects for the high-baseline caffeine group. The low-baseline caffeine group had a treatment-bytime interaction, but post-hoc analysis revealed that there was only a significant difference from PLA before exercise.

Simple movement was also assessed by the executive function test. Main effects for both treatment and time were found, though there was no treatment-by-time interaction. Simple movement speed increased after exercise with both treatment drinks. While participants were faster on the day they received the ED, conclusions about ED's effects cannot be made due to a lack of interaction.

Gross movement time was also assessed. When assessed by the reaction time or the executive function test, participants demonstrated faster gross movement times after exercise as indicated by a main effect for time. A treatment effect was not seen as there were no interactions of treatment-by-time nor were main effects for treatment observed.

\section{Metabolic Effects}

\section{Plasma Substrates}

Treatment-by-time interactions were significant for insulin, blood glucose and free fatty acids. Main effects for treatment were also significant. Insulin was at fasting levels before receiving a treatment drink at both visits. After receiving ED, plasma insulin spiked, occurring concomitantly with a fall in free fatty acids while blood glucose 
remained unchanged. The exercise onset caused a drop in blood glucose when participants consumed ED, though glucose returned to a level that was not statistically different from PLA by $29 \mathrm{~km}$ into the race. Free fatty acids also increased as the exercise continued, and were not statistically different from PLA by $23 \mathrm{~km}$ into the race.

There was no treatment-by-time interaction for glycerol. Regardless of treatment drink provided, glycerol remained unchanged at rest then rose throughout the duration of exercise.

\section{CafFeine, CATECholamines, B-ENDORPhins ANd CoRTisol}

Treatment-by-time interactions were significant for caffeine, epinephrine and norepinephrine. Main effects for treatment were also found. ED elevated plasma caffeine levels throughout the race. At rest, neither catecholamine tested was affected by ED. Epinephrine was elevated due to ED at $6 \mathrm{~km}$ and remained above PLA until the end of the race. Norepinephrine was only different between ED and PLA at $6 \mathrm{~km}$.

No treatment-by-time interaction was found for $\beta$-endorphins; however, there was a main effect for time. Plasma concentrations fell from baseline values when participants were resting, and rose due to exercise. There was no difference at rest or at the conclusion of exercise between the treatments.

There was a main effect of time for cortisol. Participants had elevated levels of cortisol at the end of the exercise regardless of treatment.

\section{PHYSIOLOGICAL INDICATORS OF WORK OUTPUT}

A treatment-by-time interaction was found for lactate. A main effect for treatment was also found. The carbohydrates ingested in ED generated lactate in participants at rest. Throughout the exercise ED maintained lactate concentrations greater than those seen in PLA. 
Plasma myoglobin was measured at rest and throughout the exercise trials. There was a main effect for time, though no at no time was there a significant change from baseline as indicated by post-hoc testing.

Main effects for treatment and time were found for $\mathrm{VO}_{2}$ measured throughout the exercise trials. The RER had a main effect by time but was not affected by the treatment. Over the course of the race, oxygen consumption fell, though when participants consumed ED they sustained an overall greater $\mathrm{VO}_{2}$. RER fell over the course of the race also, though no main effect for treatment was observed.

Although there was no treatment-by-time interaction, there were main effects for treatment and time for HR. Throughout the ride ED caused an overall greater HR than PLA. 


\section{DISCUSSION}

The current study evaluated the effects of ED on physical, cognitive and psychomotor performance, and metabolism at rest and during exercise. The primary finding was that the ED enhanced aerobic exercise performance. Every participant completed the cycling time-trial faster when they had ED compared to PLA. The average time to finish was improved by more than 3\%. Furthermore, there was no difference in RPE between trials despite the fact that when participants received ED they had an increased exertion. The results clearly indicate that ED when provided prior to exercise enhances performance of trained cyclists. The enhanced performance is likely due to a combination of increased central drive and sympathetic output from caffeine acting as an adenosine antagonist in conjunction with readily available carbohydrate for rapid oxidation. However, it cannot be ruled out that the micronutrient composition of ED had a positive influence on exercise performance.

The current findings are in agreement with other research that has evaluated the effects of energy drinks on exercise performance. Ivy et al. (2009) found an improvement in cycling time-trial performance using Red Bull energy drink. Ivy et al. (2009) used a similar time-trial protocol to the current study and also provided the energy drink 40 minutes before the time trial, so it is not surprising that our results are similar. Alford et al. (2001) demonstrated that consuming Red Bull energy drink resulted in enhanced ability to sustain work output at a an exercise intensity that elicited $65-75 \%$ of $\mathrm{HR}_{\mathrm{MAX}}$. It has also been shown that consumption during exercise can be of benefit. In the study by Gei $\beta$ et al. (1994) participants had improved performance when provided Red Bull energy drink 30 minutes into an exercise bout at $70 \%$ of $\mathrm{VO}_{2 \mathrm{MAX}}$ lasting 1 hour, followed by an exercise test of increasing intensity to volitional fatigue. Similar to these previous 
studies, the current study compared an energy drink containing both caffeine and carbohydrates to a non-caloric caffeine-free placebo drink.

The effect of caffeine and carbohydrate combined on exercise performance has been studied previously. Kovacs et al., (1998) demonstrated that caffeine added to carbohydrates improved cycling time trial performance greater than carbohydrates alone. In the study, participants consumed an average of $1026 \mathrm{ml}$ of a placebo or 1 of 4 treatment drinks containing a total of 71 grams of carbohydrate and $0,154,230$, or 328 $\mathrm{mg}$ of caffeine. The treatments were standardized so that participants drank a total of 14 $\mathrm{ml} / \mathrm{kg}$ with $68.8 \mathrm{~g} / \mathrm{l}$ of carbohydrate and $0,150,225$, or $325 \mathrm{mg} / \mathrm{l}$ of caffeine. The doses were divided such that $57 \%$ of the total volume was ingested 55 minutes prior to a 1 -hour time-trial ride while the rest of the drink was consumed during the exercise bout. The researchers found that the moderate and high doses of caffeine improved performance on the time-trial ride greater than the carbohydrate alone, while the consumption of any of the treatment drinks improved performance compared to placebo. There were no statistically significant differences in performance between the carbohydrate-only and low-caffeine supplement.

The research by Kovacs (1998) indicates that the addition of at least $225 \mathrm{mg}$ of caffeine to a solution of 71 grams of carbohydrate enhances performance greater than the ingestion of carbohydrates alone. When the caffeine concentration was below $225 \mathrm{mg}$ it had no additive effect with carbohydrate on performance. By contrast ED in the current study provided participants with lower doses of both ingredients (54 g of carbohydrate and $160 \mathrm{mg}$ of caffeine). However, since we did not use a carbohydrate-only control treatment as in the research by Kovacs (1998), we cannot rule out the possibility that the improvement in performance was entirely due to carbohydrates or additional micronutrients found in the ED. In this regard, even the 5 participants that arrived to the 
lab with elevated blood caffeine levels improved time-trial performance by an average of $3 \%$ when they received ED. Notably the magnitude of increase in blood caffeine was still significant in participants with high initial blood concentrations, though the absolute increase was reduced (379 to 2324 and 1842 to $3196 \mathrm{ng} / \mathrm{ml}$ for low-baseline and highbaseline participants, respectively). It is therefore unlikely that caffeine is the only ingredient responsible for the ergogenic effect of the ED since participants with elevated baseline caffeine levels improved performance.

While we did not study ED against a carbohydrate-only control drink, there is evidence that caffeine in the ED was in part responsible for the ergogenic effect. Caffeine alone has been shown to lead to an uncoupling of exertion and perceived exertion. In an exercise protocol requiring participants to perform as much work as possible during 2 hours of cycling, those who ingested caffeine had enhanced work performance with no difference in RPE (Ivy et al., 1979). Similarly, caffeine enhanced performance on an 8$\mathrm{km}$ running race without increasing RPE (Bridge \& Jones, 2006). There are several possible explanations for the improved RPE induced by caffeine. Caffeine is known to increase central neuronal excitability, reduce the threshold of neuron activation (Waldeck, 1973) as well as potentiate muscle contractile force directly (Tarnopolsky \& Cupido, 2000). Caffeine has been found to act as an adenosine receptor antagonist (Fredholm, Chen, Masino, \& Vaugeois, 2005) and adenosine agonists have specifically been shown to induce fatigue in rats (Davis et al., 2003). It also has been shown to increase circulating $\beta$-endorphin levels during exercise (Laurent, 2000). In the current study we did not see a significant effect of ED on $\beta$-endorphins, but we did observe improved exercise performance without a consequent increase in RPE. Therefore, we propose that the uncoupling of RPE and true exertion we observed was the result of the 
action of caffeine on the central nervous system, and its direct potentiating effect on muscle force production.

Carbohydrates consumed within an hour of exercise onset have not always resulted in ergogenic effects (Foster et al., 1979), whereas caffeine consumption in this timeframe has consistently yielded exercise performance benefits (Graham \& Spriet, 1991; Ivy et al., 1979; Schneiker et al., 2006). The proposed mechanism by which carbohydrate feedings immediately before exercise may hinder performance is that an increase in insulin and blood glucose will down-regulate lipid oxidation and result in earlier depletion of carbohydrate energy stores (Foster et al., 1979). In the current study, we observed an increase in resting insulin concentrations and a subsequent fall in blood glucose at exercise onset, but only when participants received ED. The observed increase in $\mathrm{VO}_{2}$ without a significant shift in RER in the current study indicates that after consuming ED participants were metabolizing both carbohydrates and lipids at a faster rate during exercise without shifting the relative proportions: the lack of change in RER is in spite of the significant increase in catecholamines that we observed in this study. Caffeine has been shown to increase catecholamine levels in other exercise protocols (T.E. Graham \& Spriet, 1991; T.E. Graham et al., 2000; Spriet et al., 1992). The addition of caffeine to a carbohydrate supplement taken shortly before exercise onset may counter any inhibitory effects of hyperglycemia and hyperinsulinemia by activating intramuscular triglyceride oxidation and sparing muscle glycogen (Essig et al., 1980). Research using muscle biopsies to detect changes in muscle triglyceride and glycogen concentration as well as tracers to determine rates of uptake of blood glucose and FFA are necessary to specifically determine the exact sources of energy used during exercise when athletes consume a complex energy drink. 
In the current study we also examined the effects of ED on decision making and psychomotor performance before, during, and after exercise. Participants completed several tasks including a tapping test, a reaction time test, an executive function test (including aspects of both the tapping test and reaction time test), and a Stroop test. We demonstrated that consumption of ED will cause enhanced simple movement time as assessed by the tap test before and after intense exercise. The effect was not found in the executive function test. The heightened difficulty of the executive function test compared to the tap test is likely responsible for this discrepancy. During the executive function test participants tapped a button as fast as possible with 1 hand while the other hand was responsible for quickly moving to different locations based on a visual cue. Since participants garnered a benefit from ED on the tap test, but not on the same performance measure (taps per second) during the executive function test, we believe that simple psychomotor performance was aided by the ED but this beneficial effect is diminished during more complex bimanual tasks. The effect of the ED on tapping speed seems to be due to the caffeine content, since follow-up analysis revealed that only the participants who had low-baseline caffeine concentrations had significantly improved speed.

The ED did not result in a change in decision making ability as assessed by the executive function or Stroop tests. Both caffeine and carbohydrate in isolation have beneficial effects on cognitive performance. Taken 40 minutes prior to a task, caffeine ingestion has improved movement time in volunteers (Durlach, 1998), while carbohydrate ingestion has been found to benefit memory (Benton \& Owens, 1993). In the present study memory was not evaluated, so the effect of ED on this aspect of cognitive function cannot be assessed. A limitation of this work is that a ceiling effect was seen for both methods of evaluating decision making ability where most participants 
completed the tasks with $100 \%$ accuracy. The consequences of the ceiling effect are compounded by the small sample size, and a type II error may have been made.

The ED resulted in less precision during the tap test, though the loss of accuracy was not ecologically significant. ED did not result in differences on other cognitive measures. Since ED enhanced simple movement time without resulting in a clinically significant loss in accuracy, it may be beneficial for athletes to ingest prior to exercise to enhance psychomotor performance.

A main effect for time was observed for reaction time indicating that regardless of treatment drink provided, exercise enhanced this aspect of psychomotor performance. Working extensively with soccer players, McMorris demonstrated that exercise itself enhances movement time. In a series of studies, soccer players had faster decisionmaking time when exercising at high intensities on a cycle ergometer (McMorris, 1997), and that this improvement in decision-making time was paired with elevated levels of epinephrine (McMorris, 1999). The participants in the current study showed rising epinephrine levels as the time of exercise increased and subsequently had faster performance on the Stroop test. However, despite significant differences in plasma epinephrine levels between ED and PLA there were no differences in Stroop scores.

Since this study demonstrated ergogenic properties of ED using a smaller caffeine dose than that which has been traditionally studied (Costill, Dalsky, \& Fink, 1978; Graham \& Spriet, 1995; Hulston \& Jeukendrup, 2008; Ivy et al., 1979), we believe that further study into the synergistic effects of the ingredients is warranted. Specifically, utilizing alternative treatment drinks (carbohydrate-only, caffeine-only, micronutrientonly) in addition to complete ED and PLA treatments in a protocol similar to ours will lend further insight into which ingredients are responsible for the ergogenic effects. 
Additionally, dose-response studies will be useful to elucidate what the optimal ratio of the active ingredients is for athletic performance.

Studies that examine the effectiveness of the product on longer and shorter duration events are also justified since caffeine has been shown to benefit exercisers in a 2000-meter rowing event lasting approximately 6 minutes, in 1 study (Bruce et al., 2000), but not another (Skinner et al., 2010). Furthermore, ingestion of caffeine alone improved running performance over 30-40 minutes in an $8-\mathrm{km}$ race (Bridge \& Jones, 2006). The complex formula of ED may benefit athletes participating in events of shorter duration than we tested in this study.

While the methods used to assess cognitive performance in the current study were chosen for ease of implementation and data acquisition, they did not provide ecological validity: the manner in which we evaluated reaction time required the movement of relatively small muscle groups (the fingers and forearms only), whereas whole-body reaction time is more relevant for most athletic events. In this regard, a positive effect of caffeine on whole-body reaction time has been demonstrated (Duvnjak-Zaknich, Dawson, Wallman, \& Henry, 2011). The researchers found that caffeine in isolation enhanced the abilities of team-sports athletes to react to sports-specific stimuli before and after a simulated game. Future research may find an effect of ED on reaction time if it is assessed during movements that more mimic movement associated with various sports.

A final set of future studies that can be developed from the current work involves examining the metabolic effects of ED in non-exercising conditions. Caffeine may alter insulin sensitivity, so tests of ED's effects on glucose tolerance in healthy and insulin insensitive individuals are warranted. Furthermore, caffeine has been demonstrated to elevate resting metabolic rate in both athletes and non-athletes, so investigation into this product's ability to alter basal metabolism is justified. 
In conclusion, the ED resulted in improved cycling time-trial performance as well as improved performance on a simple psychomotor test. Participants who arrived to the laboratory with elevated basal caffeine levels benefitted on the time-trial race to the same extent as those who arrived with low basal caffeine levels, implying that the combination of caffeine and carbohydrates in the ED were responsible for the ergogenic effects. Only participants with low-baseline caffeine levels had improved tapping speeds due to ED, suggesting that caffeine specifically was the responsible ingredient for improving this aspect of psychomotor performance. However, the addition of other micronutrients may have also influenced our outcomes. Follow-up work utilizing carbohydrate-only and caffeine-only controls will shed light on the relative contributions and interactions of these ingredients in improving athletic performance. 


\section{LITERATURE REVIEW \\ Introduction}

Pre-exercise nutritional supplementation is commonly practiced among endurance athletes in order to enhance physical and cognitive performance. Red Bull energy drinks when ingested one hour before a cycling time-trial ride lasting approximately 60 minutes were shown to improve race times by $3 \%$ (Ivy et al., 2009). In addition to enhancing athletic performance, energy drink consumption has been shown to improve reaction time and reduce reports of mental fatigue in a study of 80 adults aged 18-40 (Howard \& Marczinski, 2010).

There are a multitude of ingredients in energy drinks including caffeine, carbohydrate, carnitine, ginseng, glucuronolactone, grape seed extract, guaraná, inositol, milk thistle, and taurine (Babu, Church, \& Lewander, 2008). The sugar content of energy drinks ranges from 0-47 grams per 8 oz. serving while the caffeine content ranges from 24-640 mg (Heckman, Sherry, \& Gonzalez de Mejia, 2010). For comparison, 8 oz. of unsweetened American coffee contains 76$131 \mathrm{mg}$ of caffeine and 0 grams of sugar (Santini et al., 2011).

This literature review will systematically report the research related to exercise and cognitive performance effects of energy drink consumption. Aerobic exercise performance effects of the following energy drink ingredients will be analyzed: caffeine, carbohydrate, ginseng, glucuronolactone, guaraná, inositol, and taurine. The same ingredients will then be

discussed in regards to their effects on cognitive performance. Since exercise itself impacts cognitive performance (McMorris, Sproule, Turner, \& Hale, 2011), the interaction between exercise and cognition in the absence of nutritional supplementation will be examined next. Finally a conclusion about the effects of an energy drink on aerobic and cognitive performance during and after exercise will be made. 


\section{The Effects of Energy Drink Ingredients on Aerobic Performance}

\section{Caffeine Enhances Aerobic Exercise Performance}

Caffeine has been studied for ergogenic potential since the early $20^{\text {th }}$ century. Researchers quantified the amount of work performed in successive sets of resistance exercise on an ergograph (see Figure 1) by 2 individuals when they had received either caffeine or a placebo (Rivers \& Webber, 1907). Other early work demonstrated that caffeine enhanced handgrip strength and endurance (Thornton et al., 1939) and restored work capacity after fatigue (Alles \& Feigen, 1942) better than a placebo. These studies gave participants doses in the range of 1.4 to $5.7 \mathrm{mg} / \mathrm{kg}$. While the results were encouraging, there was a lack of statistical analysis to objectively evaluate the data.

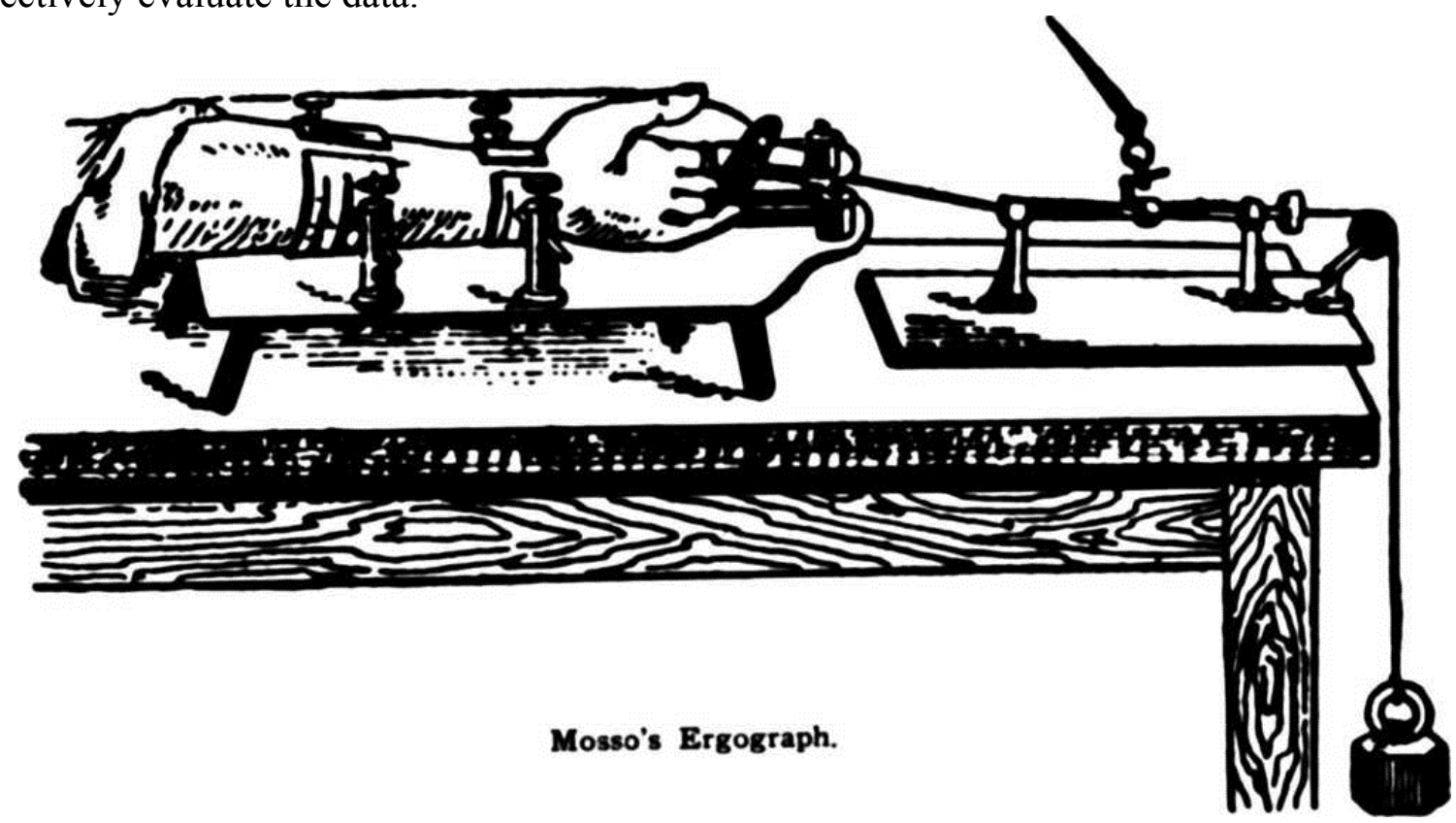

Figure 1 An ergograph. A participant lifts a weight via a pully. The lifting motion causes deflection of a pen which will trace a line with a length that is directly related to the amount of work performed. Work was quantified by this method in early research (Alles \& Feigen, 1942; Rivers \& Webber, 1907).

In the late 1970 s and early 1980 s there was resurgence in research on caffeine as a supplement for athletes. If given caffeinated coffee (providing $330 \mathrm{mg}$ of caffeine) 60 minutes before exercising, cyclists rode 15 minutes longer at $80 \%$ of $\mathrm{VO}_{2 \max }$ than if they were given decaffeinated coffee (Costill et al., 1978). If given decaffeinated coffee, the athletes in the study had higher ratings of perceived exertion (RPE) throughout the ride though there was no 
difference in RPE at the time of fatigue. The RER was lower when subjects had consumed caffeine. FFA and glycerol were greatest when participants received caffeine at all times measured, though no statistically significant pairwise comparisons were reported.

Caffeine in isolation has enhanced aerobic performance in a variety of exercise conditions. Over the course of a 2-hour cycling bout, exercisers performed more work and maintained a higher average $\mathrm{VO}_{2}$ compared to placebo without an increase in RPE if they were provided with $500 \mathrm{mg}$ of caffeine in divided doses (Ivy et al., 1979). The benefits of taking caffeine are not limited to cycling performance since when given $9 \mathrm{mg} / \mathrm{kg}$ of caffeine 1 hour prior to exercise athletes had prolonged endurance at $85 \%$ of $\mathrm{VO}_{2 \max }$ whether tested on a treadmill or cycle ergometer (Graham \& Spriet, 1991). Even in lower doses caffeine prolonged running time to exhaustion at $85 \%$ of $\mathrm{VO}_{2 \max }$ better than a placebo if provided as $3-9 \mathrm{mg} / \mathrm{kg} 1$ hour prior to exercise onset (Graham \& Spriet, 1995). Caffeine is ergogenic even in athletic events that are of shorter duration since runners performed an $8-\mathrm{km}$ race $1.2 \%$ faster if they had 6 $\mathrm{mg} / \mathrm{kg}$ caffeine 1 hour before (Bridge \& Jones, 2006).

The ergogenic effects of caffeine are not limited to prolonged endurance activity as several studies have also shown benefits in exercise protocols that include a significant glycolytic component (Bruce et al., 2000; Schneiker et al., 2006; Wiles et al., 1992). Athletes completed a $1500-\mathrm{m}$ race faster and with an increased $\mathrm{VO}_{2}$ and blood lactate concentration if they were provided with caffeine before the race (Wiles et al., 1992). Rowers finished a 2000-m race faster when they were provided $6 \mathrm{mg}$ caffeine per $\mathrm{kg}$ body mass (Bruce et al., 2000). However in a study by Skinner (2010) this fining could not be replicated. Team sports athletes produced more power during intermittent sprints in both halves of a 72-minute exercise protocol when they had ingested caffeine before competition (Schneiker, Bishop, Dawson, \& Hackett, 2006b). Cyclists have been shown to complete more work in a 60-second cycling time-trial if they received $5 \mathrm{mg} / \mathrm{kg}$ of caffeine 1 hour before the time-trial (Wiles et al., 2006).

Caffeine has ergogenic effects on aerobic performance whether the athlete is a habitual consumer of caffeine or not. Both habitual and non-habitual users of caffeine had improved 
times to exhaustion at $80 \%$ of $\mathrm{VO}_{2 \max }$ if given $5 \mathrm{mg} / \mathrm{kg}$ of caffeine $1-3$ hours prior to exercise onset (Bell \& McLellan, 2002). Two studies which were already discussed also showed that caffeine had ergogenic effects whether participants were regular consumers or not (T.E. Graham \& Spriet, 1991; J.D. Wiles et al., 1992).

It had been demonstrated that caffeine increased the blood FFA level (Bellet, Kershbaum, \& Finck, 1968), and that a non-caffeine induced increase in circulating FFA led to subsequent increases in endurance performance (Costill et al., 1977). The observation that RER was lower and plasma FFA were higher during exercise following consumption of caffeine (Costill et al., 1978; Ivy et al., 1979) led researchers to speculate that caffeine was ergogenic due to enhanced usage of lipids and a sparing of muscle glycogen. This was partially supported by Spriet et al. (1992) who found that ingestion of $9 \mathrm{mg} / \mathrm{kg}$ of caffeine 1 hour prior to exercise prolonged time to exhaustion at $80 \%$ of $\mathrm{VO}_{2 \max }$ and spared muscle glycogen. Doses as low as $5 \mathrm{mg} / \mathrm{kg}$ have also been shown to reduce muscle glycogen depletion during moderate intensity exercise (Erickson et al., 1987; Essig et al., 1980). 


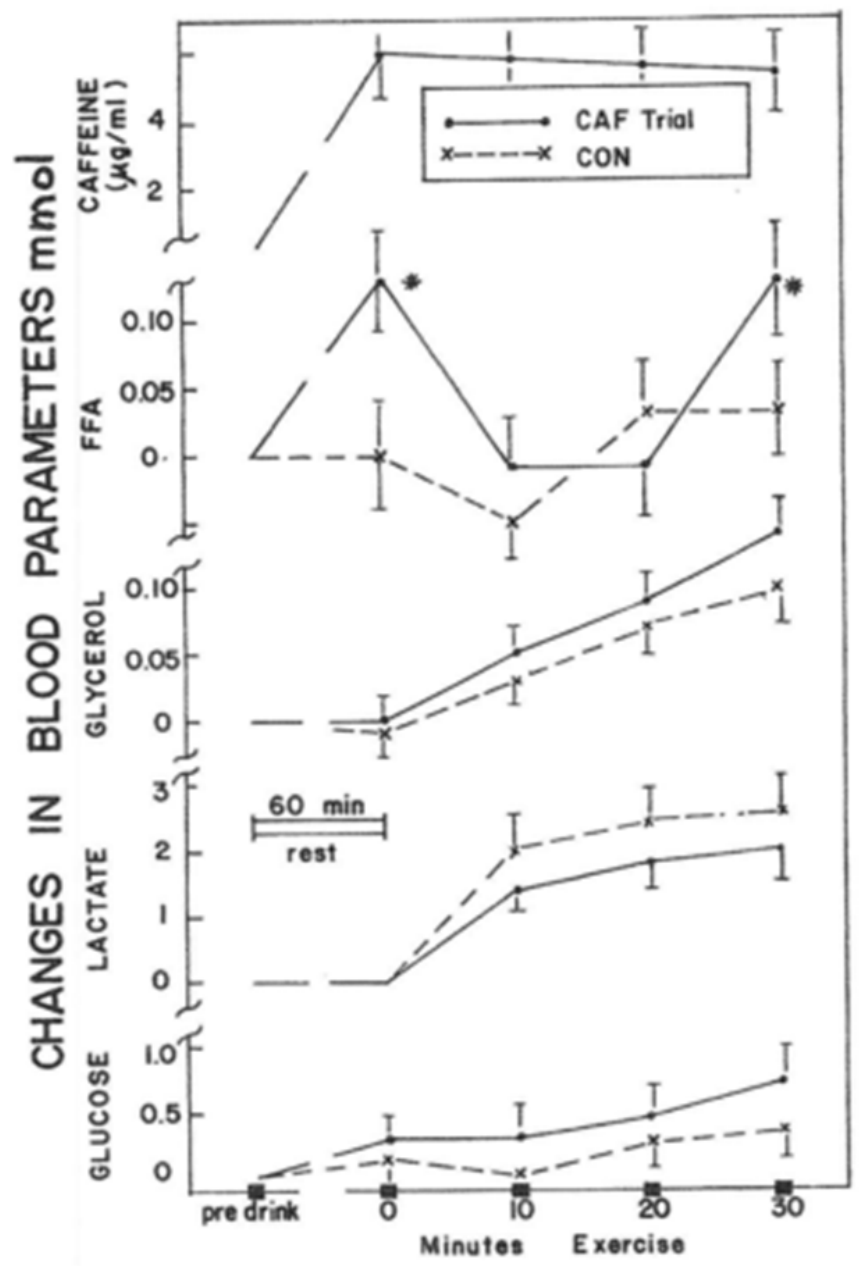

Figure 2 From Essig et al. (1980). Caffeine elevated resting blood FFA and by 30 minutes of exercise of moderate intensity.

The support for the metabolic hypothesis is not unequivocal. Costill's group reported differences in RER, plasma FFA, and blood glycerol during exercise performance after participants received caffeine (Costill et al., 1978; Essig et al., 1980; Ivy et al., 1979), but the differences were only statistically significant in two of the studies (Essig et al., 1980; Ivy et al., 1979) (see Figure 2). The glycogen sparing effect of caffeine was paired with a lower RER and increase in FFA in the studies by Essig et al. (1980) and Ivy et al. (1979), but not in two other studies (Erickson et al., 1987; Spriet et al., 1992). Casal and Leon (1985) found that caffeinated coffee increased plasma FFA at rest but did not result in differences in $\mathrm{VO}_{2}$, or RER during 45 minutes of running at $75 \%$ of $\mathrm{VO}_{2 \max }$ compared to decaffeinated coffee or control, nor was there a treatment effect on post-exercise measures of blood FFA or glucose. If participants followed a 
3-day carbohydrate loading protocol, there were no metabolic shifts (as indicated by blood FFA, blood glucose, $\mathrm{VO}_{2}$ and RER) due to $6.5 \mathrm{mg} / \mathrm{kg}$ of caffeine provided 3 hours before a 2-hour cycling bout at $75 \% \mathrm{VO}_{2 \max }$ (Weir et al., 1987). Utilizing a combination of arterial and venous blood samples and muscle biopsies from men exercising for 1 hour at $70 \%$ of $\mathrm{VO}_{2 \max }$, Graham et al. (2000) found that ingesting $6 \mathrm{mg} / \mathrm{kg}$ of caffeine had no effect on substrate utilization at the active muscle. However, the lipolytic effect of caffeine may require exercise to be in excess of 60 minutes (Ivy et al., 1979).

When caffeine and carbohydrate are supplemented together, exercise performance is enhanced, but lowered plasma FFA indicate the ergogenic effect is not entirely due to caffeineinduced lipolysis (Ivy et al., 2009; Kovacs et al., 1998). Caffeine in the amount of 154-328 mg added to a $70 \mathrm{~g}$ carbohydrate and electrolyte solution (including $72 \mathrm{mg}$ taurine) enhanced exercise performance greater than the carbohydrate electrolyte solution alone though plasma FFA were lower than when participants received a placebo (Kovacs et al., 1998) (see Figure 3). The exercise protocol used was a time-trial in which participants completed a set workload that was equivalent to exercising at $70 \%$ of $\mathrm{VO}_{2 \max }$ at $90 \mathrm{rpm}$ for one hour. Using the same exercise protocol, Red Bull energy drink (consisting of $160 \mathrm{mg}$ of caffeine, $54 \mathrm{~g}$ of carbohydrate, $2 \mathrm{mg}$ of taurine, and $1.2 \mathrm{~g}$ glucuronolactone) shortened the time to finish by $3 \%$ but FFA and glycerol were reduced (Ivy et al., 2009). Moreover, Hulston and Jeukendrup (2008) demonstrated no difference in RER, or rate of appearance, or disappearance of blood glucose between treatments when participants completed a time trial while consuming a $6.4 \%$ carbohydrate solution or the same solution with $5.3 \mathrm{mg} / \mathrm{kg}$ body mass of caffeine added. 


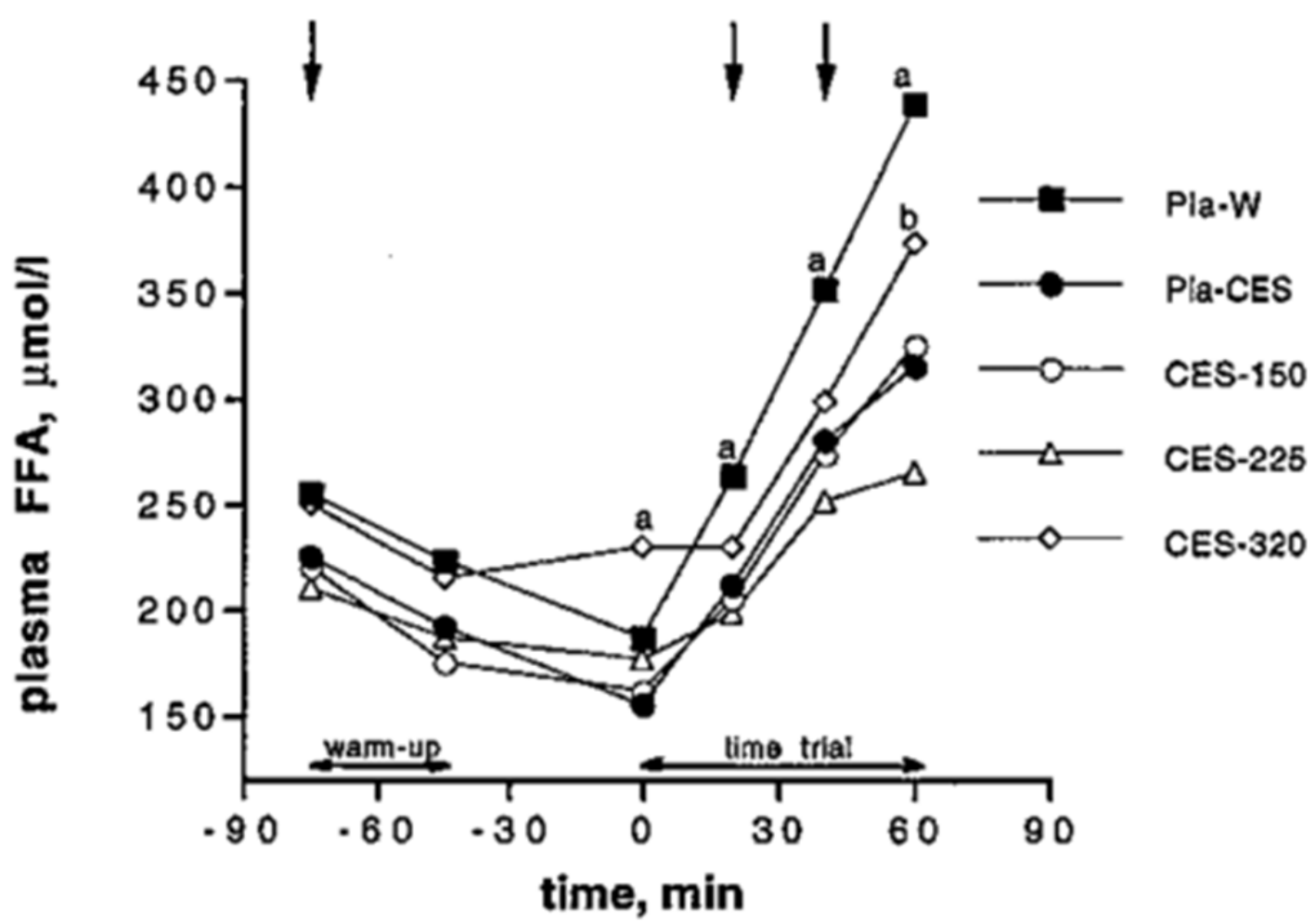

Figure 3 From Kovacs et al., (1998). CES=carbohydrate electrolyte solution. 150, 225, 320= approximate dose in mg of caffeine delivered in total.

The exact physiological mechanism responsible for caffeine's ability to enhance exercise performance is elusive. Caffeine may act ergogenically due to actions on the sympathetic nervous system (SNS) via the central nervous system (Graham et al., 2000). Epinephrine increases in the blood at rest and during exercise following $6 \mathrm{mg} / \mathrm{kg}$ of caffeine consumption (T.E. Graham \& Spriet, 1991, 1995; Graham et al., 2000; Spriet et al., 1992). Red Bull energy drink also elevated epinephrine (Ivy et al., 2009). Additionally, RPE has remained unchanged from placebo though exercise performance has actually increased in several studies (Bridge \& Jones, 2006; Costill et al., 1978; J.L. Ivy et al., 1979; J.D. Wiles et al., 1992) indicating that caffeine may have blunted central fatigue. This line of thought is supported by work in rodent models where it has been shown that caffeine's primary physiological function in the central nervous system is as an adenosine antagonist, specifically binding $A_{1}$ and $A_{2 A}$ receptors which normally inhibit wakefulness and motor activity, respectively (Fredholm et al., 2005). 
Furthermore, caffeine may reduce central fatigue by inducing the release of endogenous painkillers, $\beta$-endorphins. Laurent (2000) demonstrated that ingestion of $6 \mathrm{mg} / \mathrm{kg}$ of caffeine 90 minutes before exercise led to increased plasma $\beta$-endorphins compared to placebo. The study by Ivy et al. (2009) described previously showed a tendency for $\beta$-endorphins to be elevated by the end of exercise when subjects were administered caffeine compared to placebo, though the difference was not statistically significant $(\mathrm{p}=0.10)$.

A final explanation for caffeine's ability to induce ergogenesis is modification of calcium handling within the sarcoplasm resulting in lowered threshold for excitation-contraction coupling. Specifically it has been shown that muscles in humans will contract with more force at submaximal stimulation frequencies after a dose of $500 \mathrm{mg}$ of caffeine (Lopes et al., 1983). Doses of $6 \mathrm{mg} / \mathrm{kg}$ administered to habituated or non-habituated caffeine consumers also induced increased force production at submaximal frequency stimulations, though maximal voluntary contractions were unaltered (Tarnopolsky \& Cupido, 2000).

Caffeine has repeatedly been shown to enhance exercise performance in events requiring a significant amount of energy production from aerobic metabolism (Graham \& Spriet, 1991; Ivy et al., 1979; Schneiker et al., 2006). The effects are mostly likely mediated through a combination of metabolic action (Costill et al., 1978), stimulation of the central nervous system and up-regulation of the SNS (Graham et al., 2000), or modification of intramuscular calcium handling resulting in a reduced threshold for neuromuscular action (Tarnopolsky \& Cupido, 2000).

\section{CARbOHYDRATE TYPE AND TIMING INFLUENCE EFFECTIVENESS OF CARBOHYDRATE AS AN ERGOGENIC AID}

\section{Carbohydrate Fed During Exercise Enhances Performance}

Carbohydrate feedings of 120 grams of glucose polymer over the course of 2.5 hours of walking exercise ( $45 \%$ of $\mathrm{VO}_{2 \max }$ ) prolonged endurance by $11.5 \%$ (Ivy et al., 1983). Similarly, when athletes were fed carbohydrate during exercise at $70 \%$ of $\mathrm{VO}_{2 \max }$ they had an elevated RER 
and prolonged endurance without altering the rate at which muscle glycogen was utilized (Coyle et al., 1986). Another study showed that when athletes were fed carbohydrate during prolonged intermittent intensity exercise ranging between $45-75 \%$ of $\mathrm{VO}_{2 \max }$, endurance was improved and this was in part due to a sparing of muscle glycogen (Yaspelkis et al., 1993).

More recently it was shown that adding amino acids or caffeine to a carbohydrate supplement fed during exercise enhanced performance greater than if fed carbohydrate alone (Hulston \& Jeukendrup, 2008; Ivy et al., 2003). Cyclists rode on ergometers at varying intensities between $45-75 \%$ of $\mathrm{VO}_{2 \max }$ for 3 hours before completing a ride to exhaustion at $85 \%$ of $\mathrm{VO}_{2 \max }$ (Ivy et al., 2003). Athletes fed a 7.75\% carbohydrate solution rode 7 minutes (55\%) longer than if they received placebo, and the addition of $1.94 \%$ protein to the solution further increased ride time to exhaustion by another 7.2 minutes $(56 \%)$. When participants consumed a drink containing $5.3 \mathrm{mg} / \mathrm{kg}$ body mass of caffeine in addition to $6.4 \%$ carbohydrate, they finished a time-trial race faster than if they consumed the carbohydrate alone (Hulston \& Jeukendrup, 2008). Therefore, carbohydrate supplementation is very capable of working additively with other forms of ergogenic aids.

\section{Carbohydrate Fed Before Exercise Has Equivocal Effects on Aerobic Performance}

While carbohydrate feedings during exercise aid endurance performance, carbohydrate feedings before exercise have been demonstrated to inhibit (Foster et al., 1979), enhance (Kirwan et al., 1998; William M. Sherman et al., 1989), or have no effect on (Hargreaves et al., 1987; Kirwan et al., 1998) exercise performance depending on the type of carbohydrate provided and timing of ingestion. Participants benefitted from carbohydrate feeding when it was provided 4 hours prior to exercise initiation (Sherman et al., 1989) or if provided within an hour of exercise when it had a low glycemic impact (Kirwan et al., 1998). High glycemic impact carbohydrate feedings in the hour leading up to exercise have produced either no effect on exercise

performance (Hargreaves et al., 1987; Kirwan et al., 1998; Kovacs et al., 1998) or have resulted in earlier fatigue (Foster et al., 1979). Recently it has been demonstrated that when athletes are 
fed a complete meal 3 hours prior to exercise, the ingestion of $2.5 \mathrm{~g} / \mathrm{kg}$ body mass of carbohydrate in the hour before exercise had no effect on performance (Rollo \& Williams, 2010).

The consumption of carbohydrate in the hour leading up to exercise has been associated with a rebound hypoglycemia early in exercise due to the dual effects of insulin- and contractionstimulated glucose clearance (Foster et al., 1979). While hypoglycemia was noted in several studies when carbohydrate was ingested immediately prior to exercise (Foster et al., 1979; Hargreaves et al., 1987; Kirwan et al., 1998), blood sugar did not always fall under these circumstances (Rollo \& Williams, 2010), and exercise performance was not always negatively impacted (Hargreaves et al., 1987; Kirwan et al., 1998; Kovacs et al., 1998). It is no longer recommended that athletes make an effort to avoid carbohydrate feedings before exercise unless it is known to cause them personal gastric upset (Jeukendrup \& Killer, 2010).

\section{Conclusion on the Effects of Carbohydrate on Aerobic Exercise Performance}

Carbohydrate fed to athletes during aerobic exercise consistently resulted in improved aerobic performance (Coyle et al., 1986; Ivy et al., 1983; Ivy et al., 2003; Yaspelkis et al., 1993) and the addition of protein (Ivy et al., 2003) or caffeine (Hulston \& Jeukendrup, 2008) enhanced performance further. While the early research indicated that consumption of carbohydrate in the hour leading into an exercise bout would cause a detriment in performance (Foster et al., 1979), if the carbohydrate source is in the form of a mixed meal it is not likely to hinder performance (Foster et al., 1979; Kirwan et al., 1998) and recent recommendations do not suggest avoiding carbohydrate before exercise (Jeukendrup \& Killer, 2010).

\section{OTHER ENERGY DRINK INGREDIENTS LACK SUFFICIENT RESEARCH TO CONCLUDE ERgogenic Properties}

\section{Ginseng}

Ginseng in isolation has not been evaluated as a possible ergogenic aid. One study has revealed that ginseng had differing effects on blood glucose at rest depending on if it was ingested with or without carbohydrate (Reay et al., 2006). At rest, doses of 200-400 mg of 
ginseng reduced blood sugar for at least 2 hours compared to placebo. When the $200 \mathrm{mg}$ of ginseng was combined with a $25 \mathrm{~g}$ bolus of glucose, blood sugar levels were no different than when the glucose bolus was ingested alone indicating that any hypoglycemic effects of ginseng are lost when it is consumed with carbohydrate. While it remains to be studied, the ingestion of ginseng alone may hinder aerobic performance by causing early depletion of blood sugar levels, though co-ingestion with carbohydrate may attenuate these effects.

\section{Glucuronolactone}

Glucuronolactone is an intermediate in the pentose phosphate pathway (PPP) that serves to generate NADPH, nucleic acids, and pentose sugars. Glucuronolactone is also generated endogenously and can be used by the liver to modify toxic substances through glucuronidation.

A study examining glucuronolactone's effects on exercise performance directly was conducted in rats (Tamura et al., 1968). The researchers gave the rats intraperitoneal (IP) injections of glucuronolactone or other intermediates of the PPP between bouts of exhaustive swimming. After a third bout of fatiguing swimming, the rats were sacrificed and the liver was tested for its ability to neutralize toxic substances. The rats given glucuronolactone had enhanced swimming times and the greatest amount of liver enzyme activity (o-aminophenylglucuronide synthesis and $\beta$-D-glucuronide glucuronohydrolase activity) compared to rats injected with other PPP intermediates or a saline control. Though these effects were promising, each IP injection was $100 \mathrm{mg} / \mathrm{kg}$ body mass, which is a larger amount than has been tested in humans via any route of delivery.

Another study demonstrated a positive effect on exercise performance when $1200 \mathrm{mg}$ glucuronolactone and $2000 \mathrm{mg}$ taurine were added to a caffeine $(160 \mathrm{mg}$ ) and carbohydrate (54 g) beverage given to athletes while completing moderate intensity cycling exercise (Gei $\beta$ et al., 1994). Athletes rode at $70 \%$ of $\mathrm{VO}_{2 \max }$ for 60 minutes prior to completing a ramp protocol where workload was increased by $50 \mathrm{~W}$ every 3 minutes until work output fell by $10 \%$. The treatment drinks were given 30 minutes into the submaximal ride and the drink containing all ingredients 
(Red Bull energy drink) resulted in improved performance during the ramp test compared to if the drink was lacking in glucuronolactone and taurine. A similar ramp protocol was performed by the athletes 24 hours later with similar results; if the athletes consumed a drink with all ingredients during the first exercise protocol they had enhanced endurance compared to when they consumed a drink that lacked glucuronolactone and taurine. Counter to other research (Kovacs et al., 1998), this study (Gei $\beta$ et al., 1994) also demonstrated that a drink containing only carbohydrate resulted in enhanced endurance performance relative to a drink containing both caffeine and carbohydrate.

Glucuronolactone has potential as an ergogenic aid but there is a lack of research examining the effects of this ingredient in isolation on human aerobic performance. Glucuronolactone may enhance exercise performance by preventing the buildup of toxic products of exercise through glucuronidation or by sparing liver glycogen from being diverted into the PPP for this purpose.

\section{Guaraná}

Guaraná is a seed extract that contains caffeine as well as other phytochemical components. Guaraná is primarily marketed as a thermogenic aid and as a weight-loss supplement. Guaraná has been studied as a thermogenic supplement but usually in combination with other ingredients (Bérubé-Parent et al., 2005; Haller et al., 2005; Sale et al., 2006) and rarely has it been studied in exercising humans (Sale et al., 2006).

In combination with green tea extracts, guaraná increased 24-hour energy expenditure (by more than 150 Calories) while also elevating blood pressure (by 7 and $5 \mathrm{mmHg}$ systolic and diastolic respectively) in healthy adults (Bérubé-Parent et al., 2005). These effects were likely due to the guaraná and not the green tea extract since several different doses of green tea were used with no difference in results. Unfortunately, the researchers did not report the exact amount of guaraná extract used, only that it was sufficient to produce $600 \mathrm{mg}$ of caffeine over 3 doses per day. In addition to blood pressure, another study revealed that the effects of $250 \mathrm{mg}$ of 
guaraná extract and $325 \mathrm{mg}$ of ephedra extract in overweight individuals at rest increased HR, postprandial glucose, postprandial insulin, and postprandial FFA (Haller et al., 2005). A third study examining the combined effects of guaraná, bitter orange, and green tea extract in a proprietary blend failed to demonstrate any metabolic shifts in obese men at rest or during moderate walking exercise at $60 \%$ of $\mathrm{VO}_{2 \max }$ (Sale et al., 2006). The dose of guaraná delivered in this study was not explicitly stated, though it was less than $1262 \mathrm{mg}$.

Guaraná has hypertension-inducing properties (Bérubé-Parent et al., 2005; Haller et al., 2005). Caffeine is known to cause elevations in blood pressure (James \& Gregg, 2004) and elevations in catecholamines (Papadelis et al., 2003), which could result in elevated blood pressure and HR. Since guaraná extract is always delivered in combination with caffeine, it is difficult to ascertain the unique contributions of guaraná to these physiological outcomes. Furthermore, the research in humans has always delivered guaraná with other dietary supplements (Bérubé-Parent et al., 2005; Haller et al., 2005; Sale et al., 2006), making conclusions about guaraná more convoluted. The ergogenic potential of guaraná may be limited to the ergogenic potential of caffeine.

\section{Inositol}

Inositol plays a role in various physiological functions. The focus of inositol supplementation research is as a therapeutic agent in various specialized populations including prenatal (Copp \& Greene, 2010), neonatal (Hallman et al., 1992), individuals suffering from psoriasis due to lithium medication (O'Brien \& Koo, 2006), and postmenopausal women with metabolic syndrome (Giordano et al., 2011). Apart from when inositol is an ingredient in energy drinks, there is a lack of research on the effects of inositol on athletic performance.

\section{Taurine}

Taurine is a nonessential amino acid that has been implicated in the proper functioning and maintenance of skeletal muscle (Pierno et al., 1998; Warskulat et al., 2004). Taurine may provide cardiovascular health benefits by reducing SNS activity: for a review see Abebe \& 
Mozaffari (2011). Elderly Wistar rats that ingested $1 \mathrm{~g} / \mathrm{kg}$ of taurine daily for 2-3 months had greater intramuscular concentrations and improved responsiveness to electrical stimulation than unsupplemented controls (Pierno et al., 1998). If the transporter for taurine (taurine transporter system, taut) is not present due to gene knockout, rats have a reduction in treadmill exercise capacity of $80 \%$ or more demonstrating that taurine is normally a critical player in exercise performance (Warskulat et al., 2004).

A study already highlighted in this review demonstrated an enhanced aerobic performance in athletes after consuming a Red Bull energy drink containing $2000 \mathrm{mg}$ of taurine and $1200 \mathrm{mg}$ of glucuronolactone compared to a similar drink lacking these two ingredients (Gei $\beta$ et al., 1994). The increase in exercise performance was also paired with significantly reduced blood concentrations of epinephrine and norepinephrine, which the author concluded were due to inhibitory action of taurine on the SNS. The conclusion is controversial since research on caffeine and exercise has routinely demonstrated an increase in epinephrine when performance is also enhanced (Graham \& Spriet, 1991; Graham et al., 2000; Spriet et al., 1992), so a down-regulation of epinephrine would not be expected to necessarily lead to improved endurance.

Unfortunately acute supplementation of taurine in humans has not been studied in isolation. At least two research papers cite a 1987 paper by M. Ono published in Sulfur Amino Acids titled "Effects of taurine on the metabolism under physical exercise" (Alford et al., 2001; Gei $\beta$ et al., 1994) but searches of Pubmed, Elsevier, and Googlescholar all failed to turn up a matching journal or article by this title. The studies on the effects of taurine on exercise performance have been performed in rats (Pierno et al., 1998; Warskulat et al., 2004) or when it is present with other ingredients (Gei $\beta$ et al., 1994). Taurine may act as an ergogenic aid, but the mechanism has not been sufficiently studied in humans. 


\section{Conclusion on the Effects of Other Ingredients on Aerobic Exercise Performance}

Aside from caffeine and carbohydrate, the ingredients often present in energy drinks do not have sufficient scientific evidence to support their use as ergogenic aids. Ginseng ingested without carbohydrate may be ergolytic if it induces hypoglycemia (Reay et al., 2006). Glucuronolactone delivered in high concentrations via IP injection revealed promising results in rats (Tamura et al., 1968), but follow-up research in humans is lacking. Guaraná has demonstrated metabolic effects that suggest an up-regulation of SNSA (Haller et al., 2005), which may translate into enhanced exercise performance comparable to what is seen with caffeine (Graham, 2001), but the studies are flawed in that guaraná is always delivered with caffeine and other ingredients. Inositol's effects on exercise performance have not been tested. Taurine is a necessary component in young exercising muscle (Warskulat et al., 2004), but it has yet to be demonstrated that taurine supplementation will enhance exercise performance in humans.

\section{Energy Drinks Reliably Enhance Aerobic Performance}

Compared to ingesting a placebo, participants that ingested a consumer-size serving of Red Bull energy drink (80 mg caffeine, 27 g carbohydrate, $600 \mathrm{mg}$ glucuronolactone, $50 \mathrm{mg}$ inositol, $1000 \mathrm{mg}$ taurine) were able to exercise at a moderate workload (HR maintained between $65-75 \%$ of $\mathrm{HR}_{\max }$ ) for a longer period of time (Alford et al., 2001). Other work used twice as much Red Bull but also demonstrated enhanced aerobic performance with a modest dose of caffeine (160 mg) compared to placebo (Ivy et al., 2009). Red Bull was also demonstrated to prolong endurance on a ramp protocol after participants completed 60 minutes of exercise at $70 \%$ of $\mathrm{VO}_{2 \max }(\mathrm{Gei} \beta$ et al., 1994).

Traditionally, ergogenic doses of caffeine were at least $3 \mathrm{mg} / \mathrm{kg}$ of body mass (Costill et al., 1978; Graham \& Spriet, 1995; Ivy et al., 1979; Schneiker et al., 2006). When combined with carbohydrate and taurine only (Kovacs et al., 1998) or as part of a more complex energy drink (Alford et al., 2001; Ivy et al., 2009) doses of only 80-160 mg have been effective. Conversely, 
an energy patch delivering $74 \mathrm{mg}$ of caffeine transdermally did not enhance performance compared to a placebo patch on repeated Wingate tests (Dragoo et al., 2011) indicating that the low dose of caffeine is not effective in the absence of carbohydrate.

A proprietary blend of caffeine, glucuronolactone, and taurine as well as $40 \mathrm{kcal}$ from $8 \mathrm{~g}$ of amino acids and sucralose was also demonstrated to enhance aerobic exercise performance as assessed by time to fatigue (Walsh et al., 2010). The total amount of caffeine, glucuronolactone and taurine in the powder (called Amino Impact) was $2.05 \mathrm{~g}$ though the authors did not specify the exact proportions of each component. Participants in the study ingested a serving of the energy drink powder dissolved in $500 \mathrm{ml}$ of water 10 minutes prior to initiating exercise at $70 \%$ of $\mathrm{VO}_{2 \max }$. Though the supplement ingestion was placed closely to the time of exercise onset, a significant improvement in endurance was found. That participants only endured 21 minutes with the supplement or 13 minutes with the placebo indicates they were not well-trained athletes and raises questions as to the validity of the results.

A placebo-controlled study found that the daily use of a low-calorie energy drink paired with an exercise program resulted in improved exercise adaptations in a group of previously sedentary men (Lockwood et al., 2010). The exercise program included 3 days of cycling and 2 days of resistance training per week. Men in the study who received the energy drink made greater improvements in $\mathrm{VO}_{2 \max }$, body composition, and ventilatory threshold than men on the same training program who received a placebo. Furthermore, safety blood draws gathered over the ten weeks of the study showed that there was no compromised renal or hepatic function due to daily consumption of the energy drink. The energy drink in the study was called Celsius and contained a proprietary blend of caffeine, glucuronolactone, guaraná, taurine and other ingredients and contained only $10 \mathrm{kcal}$.

\section{Conclusion on Energy Drink Ingredients Effects on Aerobic Performance}

Caffeine in isolation reliably improves aerobic performance when consumed prior to exercise. Though carbohydrates before exercise have not always yielded ergogenic results, when 
ingested with caffeine, performance has been improved. Based on the literature ingestion of an energy drink containing at least $80 \mathrm{mg}$ of caffeine and $27 \mathrm{~g}$ of carbohydrate will improve aerobic performance if provided at least 30 minutes prior to exercise (Alford et al., 2001; Ivy et al., 2009). In these studies glucuronolactone, inositol, and taurine were all provided as well.

Glucuronolactone and taurine may improve the efficacy of caffeine and carbohydrate containing energy drinks (Gei $\beta$ et al., 1994) though the mechanisms of action are not understood. Ergogenic or ergolytic properties of ginseng, guaraná, and inositol have not been demonstrated.

\section{The Effects of Energy Drink Ingredients on Cognitive Performance}

\section{Caffeine Enhances VARious Aspects of Cognitive Function}

Early work that examined caffeine's effects on aerobic performance also reported that caffeine enhanced hand steadiness and tapping speeds in three subjects (Thornton et al., 1939). As noted before, this early work was flawed by modern standards for having a small sample size and was not statistically analyzed.

Caffeine is well-known to attenuate cognitive deficits that are the resultant of sleep deficits. Participants kept awake for over 40 hours performed better on tests of psychomotor vigilance and choice reaction time over the subsequent 12 hours if provided with $600 \mathrm{mg}$ of caffeine (Wesensten et al., 2002). Navy SEALs had improved visual vigilance and choice reaction time 1 and 8 hours after receiving 200-300 mg of caffeine when they had not slept in 72 hours (Lieberman et al., 2002). Pilots in the US Air Force had better performance on a tracking task, short-term memory, and visual vigilance if they received $200 \mathrm{mg}$ of caffeine every 4 hours during an overnight mission simulation (Doan et al., 2006).

Caffeine also boosts aspects of cognitive function in individuals who are not specifically sleep-deprived. A study of 110 volunteers revealed that individuals who reported the greatest daily caffeine intake had the best maintenance of simple reaction time from the beginning to the end of their workdays (Smith, 2005). Individuals who were low to moderate consumers of caffeine improved response time and executive control of attention during a flankers task when 
they ingested 100-400 mg of caffeine 30 minutes earlier (Brunyé et al., 2010). Individuals who regularly consumed high amounts of caffeine (greater than $550 \mathrm{mg}$ per day) only benefitted if they were given $400 \mathrm{mg}$ (Brunyé et al., 2010).

In doses as low as $60 \mathrm{mg}$, caffeine improved pattern recognition when taken 10 minutes before the task, and movement time when taken 40 minutes before the test (Durlach, 1998). In this work the caffeine was consumed as hot tea mixed with $20 \mathrm{ml}$ of part-skim milk, which contributed approximately $10 \mathrm{kcal}$ and less than $1 \mathrm{~g}$ carbohydrate. Follow-up research by the same group demonstrated that reaction time was reduced within minutes of consuming the lose dose (Durlach et al., 2002). While average caffeine intake of participants in the first study was not reported (Durlach, 1998), in the second study participants all consumed greater than $200 \mathrm{mg}$ of caffeine per day (Durlach et al., 2002). The rapid improvement in reaction time in this study may have been due to a reward response due to habitual consumption rather than a physiological effect of caffeine itself.

An even smaller dose of caffeine, $12.5 \mathrm{mg}$, resulted in improvements in simple reaction time from 2-66 minutes regardless of whether the participant was a low or high consumer of caffeine (Smit \& Rogers, 2000). Individuals who typically consumed large amounts of caffeine (207-532 $\mathrm{mg} /$ day) also had improvements in rapid visual information processing from 34-66 minutes. In addition to improving simple reaction time, $1.2 \mathrm{mg} / \mathrm{kg}$ of caffeine also enhanced mood, focus of attention, and lowered perception of difficulty of cognitive tasks following 8 hours of caffeine abstinence (Heatherley et al., 2005).

One study demonstrated that the cognitive benefits of caffeine extend into athletic reaction time (Duvnjak-Zaknich et al., 2011). The researchers demonstrated that caffeine in isolation enhanced the abilities of team-sports athletes to react to sports-specific stimuli before and after a simulated game. Specifically a $6 \mathrm{mg} / \mathrm{kg}$ dose of caffeine resulted in improved reactive agility times assessed by how fast athletes would change directions and sprint through the correct “exit" gate depending on a visual cue provided (see Figure 4). 


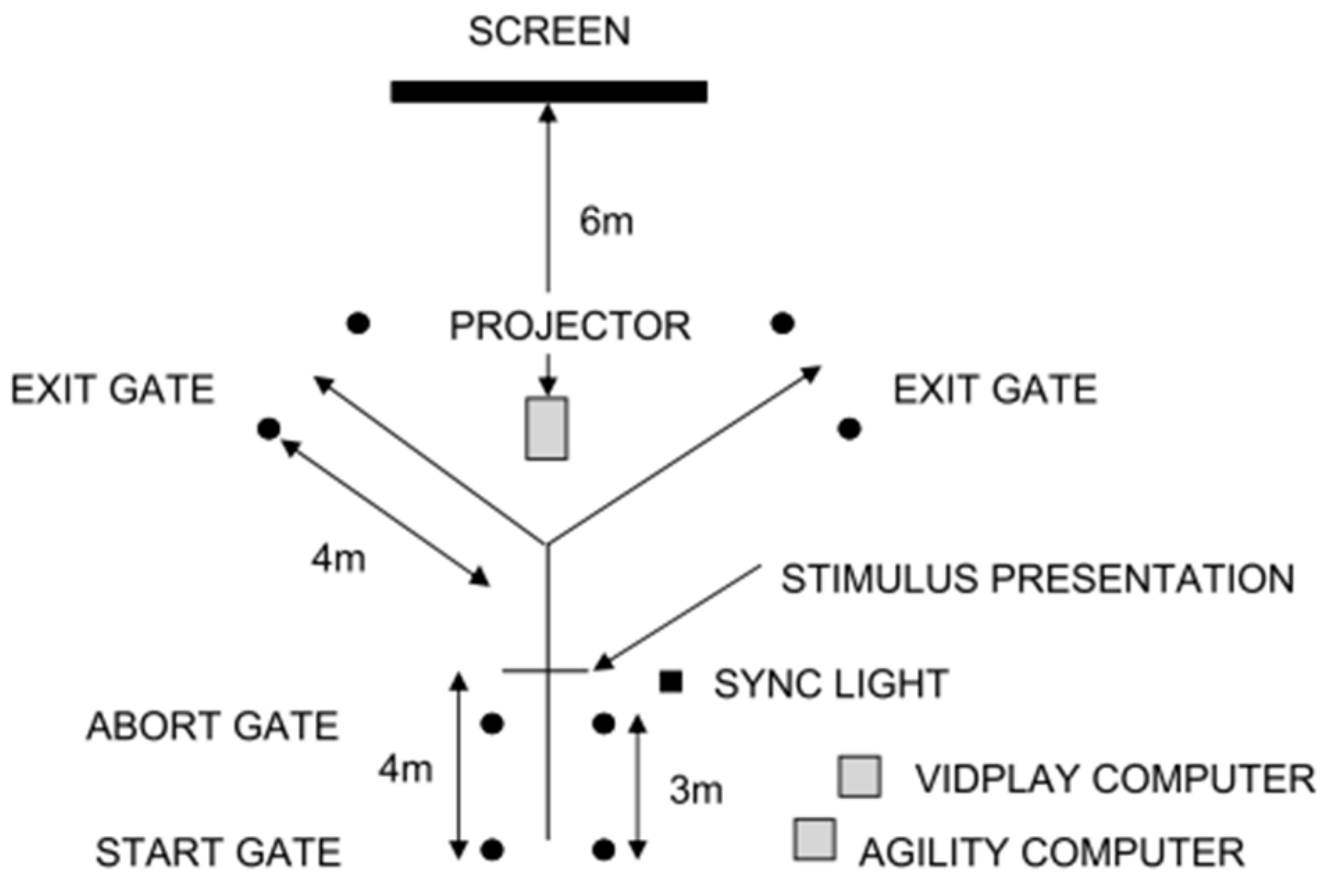

\section{VIDEO CAMERA}

Figure 4 From Duvnjak-Zaknich et al. (2011). Participants would sprint through the "start gate" until they passed the sync light. The sync light would initiate the projector to display an image of a player running to the left or right. The participant would then sprint through the corresponding "exit gate" and the completion time was recorded.

Caffeine has effects on response time that precipitate within ten minutes of ingestion, even when provided in doses as low as $60 \mathrm{mg}$ (Durlach, 1998; Durlach et al., 2002). If an individual has not had caffeine for at least 8 hours a dose of $1.2 \mathrm{mg} / \mathrm{kg}$ body mass improved mood, focus, and lowered perception of difficulty of cognitive tasks (Heatherley et al., 2005). When sleep-deprived, caffeine's effects become pronounced as it enhances visual vigilance and response time (Lieberman et al., 2002; Wesensten et al., 2002). Caffeine has also been shown to improve whole body choice reaction time when athletes are physically fatigued after a teamsports game simulation (Duvnjak-Zaknich et al., 2011).

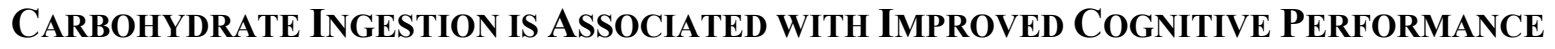

Breakfast is often touted as the most important meal of the day. In children, overnight and early morning fasting resulted in a variety of inhibited cognitive processes, including 
impaired memory and visual discrimination (Pollitt et al., 1998). In adults the skipping of breakfast also resulted in reduced memory though no effects were seen on intelligence quotient (Benton \& Parker, 1998; Benton \& Sargent, 1992). Regular feedings are likely necessary to maintain cognitive function as well. Reaction times were improved in physicians who were provided with balanced meals throughout their workdays compared to when they were not (Lemaire et al., 2010).

Conversely, other work did not show a difference in performance on several cognitive measures after 48 hours of restricted carbohydrate intake: there was no difference in performance on tests of visual vigilance, choice reaction time, or reasoning between individuals fed a normal diet, a diet consisting of predominantly carbohydrate, or a diet only contributing $\sim 20 \%$ of energy needs (H. R. Lieberman et al., 2008). The results from this study (H. R. Lieberman et al., 2008) seem to contradict those of other studies. Whereas other studies did not include a placebo group (Benton \& Parker, 1998; Benton \& Sargent, 1992), these researchers went so far as to create artificial foods that had comparable texture and taste to food that contributed more kcal. Further, this study is not directly comparable to those that use children as participants (Pollitt et al., 1998) since they are in a heightened state of metabolic demand due to growth.

Supplementations of carbohydrate enhance cognitive performance during tasks requiring high energy expenditure. US Army Rangers were able to maintain vigilance by responding to auditory stimuli with less delay over the course of a 10-hour physically demanding day if provided with supplemental carbohydrate rations (Lieberman et al., 2002). The soldiers who received $12 \%$ carbohydrate solution performed better on the vigilance task than those who received $6 \%$ solution, though both groups were in an energy deficit during the study. Also, after completing a protocol designed to simulate a team-sports competition, athletes performed better on a whole-body motor-skills test when they had ingested $128 \mathrm{~g}$ of carbohydrate over the course of exercise (Welsh et al., 2002).

Regardless of baseline blood glucose or absorptive state, when provided with $50 \mathrm{~g}$ or more of glucose memory has been found to be enhanced (Benton \& Owens, 1993). In direct 
contrast, an acute intake of 26 grams of carbohydrate in adults who were not fasted did not result in improved performance compared to energy-free placebo on measures of rapid visual information processing, reaction time, or memory in another study (Warburton et al., 2001). The apparent reason for the discrepancy in results is the carbohydrate provided: the lower amount of glucose provided in the second study may not have been sufficient to stimulate a cognitive enhancing effect.

While the findings of at least one study indicate that a lack of sufficient carbohydrate will not lead to cognitive detriments (H. R. Lieberman et al., 2008), most research indicates that regular meals improve cognition in normal daily tasks (Benton \& Parker, 1998; Lemaire et al., 2010; Pollitt et al., 1998). During physically demanding tasks carbohydrate intake specifically has been demonstrated to help attenuate cognitive decline (Lieberman et al., 2002; Welsh et al., 2002).

\section{OTHER ENERGY DRINK INGREDIENTS LACK SUFFICIENT RESEARCH TO CONCLUDE Cognition-Enhancing Properties}

\section{Ginseng}

A study using acute doses of ginseng found conflicting results on its efficacy to improve cognitive performance (Andrew et al., 2002). Specifically, participants performed no different or worse than placebo on a serial-subtraction task when they received 200 or $600 \mathrm{mg}$ of ginseng extract 1-2.5 or 6 hours prior to testing, though they performed better at 4-6 hours if they received a $400 \mathrm{mg}$ dose. The results were limited to when participants performed a serialsubtraction task using sevens, but there were no significant findings when the participants instead had to subtract by threes. More recent work by the same research group showed that acute administration of $200 \mathrm{mg}$ of ginseng delayed the onset of negative mood in participants but it also resulted in delayed responses to mental arithmetic (Reay et al., 2010). 
Ginseng paired with ginkgo biloba at "flavoring" concentrations did not result in differences in cognitive performance on simple reaction time, choice reaction time, word recall, or working memory (Scholey et al., 2004). Only $12.5 \mathrm{mg}$ of ginseng was provided in this study.

The research on panax ginseng in regards to cognitive function has been primarily focused on chronic supplementation on humans with cognitive deficits (Lee et al., 2008), or nonhumans (Petkov et al., 2003). The research demonstrating positive cognitive effects in healthy humans is inconsistent: see Lieberman (2001) for a review.

\section{Glucuronolactone}

Glucuronolactone is involved in glucuronidation of toxins in the liver and is normally produced in sufficient quantities via the PPP shunt (Eisenberg et al., 1959). One hour after taking $600 \mathrm{mg}$ glucuronolactone with $1000 \mathrm{mg}$ of taurine and $80 \mathrm{mg}$ of caffeine participants had better reaction time and attention during a stressful testing situation than when placebo was consumed (Seidl et al., 2000). Aside from this study, there is a lack of research on the effects of glucuronolactone on cognitive performance except when it is provided with other ingredients in an energy drink.

\section{Guaraná}

Participants receiving doses of $37.5 \mathrm{mg}$ or $75 \mathrm{mg}$ of guaraná extract (approximately $11 \%$ caffeine) 1 to 6 hours prior to cognitive testing had improvements in secondary memory compared to placebo, though no effects were seen for speed or accuracy of attention, speed of memory, or working memory (Haskell et al., 2007). Other research on the effectiveness of guaraná is limited in that guaraná is always co-administered with caffeine.

\section{Inositol}

Despite inositol's various physiological functions, there is no research on its effects on cognitive performance in humans. 


\section{Taurine}

As a stand-alone ingredient, chronic taurine supplementation as $1 \%$ of the total diet $(0.94$ $\mathrm{g} / \mathrm{kg}$ ) was found to be ineffective at improving learning and memory in Wistar rats (Ito et al., 2009). The rats in the study performed similarly to control rats in learning how to navigate a water maze. The research on taurine as a cognitive enhancing agent in humans is limited to investigations where it is combined with other ingredients in energy drinks.

\section{Conclusion on the Effects of Other Ingredients on Cognitive Performance}

Of the ingredients commonly added to energy drinks reviewed, only ginseng, guaraná, and taurine have research that evaluated effects on cognitive performance. The research is not convincing in that ginseng has produced mixed results (H. R. Lieberman, 2001), guaraná's effects are likely due to inevitable co-administration with caffeine, and taurine was unable to enhance learning when tested in rats (Ito et al., 2009).

\section{Energy Drinks Enhance Cognitive Performance Greater than Constituent INGREDIENTS}

When Red Bull energy drink was consumed 1 hour prior to testing, it enhanced attention and verbal reasoning compared to a carbohydrate-only control supplement or a placebo (Warburton et al., 2001). Notably, participants in this study were permitted to have caffeine ad libitum until the time of testing but still had performance enhancements due to the supplement. A double-dose of Red Bull (consisting of $160 \mathrm{mg}$ of caffeine, $54 \mathrm{~g}$ of carbohydrate, $2 \mathrm{mg}$ of taurine, and $1.2 \mathrm{~g}$ glucuronolactone) reduced lane drifting and enhanced reaction time during 2 hours of simulated driving compared to a control drink (Horne \& Reyner, 2001). A smaller dose of Red Bull given to participants prior to 4 hours of driving also reduced lane drifting and variations in vehicle speed (Mets et al., 2011). A dose-response study of Red Bull revealed that the greatest improvements on a go/no-go task were seen with a dose of $1.8 \mathrm{ml} / \mathrm{kg}$ body mass with improvements being reduced at higher dosages (Howard \& Marczinski, 2010). A dose of 1.8 $\mathrm{ml} / \mathrm{kg}$ would only result in $126 \mathrm{ml}$ for a $70 \mathrm{~kg}$ person, which is slightly more than half of the dose provided in a commercial volume of the beverage (Warburton et al., 2001) and 
approximately one quarter of the dose provided in other studies (Horne \& Reyner, 2001; Ivy et al., 2009).

An energy drink containing $75 \mathrm{mg}$ caffeine, $37.5 \mathrm{~g}$ glucose, and $12.5 \mathrm{mg}$ ginseng given to participants 30 minutes prior to cognitive testing resulted in enhancements in secondary memory and speed of attention (Scholey \& Kennedy, 2004). In this study participants receiving the caffeine or glucose in isolation did not perform better on any cognitive measure tested compared to placebo. This lead the investigators to conclude that the ingredients worked synergistically. When participants received $75 \mathrm{~g}$ glucose or $75 \mathrm{~g}$ caffeine they had enhanced simple reaction time, but when the ingredients were consumed together participants also had improved learning and consolidation of verbal memory (Adan \& Serra-Grabulosa, 2010).

It has been shown that a combination of caffeine and carbohydrate can enhance both physical and cognitive performance during glycogen-depleting exercise (Hogervorst et al., 2008). The participants ingested either $100 \mathrm{mg}$ of caffeine with $45 \mathrm{~g}$ of carbohydrate as a bar, the bar without caffeine, or a placebo drink immediately before starting a 2.5 hour ride at $60 \%$ of $\mathrm{VO}_{2 \max }$. During the ride participants received two more boluses of the treatment. At the end of the ride participants completed a ride to exhaustion at $75 \%$ of $\mathrm{VO}_{2 \max }$. Additionally participants completed cognitive testing twice during the 2.5 hour ride and once after the ride to exhaustion. The results of the study showed that when participants received the full supplement their aerobic performance (time to exhaustion) and reaction time during the cognitive tests were better than when they received the other two supplements The bar containing carbohydrate only resulted in improvements over the placebo beverage on both measures, as well.

\section{Conclusion on Energy Drink Ingredients Effects on Cognitive Performance}

Caffeine enhances cognitive performance in individuals who are sleep-deprived (Lieberman et al., 2002) and in doses as low as $60 \mathrm{mg}$ in rested individuals (Durlach et al., 2002). Caffeine and carbohydrate appear to have a synergistic effect on cognitive performance (Adan \& Serra-Grabulosa, 2010; Kennedy \& Scholey, 2004), even during and after a prolonged 
moderate-intensity exercise (Hogervorst et al., 2008). The addition of glucuronolactone and taurine may further enhance the cognitive effects of caffeine and carbohydrate mixtures, as Red Bull energy drink has been shown to enhance cognitive performance (Horne \& Reyner, 2001; Howard \& Marczinski, 2010; Mets et al., 2011; Warburton et al., 2001).

\section{Aerobic Exercise Enhances Reaction Time without a Detriment to other Cognitive Processes}

Prolonged endurance exercise does not appear to impair cognitive performance. Prolonged low-intensity walking activity had no effect on performance of simple psychomotor tasks (Ivy et al., 1983). Runners performed to the same level on a Stroop color-word test before and after completing either a 30-km race or a marathon (Blomstrand et al., 1991).

Enhancements in cognitive performance due to exercise have been observed when tested under sport-specific settings. For example, McMorris and Graydon (1997) presented soccer players with visual images of game or non-game situations where a soccer ball was or was not present. The images were presented for only two seconds and the participants' task was to respond with a "yes" if a ball was present or a "no" if a ball was not present. The primary findings from the study were that when players were exercising at maximum intensity on a cycle ergometer they had improved speed of decision without a loss of accuracy, though the results were equivocal when the exercise was at $70 \% \mathrm{VO}_{2 \max }$. When the researchers made the task more complex by instructing participants to make a decision to pass, shoot, dribble, or run, maximal intensity exercise also increased accuracy of decision making compared to when participants were at rest. The authors concluded that the maximum exercise intensity invoked a greater release of catecholamines that were responsible for the improvement in decision-making time.

Follow-up work by the same researchers tested soccer players using the same testing protocol but examined performance when participants were at rest, exercising at the "adrenaline threshold", or exercising at $\mathrm{VO}_{2 \max }$ (McMorris et al., 1999). The "adrenaline threshold" was determined as the point at which blood epinephrine increased above baseline values during an 
incremental exercise test (see Figure 5). Similar to their previous work, researchers found that decision making time was enhanced when participants were exercising at their maximal aerobic capacity. The researchers also found that speed of decision making was enhanced at the "adrenaline threshold" although this corresponded to a lower workload than their previous work $\left(56 \%\right.$ of $\mathrm{VO}_{2 \max }$ compared to $\left.70 \%\right)$.
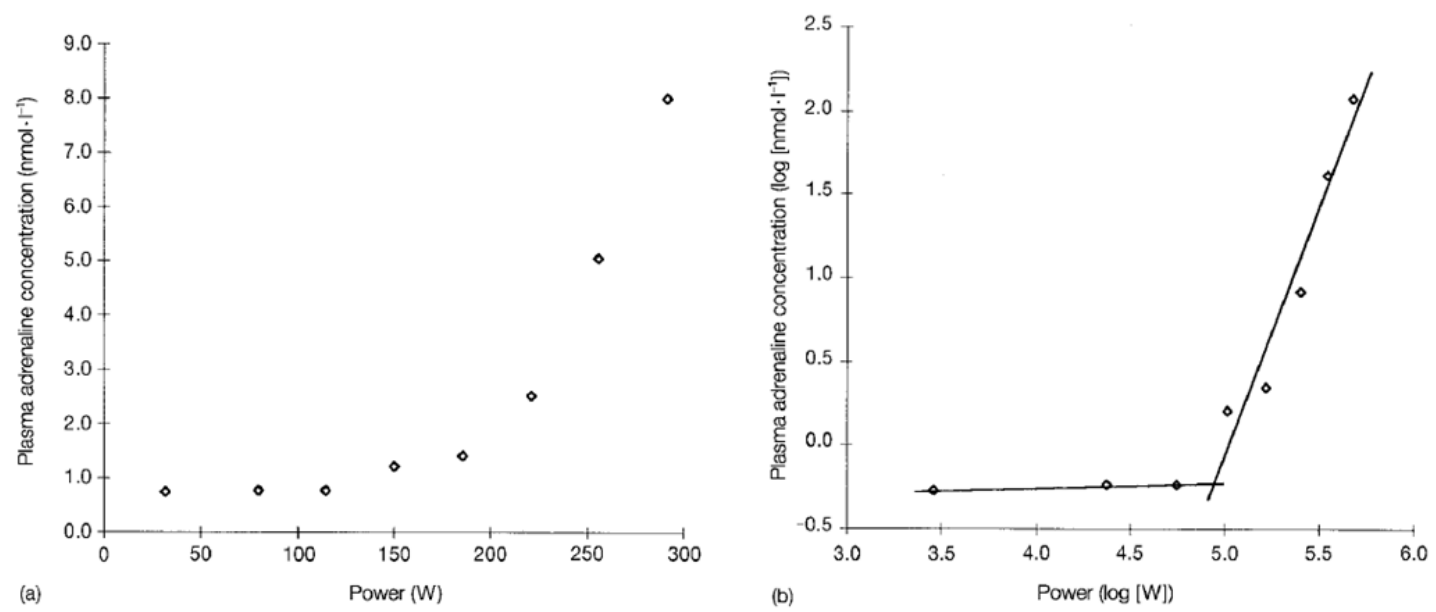

Figure 5 From T. McMorris et al. (1999). "Adrenaline threshold" was defined as the point at which blood epinephrine concentrations increase disproportionately to increased workload during an incremental exercise test.

In order to make the cognitive tasks more ecologically valid, the researchers modified the protocol so that correct reactions included passing a ball to a target in front of the athlete instead of simply verbalizing a cognitive decision as in previous iterations of the research (McMorris et al., 2000). No differences in reaction time or accuracy of decision-making were seen due to the exercise at any intensity compared to rest. The explanation for the loss in an exercise effect on cognitive performance may be due to study design: in previous versions of the research the players verbalized cognitive decisions while cycling on an ergometer (McMorris et al., 1999; McMorris \& Graydon, 1997), while in this study the participants rode on a cycle ergometer until reaching the necessary exercise intensity but dismounted the ergometer and were no longer exercising when the cognitive decision was made.

To make the exercise condition more similar to what is actually experienced by soccer players, the investigators tested the effects of repeated sprints on passing frequency and accuracy 
(McMorris \& Rayment, 2007). The researchers saw a linear decrease in passing accuracy, an inverted-U shaped response in passing frequency, and a linear increase in both HR and RPE as athletes went from resting to three consecutive 100-meter sprints (see Figure 6).

1

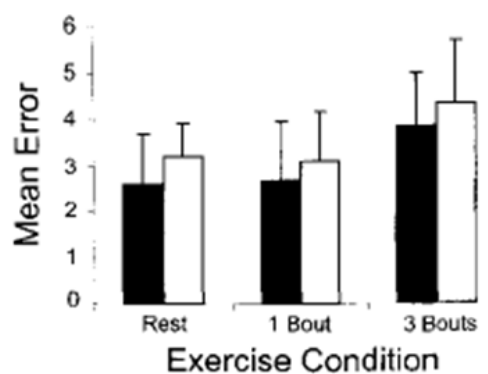

3

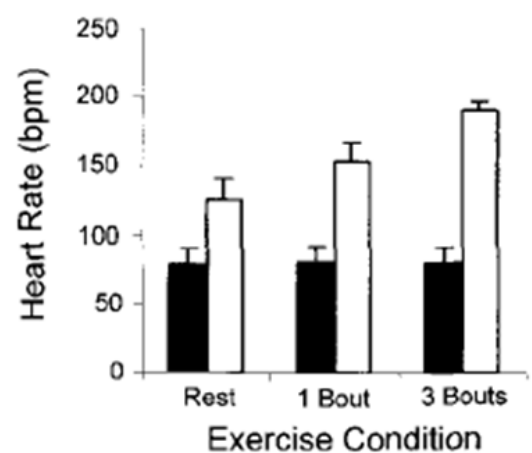

2

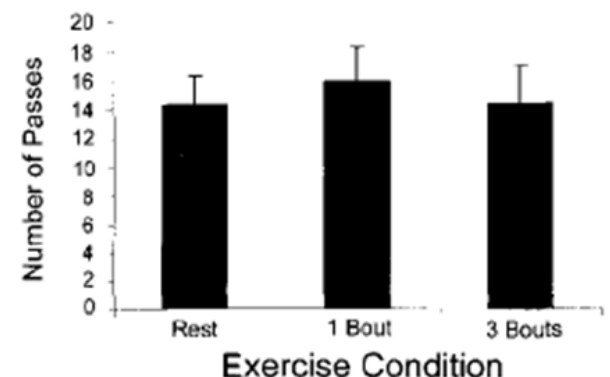

4

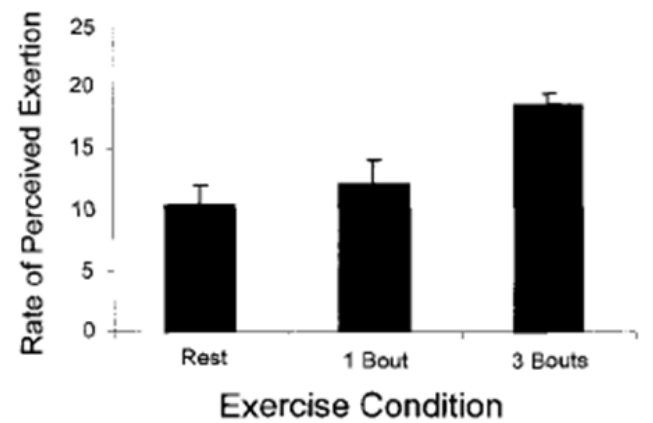

Figure 6 From Terry McMorris \& Rayment (2007). The researchers found an increase in gross motor skill performance as passing errors increased from rest to three sprints of $100-\mathrm{m}$. The number of passes increased after one sprint, but returned to resting levels after three. HR and RPE both increased with higher levels of exercise.

Another study was conducted to assess the effects that aerobic exercise had on tests designed to measure executive function and memory (Coles \& Tomporowski, 2008). Researchers found no benefit of completing 40 minutes of low-moderate intensity exercise (10 minutes of $30 \% \mathrm{VO}_{2 \max }$ and 30 minutes of $60 \% \mathrm{VO}_{2 \max }$ ) on measures of executive function as determined by a set-switching task and a Brown-Peterson test, or in overall memory as determined by word recall tasks. The researchers did report that there was an improvement due to exercise in recalling words in the primacy and recency portions of the word lists that participants were presented with.

It has been demonstrated that during exercise individuals become more acutely sensitive to visual cues while performance on executive function tasks remain unchanged (Lambourne et al., 2010). Participants exercised on a cycle ergometer for 40 minutes at $90 \%$ of ventilatory 
threshold (VT) and at 5 times during the workout completed a critical flicker fusion (CFF) test and paced auditory serial addition task (PASAT). Performance on the CFF increased as the exercise time increased and was significantly different than a resting control by 35 and 40 minutes of exercise.

Another study demonstrated an improvement in responses to visual stimuli during exercise (Sanabria et al., 2011). Participants performed better on a test of exogenous spatial attention when exercising for 20 minutes at $85 \%$ of their anaerobic threshold compared to performing the same test while at rest. The participants also performed better on the test immediately after exercise compared to resting performance.

The effects of acute bouts of exercise on cognitive processes are mixed. Exercise enhances speed of reaction (McMorris et al., 1999; McMorris \& Graydon, 1997; McMorris et al., 2011) as well as simple sensitivity to visual stimuli (Lambourne, Audiffren, \& Tomporowski, 2010b; Sanabria et al., 2011), though complex motor skill performance may be hindered (McMorris et al., 2011) and executive function does not appear to be affected (Coles \& Tomporowski, 2008; Lambourne, Audiffren, \& Tomporowski, 2010b). Prolonged aerobic exercise leading to dehydration will lead to cognitive deficits though shorter bouts may have positive effects on cognitive processes (Tomporowski, 2003).

\section{Conclusion}

The combination of caffeine and carbohydrate has been demonstrated to enhance both aerobic and cognitive performance when ingested immediately prior to and throughout glycogen depleting exercise (Hogervorst et al., 2008). It has not been shown if the addition of other energy drink components will result in further enhancements during this type of exercise. Nor has it been shown if similar effects can be observed during or after an exercise that is of shorter duration and higher intensity (e.g., a one-hour time-trial cycling race). Finally, given that many athletic events are structured in a manner that make continued supplementation during competition difficult to achieve, it is worthwhile to evaluate the effectiveness of enhancing 
aerobic and cognitive performance when an energy drink is delivered in the hour prior to exercise.

Red Bull energy drink (containing caffeine, carbohydrate, glucuronolactone, and taurine) has been proven effective in enhancing aerobic performance (Alford et al., 2001; Ivy et al., 2009) as well as cognitive performance (Horne \& Reyner, 2001; Howard \& Marczinski, 2010; Mets et al., 2011; Warburton et al., 2001). Ginseng's effects on cognition are unclear, but are likely to be nonnegative (H. R. Lieberman, 2001) while it has not been studied as an ergogenic aid. Guaraná has been shown to enhance secondary memory in one study (Haskell et al., 2007), though comprehensive research on its effects on cognition or exercise is lacking. Similarly, there is no research on inositol's unique effects on exercise or cognitive performance.

When taken prior to a 1-hour bout of moderate- to high-intensity exercise, an energy drink containing caffeine, carbohydrate, ginseng, glucuronolactone, guaraná, inositol, and taurine will likely serve as an ergogenic aid while also enhancing cognitive performance. 


\section{APPENDIX A: DESCRIPTIVE AND INFERENTIAL STATISTICS}

Table 1: Participant Characteristics

\begin{tabular}{|c|c|c|c|c|c|c|}
\hline & \multicolumn{2}{|c|}{ Females (N=7) } & \multicolumn{2}{c|}{ Males (N=8) } & \multicolumn{2}{c|}{ Total (N=15) } \\
& $\overline{\mathbf{X}}$ & $\mathbf{S E}_{\overline{\mathbf{X}}}$ & $\overline{\mathbf{X}}$ & $\mathbf{S E}_{\overline{\mathbf{X}}}$ & $\overline{\mathbf{X}}$ & $\mathbf{S E}_{\overline{\mathbf{X}}}$ \\
\hline Age (yr.) & 30 & 2.3 & 31 & 2.5 & 31 & 1.6 \\
\hline Mass (kg) & 65.7 & 2.50 & 69.7 & 3.68 & 67.8 & 2.27 \\
\hline Height (cm) & 171 & 1.8 & 177 & 2.7 & 174 & 1.7 \\
\hline VO $_{\text {2MAX (mL/min) }}$ & 3407 & 88.3 & 4251 & 201.1 & 3857 & 158.1 \\
\hline IQ & 122 & 3.8 & 112 & 4.5 & 117 & 3.2 \\
\hline
\end{tabular}

Table 2: Time to Finish (seconds)

\begin{tabular}{|c|c|c|}
\hline$(\mathbf{N}=15)$ & $\overline{\mathbf{X}}$ & $\mathbf{S E}_{\overline{\mathbf{X}}}$ \\
\hline ED & $3848.5^{*}$ & 78.12 \\
\hline PLA & 3968.9 & 80.56 \\
\hline \multicolumn{3}{|c|}{ *Effect found for treatment $(\mathrm{p}<.05)}$. \\
\hline
\end{tabular}

Table 3: Gas Collection

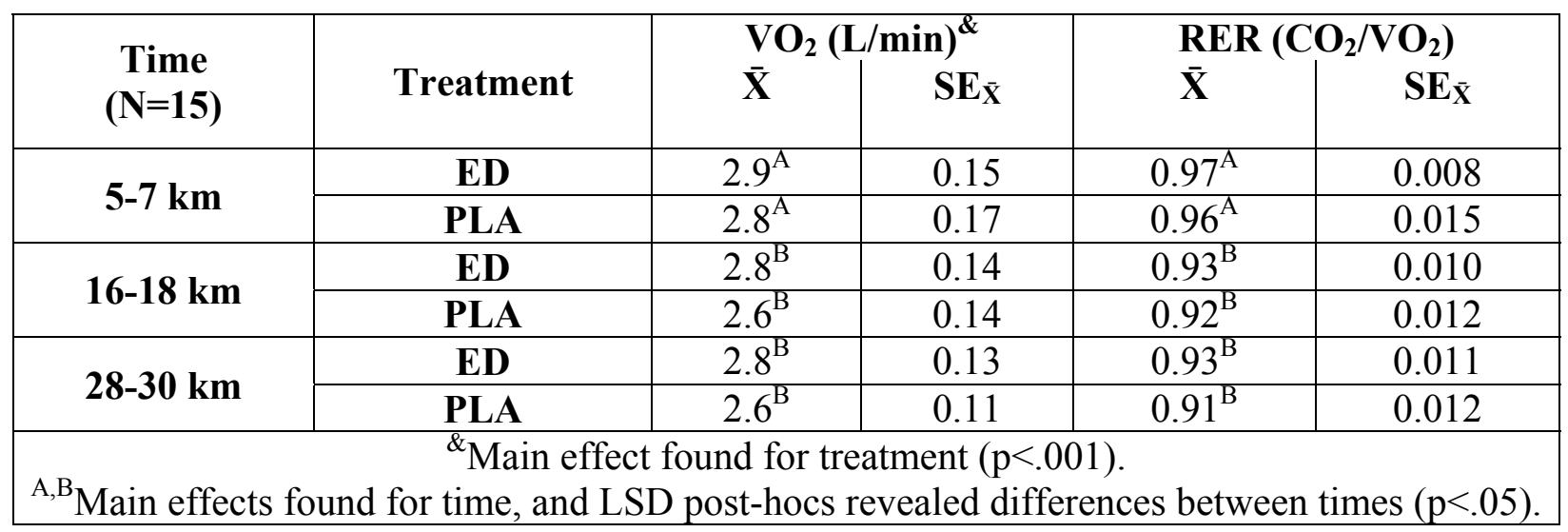


Table 4: Heart Rate

\begin{tabular}{|c|c|c|c|}
\hline $\begin{array}{c}\text { Time } \\
(\mathbf{N}=9)^{\wedge}\end{array}$ & Treatment $^{\&}$ & $\overline{\mathbf{X}}$ & $\mathbf{S E}_{\overline{\mathbf{X}}}$ \\
\hline \multirow{2}{*}{ Pre Drink Pre Race } & ED & 58 & 2.3 \\
\hline & PLA & 60 & 2.7 \\
\hline \multirow{2}{*}{ Post Drink Pre Race } & ED & 58 & 2.6 \\
\hline & PLA & 56 & 2.5 \\
\hline \multirow{2}{*}{$1 \mathrm{~km}$} & ED & 153 & 4.4 \\
\hline & PLA & 150 & 4.9 \\
\hline \multirow{2}{*}{$6 \mathrm{~km}$} & ED & 165 & 3.2 \\
\hline & PLA & 162 & 4.3 \\
\hline \multirow{2}{*}{$12 \mathrm{~km}$} & ED & 172 & 3.4 \\
\hline & PLA & 167 & 4.6 \\
\hline \multirow{2}{*}{17 km } & ED & 166 & 3.2 \\
\hline & PLA & 163 & 3.9 \\
\hline \multirow{2}{*}{$23 \mathrm{~km}$} & ED & 169 & 3.0 \\
\hline & PLA & 162 & 3.9 \\
\hline \multirow{2}{*}{29 km } & ED & 169 & 2.4 \\
\hline & PLA & 160 & 2.6 \\
\hline \multirow{2}{*}{34 km } & ED & 171 & 3.2 \\
\hline & PLA & 165 & 2.4 \\
\hline \multirow{2}{*}{ Start of Stroop } & ED & 167 & 3.6 \\
\hline & PLA & 163 & 3.8 \\
\hline \multirow{2}{*}{30 seconds of Stroop } & ED & 163 & 3.1 \\
\hline & PLA & 157 & 3.3 \\
\hline \multirow{2}{*}{60 seconds of Stroop } & ED & 161 & 3.2 \\
\hline & PLA & 160 & 2.2 \\
\hline \multirow{2}{*}{90 seconds of Stroop } & ED & 163 & 2.7 \\
\hline & PLA & 161 & 2.5 \\
\hline \multirow{2}{*}{120 seconds of Stroop } & ED & 163 & 2.8 \\
\hline & PLA & 161 & 2.6 \\
\hline
\end{tabular}


Table 5: RPE (Borg 6-20)

\begin{tabular}{|c|c|c|c|}
\hline $\begin{array}{c}\text { Time } \\
(\mathrm{N}=15)\end{array}$ & Treatment & $\overline{\mathbf{X}}$ & $\mathbf{S E}_{\overline{\mathbf{X}}}$ \\
\hline \multirow{2}{*}{$1 \mathbf{k m}^{\mathrm{A}}$} & ED & 12 & 0.5 \\
\hline & PLA & 12 & 0.5 \\
\hline \multirow{2}{*}{$6 \mathrm{~km}^{\mathrm{B}}$} & ED & 14 & 0.3 \\
\hline & PLA & 14 & 0.4 \\
\hline \multirow{2}{*}{$12 \mathrm{~km}^{\mathrm{CF}}$} & ED & 16 & 0.3 \\
\hline & PLA & 15 & 0.4 \\
\hline \multirow{2}{*}{$17 \mathbf{k m}^{\mathrm{D}}$} & ED & 15 & 0.4 \\
\hline & PLA & 15 & 0.4 \\
\hline \multirow{2}{*}{$23 \mathrm{~km}^{\mathrm{EF}}$} & ED & 16 & 0.4 \\
\hline & PLA & 16 & 0.5 \\
\hline \multirow{2}{*}{$29 \mathrm{~km}^{\mathrm{CEF}}$} & ED & 16 & 0.4 \\
\hline & PLA & 16 & 0.5 \\
\hline \multirow{2}{*}{$34 \mathrm{~km}^{\mathrm{G}}$} & ED & 17 & 0.4 \\
\hline & PLA & 17 & 0.4 \\
\hline
\end{tabular}


Table 6: Executive Function Test

\begin{tabular}{|c|c|c|c|c|c|c|c|c|c|}
\hline \multirow{2}{*}{$\begin{array}{c}\text { Time } \\
(\mathrm{N}=15)\end{array}$} & \multirow{2}{*}{ Treatment } & \multicolumn{2}{|c|}{$\begin{array}{l}\text { Taps per } \\
\text { Second }^{\&}\end{array}$} & \multicolumn{2}{|c|}{$\begin{array}{c}\text { Reaction } \\
\text { Time } \\
\text { (milliseconds) }\end{array}$} & \multicolumn{2}{|c|}{$\begin{array}{l}\text { Movement Time } \\
\text { (milliseconds) }\end{array}$} & \multicolumn{2}{|c|}{$\begin{array}{c}\text { Total Time } \\
(\text { milliseconds) }\end{array}$} \\
\hline & & $\overline{\mathbf{X}}$ & $\mathbf{S E}_{\overline{\mathbf{X}}}$ & $\overline{\mathbf{X}}$ & $\overline{\mathbf{X}}$ & $\overline{\mathbf{X}}$ & $\mathbf{S E}_{\overline{\mathbf{X}}}$ & $\overline{\mathbf{X}}$ & $\mathbf{S E}_{\overline{\mathbf{X}}}$ \\
\hline \multirow{2}{*}{$\begin{array}{c}\text { Pre } \\
\text { Drink } \\
\text { Pre } \\
\text { Race }\end{array}$} & ED & $5.8^{\mathrm{A}}$ & 0.18 & 535 & 18.7 & $347^{\mathrm{A}}$ & 24.4 & 881 & 41.0 \\
\hline & PLA & $5.6^{\mathrm{A}}$ & 0.20 & 556 & 26.9 & $348^{\mathrm{A}}$ & 29.4 & 904 & 53.0 \\
\hline \multirow{2}{*}{$\begin{array}{c}\text { Post } \\
\text { Drink } \\
\text { Pre } \\
\text { Race } \\
\end{array}$} & ED & $5.9^{\mathrm{A}}$ & 0.21 & 522 & 20.0 & $336^{\mathrm{AB}}$ & 24.2 & 858 & 33.0 \\
\hline & PLA & $5.4^{\mathrm{A}}$ & 0.18 & 558 & 23.0 & $345^{\mathrm{AB}}$ & 25.6 & 903 & 42.2 \\
\hline \multirow{2}{*}{$\begin{array}{l}\text { Post } \\
\text { Race }\end{array}$} & ED & $6.2^{\mathrm{B}}$ & 0.18 & 507 & 21.3 & $319^{\mathrm{BC}}$ & 22.7 & 826 & 35.9 \\
\hline & PLA & $5.9^{\mathrm{B}}$ & 0.18 & 542 & 32.0 & $336^{\mathrm{BC}}$ & 28.2 & 878 & 52.5 \\
\hline$A-C_{D}$ & & ${ }^{\&} \mathrm{~N}$ & $\operatorname{ain} \mathrm{e}$ & ct $w$ & ound & $\begin{array}{l}\text { treat } \\
\text {-hocs }\end{array}$ & $p<.05)$. & het & mes \\
\hline
\end{tabular}

Table 7: Tap Test

\begin{tabular}{|c|c|c|c|c|c|}
\hline \multirow[b]{2}{*}{ Time } & \multirow[b]{2}{*}{ Treatment } & \multicolumn{2}{|c|}{$\begin{array}{l}\text { Percent of Precise Responses }{ }^{\&} \\
(\mathrm{~N}=11)^{\wedge}\end{array}$} & \multicolumn{2}{|c|}{ Taps Per Second } \\
\hline & & $\overline{\mathbf{X}}$ & $\mathbf{S E}_{\overline{\mathbf{X}}}$ & $\overline{\mathbf{X}}$ & $\mathbf{S E}_{\overline{\mathbf{X}}}$ \\
\hline \multirow{2}{*}{ Pre Drink Pre Race } & ED & 99.8 & 0.08 & 3.7 & 0.13 \\
\hline & PLA & 99.8 & 0.15 & 3.7 & 0.11 \\
\hline \multirow{2}{*}{ Post Drink Pre Race } & ED & 99.5 & 0.22 & $3.7 *$ & 0.12 \\
\hline & PLA & 99.6 & 0.10 & 3.4 & 0.09 \\
\hline \multirow{2}{*}{ Post Race } & ED & 99.2 & 0.41 & $3.9^{*}$ & 0.09 \\
\hline & PLA & 99.6 & 0.25 & 3.8 & 0.11 \\
\hline \multicolumn{6}{|c|}{ 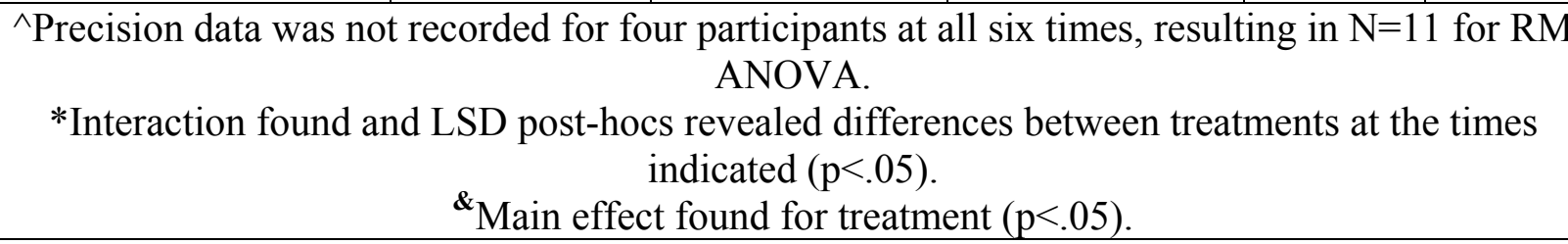 } \\
\hline
\end{tabular}


Table 8: Stroop Test

\begin{tabular}{|c|c|c|c|c|c|c|c|c|c|c|c|c|c|}
\hline \multirow{3}{*}{$\begin{array}{c}\text { Time } \\
(\mathrm{N}=15)\end{array}$} & \multirow{3}{*}{ Treatment } & \multicolumn{4}{|c|}{ "Read" stimuli } & \multicolumn{4}{|c|}{ "Incongruent" stimuli } & \multicolumn{4}{|c|}{ Total (both stimuli types) } \\
\hline & & \multicolumn{2}{|c|}{$\begin{array}{l}\text { Percent of } \\
\text { Correct } \\
\text { Responses }\end{array}$} & \multicolumn{2}{|c|}{$\begin{array}{l}\text { Reaction Time } \\
\text { (seconds) }\end{array}$} & \multicolumn{2}{|c|}{$\begin{array}{c}\text { Percent of } \\
\text { Correct } \\
\text { Responses }\end{array}$} & \multicolumn{2}{|c|}{$\begin{array}{l}\text { Reaction Time } \\
\text { (seconds) }\end{array}$} & \multicolumn{2}{|c|}{$\begin{array}{c}\text { Percent of } \\
\text { Correct } \\
\text { Responses }\end{array}$} & \multicolumn{2}{|c|}{$\begin{array}{l}\text { Reaction Time } \\
\text { (seconds) }\end{array}$} \\
\hline & & $\overline{\mathbf{X}}$ & $\mathbf{S E}_{\overline{\mathbf{X}}}$ & $\overline{\mathbf{X}}$ & $\mathbf{S E}_{\overline{\mathbf{X}}}$ & $\overline{\mathbf{X}}$ & $\mathbf{S E}_{\overline{\mathbf{X}}}$ & $\overline{\mathbf{X}}$ & $\mathbf{S E}_{\overline{\mathbf{X}}}$ & $\overline{\mathbf{X}}$ & $\mathbf{S E}_{\overline{\mathbf{X}}}$ & $\overline{\mathbf{X}}$ & $\mathbf{S E}_{\overline{\mathbf{X}}}$ \\
\hline \multirow{2}{*}{$\begin{array}{c}\text { Pre Drink } \\
\text { Pre Race }\end{array}$} & ED & 92.4 & 3.30 & 1.18 & 0.088 & 98.5 & 0.69 & $1.10^{\mathrm{A}}$ & 0.071 & 95.9 & 1.26 & $1.14^{\mathrm{A}}$ & 0.070 \\
\hline & PLA & 90.8 & 6.64 & 1.12 & 0.117 & 98.0 & 0.91 & $1.11^{\mathrm{A}}$ & 0.062 & 97.6 & 0.99 & $1.14^{\mathrm{A}}$ & 0.070 \\
\hline \multirow{2}{*}{ Post Race } & ED & 92.6 & 2.84 & 1.09 & 0.064 & 96.8 & 1.30 & $0.99^{\mathrm{B}}$ & 0.061 & 95.3 & 1.26 & $1.03^{\mathrm{B}}$ & 0.058 \\
\hline & PLA & 92.3 & 4.84 & 1.07 & 0.069 & 96.1 & 1.46 & $1.03^{\mathrm{B}}$ & 0.051 & 94.9 & 1.81 & $1.04^{\mathrm{B}}$ & 0.058 \\
\hline
\end{tabular}


Table 9: Reaction Time Test

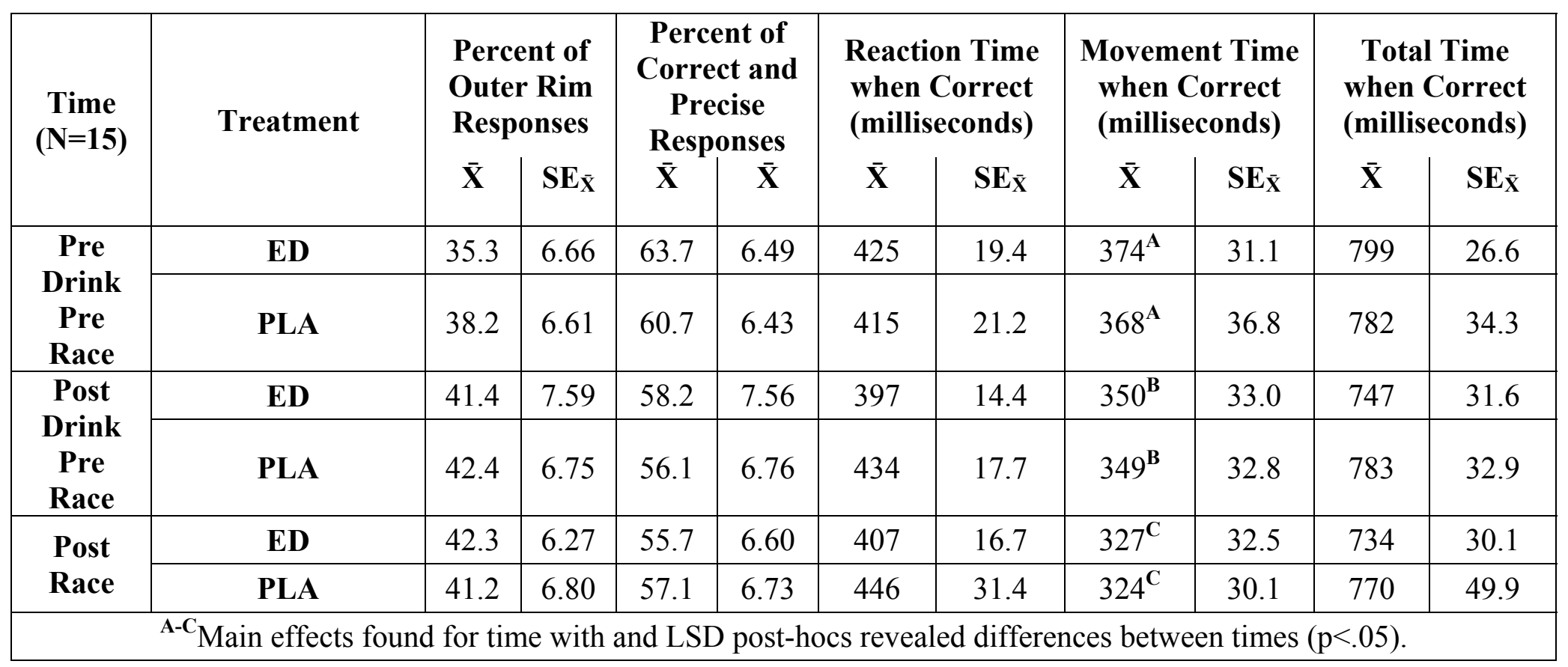


Table 5: Blood Measures

\begin{tabular}{|c|c|c|c|c|c|c|c|c|c|c|c|c|c|}
\hline \multirow{2}{*}{$\begin{array}{c}\text { Time } \\
(\mathbf{N}=15)\end{array}$} & \multirow{2}{*}{ Treatment } & \multicolumn{2}{|c|}{$\begin{array}{l}\text { Insulin } \\
(\mu \mathrm{U} / \mathrm{mL})\end{array}$} & \multicolumn{2}{|c|}{$\begin{array}{l}\text { Glucose } \\
\text { (mg/dL) }\end{array}$} & \multicolumn{2}{|c|}{$\begin{array}{c}\text { Free Fatty Acids } \\
(\mathrm{mmol} / \mathrm{L})\end{array}$} & \multicolumn{2}{|c|}{$\begin{array}{l}\text { Glycerol } \\
(\mathrm{mmol} / \mathrm{L})\end{array}$} & \multicolumn{2}{|c|}{$\begin{array}{l}\text { Lactate } \\
(\mathrm{mmol} / \mathrm{L})\end{array}$} & \multicolumn{2}{|c|}{$\begin{array}{l}\text { Myoglobin } \\
\text { (ng/mL) }\end{array}$} \\
\hline & & $\overline{\mathbf{X}}$ & $\mathbf{S E}_{\overline{\mathbf{X}}}$ & $\overline{\mathbf{X}}$ & $\mathbf{S E}_{\overline{\mathbf{X}}}$ & $\overline{\mathbf{X}}$ & $\mathbf{S E}_{\overline{\mathbf{X}}}$ & $\overline{\mathbf{X}}$ & $\mathbf{S E}_{\overline{\mathbf{X}}}$ & $\overline{\mathbf{X}}$ & $\mathbf{S E}_{\overline{\mathbf{X}}}$ & $\overline{\mathbf{X}}$ & $\mathbf{S E}_{\overline{\mathbf{X}}}$ \\
\hline \multirow{2}{*}{$\begin{array}{c}\text { Pre Drink } \\
\text { Pre Race }\end{array}$} & ED & 27 & 3.3 & 83 & 1.5 & 0.29 & 0.032 & $0.05^{\mathrm{A}}$ & 0.006 & 0.7 & 0.05 & $18^{\mathrm{ABCDE}}$ & 2.6 \\
\hline & PLA & 26 & 2.0 & 83 & 1.4 & 0.25 & 0.020 & $0.05^{\mathrm{A}}$ & 0.006 & 0.7 & 0.03 & $19^{\mathrm{ABCDE}}$ & 3.6 \\
\hline \multirow{2}{*}{$\begin{array}{c}\text { Post Drink } \\
\text { Pre Race }\end{array}$} & ED & $63 *$ & 5.9 & 82 & 5.5 & $0.13 *$ & 0.014 & $0.04^{\mathrm{A}}$ & 0.003 & $1.7 *$ & 0.08 & $19^{\mathrm{ABD}}$ & 3.3 \\
\hline & PLA & 27 & 3.0 & 77 & 1.5 & 0.21 & 0.020 & $0.06^{\mathrm{A}}$ & 0.008 & 0.7 & 0.03 & $18^{\mathrm{ABD}}$ & 4.0 \\
\hline \multirow{2}{*}{$6 \mathrm{~km}$} & ED & 26 & 2.5 & $63 *$ & 3.5 & $0.10^{*}$ & 0.013 & $0.13^{\mathrm{B}}$ & 0.016 & $4.8^{*}$ & 0.38 & $17^{\mathrm{AC}}$ & 3.3 \\
\hline & PLA & 22 & 2.4 & 79 & 2.0 & 0.19 & 0.019 & $0.16^{\mathrm{B}}$ & 0.023 & 3.5 & 0.49 & $17^{\mathrm{AC}}$ & 3.8 \\
\hline \multirow{2}{*}{17 km } & ED & 20 & 1.3 & $76^{*}$ & 2.6 & $0.14^{*}$ & 0.021 & $0.26^{\mathrm{C}}$ & 0.030 & $4.8^{*}$ & 0.48 & & \\
\hline & PLA & 23 & 2.5 & 85 & 1.7 & 0.26 & 0.029 & $0.27^{\mathrm{C}}$ & 0.038 & 3.1 & 0.50 & & \\
\hline \multirow{2}{*}{$23 \mathrm{~km}$} & ED & 20 & 1.6 & $76^{*}$ & 2.3 & $0.17^{*}$ & 0.028 & $0.31^{\mathrm{D}}$ & 0.037 & $4.3 *$ & 0.47 & $19^{\mathrm{ABDE}}$ & 4.4 \\
\hline & PLA & 21 & 1.5 & 82 & 1.9 & 0.27 & 0.027 & $0.30^{\mathrm{D}}$ & 0.040 & 2.6 & 0.38 & $20^{\mathrm{ABDE}}$ & 4.5 \\
\hline \multirow{2}{*}{29 km } & ED & 18 & 1.2 & 74 & 2.1 & 0.22 & 0.042 & $0.35^{\mathrm{E}}$ & 0.042 & $4.1^{*}$ & 0.34 & $20^{\mathrm{ABDE}}$ & 3.3 \\
\hline & PLA & 18 & 1.9 & 75 & 2.2 & 0.28 & 0.036 & $0.33^{\mathrm{E}}$ & 0.045 & 2.4 & 0.34 & $21^{\mathrm{ABDE}}$ & 4.9 \\
\hline \multirow{2}{*}{34 km } & ED & 19 & 2.5 & 70 & 2.6 & 0.24 & 0.049 & $0.38^{\mathrm{F}}$ & 0.045 & $4.1^{*}$ & 0.38 & $22^{\mathrm{ADE}}$ & 4.3 \\
\hline & PLA & 19 & 2.5 & 74 & 2.1 & 0.30 & 0.039 & $0.36^{\mathrm{F}}$ & 0.046 & 2.6 & 0.44 & $21^{\mathrm{ADE}}$ & 4.0 \\
\hline
\end{tabular}


Table 6: Blood Measures (continued)

\begin{tabular}{|c|c|c|c|c|c|c|c|c|c|c|c|}
\hline \multirow[t]{2}{*}{ Time } & \multirow[t]{2}{*}{ Treatment } & \multicolumn{2}{|c|}{$\begin{array}{c}\text { Caffeine } \\
(\mathbf{n g} / \mathbf{m L}) \\
(\mathbf{N}=15)\end{array}$} & \multicolumn{2}{|c|}{$\begin{array}{c}\text { Epinephrine } \\
(\mathrm{pg} / \mathrm{mL} \mathbf{)} \\
(\mathrm{N}=15)\end{array}$} & \multicolumn{2}{|c|}{$\begin{array}{c}\text { Norepinephrine } \\
(\mathrm{pg} / \mathbf{m L}) \\
(\mathrm{N}=15)\end{array}$} & \multicolumn{2}{|c|}{$\begin{array}{c}\beta \text {-Endorphins } \\
(\mathrm{ng} / \mathbf{m L}) \\
(\mathbf{N}=14)^{\wedge}\end{array}$} & \multicolumn{2}{|c|}{$\begin{array}{l}\text { Cortisol } \\
(\mu \mathrm{g} / \mathrm{dL}) \\
(\mathrm{N}=15)\end{array}$} \\
\hline & & $\overline{\mathbf{X}}$ & $\mathbf{S E}_{\overline{\mathbf{X}}}$ & $\overline{\mathbf{X}}$ & $\mathbf{S E}_{\overline{\mathbf{X}}}$ & $\overline{\mathbf{X}}$ & $\mathbf{S E}_{\overline{\mathbf{X}}}$ & $\overline{\mathbf{X}}$ & $\mathbf{S E}_{\overline{\mathbf{X}}}$ & $\overline{\mathbf{X}}$ & $\mathbf{S E}_{\overline{\mathbf{X}}}$ \\
\hline \multirow{2}{*}{$\begin{array}{c}\text { Pre Drink } \\
\text { Pre Race }\end{array}$} & ED & 886 & 226.6 & 76 & 16.6 & 394 & 36.0 & $0.04^{\mathrm{A}}$ & 0.004 & $28^{\mathrm{ABC}}$ & 2.4 \\
\hline & PLA & 614 & 180.6 & 58 & 12.6 & 379 & 56.2 & $0.04^{\mathrm{A}}$ & 0.004 & $28^{\mathrm{ABC}}$ & 2.9 \\
\hline \multirow{2}{*}{$\begin{array}{l}\text { Post Drink } \\
\text { Pre Race }\end{array}$} & ED & $2416^{*}$ & 208.5 & 60 & 16.1 & 685 & 84.0 & $0.03^{\mathrm{B}}$ & 0.003 & $27^{\mathrm{AB}}$ & 1.9 \\
\hline & PLA & 604 & 177.3 & 46 & 10.0 & 763 & 108.8 & $0.06^{\mathrm{B}}$ & 0.021 & $26^{\mathrm{AB}}$ & 2.8 \\
\hline \multirow{2}{*}{$6 \mathrm{~km}$} & ED & & & & & & & & & $28^{\mathrm{AB}}$ & 2.5 \\
\hline & PLA & & & & & & & & & $25^{\mathrm{AB}}$ & 2.5 \\
\hline \multirow{2}{*}{17 km } & ED & & & $482 *$ & 29.7 & $2849^{*}$ & 308.7 & & & $31^{\mathrm{AC}}$ & 3.0 \\
\hline & PLA & & & 235 & 19.7 & 2239 & 246.8 & & & $29^{\mathrm{AC}}$ & 2.2 \\
\hline \multirow{2}{*}{$23 \mathrm{~km}$} & ED & & & & & & & & & $34^{\mathrm{D}}$ & 3.0 \\
\hline & PLA & & & & & & & & & $32^{\mathrm{D}}$ & 2.8 \\
\hline \multirow{2}{*}{$29 \mathrm{~km}$} & ED & & & $507 *$ & 46.2 & 2766 & 294.7 & & & $35^{\mathrm{D}}$ & 3.0 \\
\hline & PLA & & & 263 & 21.8 & 2229 & 247.8 & & & $31^{\mathrm{D}}$ & 2.6 \\
\hline \multirow{2}{*}{34 km } & ED & $2615^{*}$ & 216.0 & $628^{*}$ & 73.1 & 2789 & 313.2 & $0.06^{\mathrm{C}}$ & 0.007 & $35^{\mathrm{D}}$ & 2.6 \\
\hline & PLA & 557 & 172.0 & 349 & 66.7 & 2420 & 276.1 & $0.06^{\mathrm{C}}$ & 0.008 & $31^{\mathrm{D}}$ & 2.5 \\
\hline $\begin{array}{r}{ }^{\wedge} \text { Blood } \mathrm{s} \\
*\end{array}$ & $\begin{array}{r}\text { ple was hem } \\
\text { interacti } \\
\text { ractions fou } \\
{ }_{\text {A-D }} \text { Main ef }\end{array}$ & $\begin{array}{l}\text { ysed for } \\
\text { or mai } \\
\text { and LS }\end{array}$ & $\begin{array}{l}\text { ne partic } \\
\text { ffects } \mathrm{u} \\
\text { post-ho } \\
\text { r time v }\end{array}$ & $\begin{array}{l}\text { int at o } \\
\text { seen } \\
\text { reveale } \\
\text { and L }\end{array}$ & $\begin{array}{l}\text { Ime, } r \\
\alpha=.05 \\
\text { fferen }\end{array}$ & $\begin{array}{l}\mathrm{ng} \text { in } \mathrm{N} \\
\text { his assa } \\
\text { etween } \\
\text { evealed }\end{array}$ & $\begin{array}{l}\text { or RM } \\
\text { ess the } \\
\text { ments a } \\
\text { rences }\end{array}$ & $\begin{array}{l}\text { OVA o } \\
\text { le was } 1 \\
\text { times } \\
\text { veen tir }\end{array}$ & $\begin{array}{l}\text { Endorp } \\
\text { oved. } \\
\text { cated }(1 \\
(p<.05)\end{array}$ & $\begin{array}{l}\text { s assay } \\
05) \text {. }\end{array}$ & \\
\hline
\end{tabular}


Table 12: Time to Finish- With Groups Split by Baseline Caffeine Levels (seconds)

\begin{tabular}{|c|c|c|c|c|c|c|}
\hline & \multicolumn{2}{|c|}{ Pooled (N=15) } & \multicolumn{2}{c|}{ Low-Baseline (N=10) } & \multicolumn{2}{c|}{ High-Baseline (N=5) } \\
& $\overline{\mathbf{X}}$ & $\mathbf{S E}_{\overline{\mathbf{X}}}$ & $\overline{\mathbf{X}}$ & $\mathbf{S E}_{\overline{\mathbf{X}}}$ & $\overline{\mathbf{X}}$ & $\mathbf{S E}_{\overline{\mathbf{X}}}$ \\
\hline ED & $3848.5^{*}$ & 78.12 & $3842.9^{*}$ & 89.19 & $3869.3 *$ & 137.53 \\
\hline PLA & 3968.9 & 80.56 & 3965.8 & 100.43 & 3988.0 & 123.84 \\
\hline \multicolumn{7}{|c|}{$*$ Effect found for treatment (p<.05). } \\
\hline
\end{tabular}

Table 13: Blood Caffeine- With Groups Split by Baseline Caffeine Levels (ng/mL)

\begin{tabular}{|c|c|c|c|c|c|c|c|}
\hline \multirow[b]{2}{*}{ Time } & \multirow[b]{2}{*}{ Treatment } & \multicolumn{2}{|c|}{ Pooled $(\mathrm{N}=15)$} & \multicolumn{2}{|c|}{ Low-Baseline $(\mathrm{N}=10)$} & \multicolumn{2}{|c|}{ High-Baseline $(\mathrm{N}=5)$} \\
\hline & & $\overline{\mathbf{X}}$ & $\mathbf{S E}_{\overline{\mathbf{X}}}$ & $\overline{\mathbf{X}}$ & $\mathbf{S E}_{\overline{\mathbf{X}}}$ & $\overline{\mathbf{X}}$ & $\mathbf{S E}_{\overline{\mathbf{X}}}$ \\
\hline \multirow{2}{*}{ Pre Drink Pre Race } & ED & 886 & 226.6 & 379 & 157.2 & 1842 & 266.9 \\
\hline & PLA & 614 & 180.6 & 178 & 72.7 & 1486 & 183.5 \\
\hline \multirow{2}{*}{ Post Drink Pre Race } & ED & $2416^{*}$ & 208.5 & $2081^{*}$ & 236.5 & $3087^{*}$ & 190.5 \\
\hline & PLA & 604 & 177.3 & 148 & 37.9 & 1516 & 108.6 \\
\hline \multirow{2}{*}{34 km } & ED & $2615^{*}$ & 216.0 & $2324 *$ & 245.2 & $3196^{*}$ & 304 \\
\hline & PLA & 557 & 172.0 & 127 & 41.5 & 1418 & 159.2 \\
\hline
\end{tabular}


Table 14: Tap Test- With Groups Split by Baseline Caffeine Levels

\begin{tabular}{|c|c|c|c|c|c|c|c|}
\hline \multirow[b]{2}{*}{ Time } & \multirow[b]{2}{*}{ Treatment } & \multicolumn{2}{|c|}{ Pooled $(\mathrm{N}=15)$} & \multicolumn{2}{|c|}{ Low-Baseline $(\mathrm{N}=10)$} & \multicolumn{2}{|c|}{ High-Baseline $(\mathrm{N}=5)$} \\
\hline & & $\overline{\mathbf{X}}$ & $\mathbf{S E}_{\overline{\mathbf{X}}}$ & $\overline{\mathbf{X}}$ & $\overline{\mathbf{X}}$ & $\mathbf{S E}_{\overline{\mathbf{X}}}$ & $\overline{\mathbf{X}}$ \\
\hline \multirow{2}{*}{ Pre Drink Pre Race } & ED & 3.7 & 0.13 & 3.7 & 0.10 & 3.6 & 0.31 \\
\hline & PLA & 3.7 & 0.11 & 3.7 & 0.09 & 3.6 & 0.26 \\
\hline \multirow{2}{*}{ Post Drink Pre Race } & ED & $3.7^{*}$ & 0.12 & $3.8^{*}$ & 0.09 & 3.8 & 0.29 \\
\hline & PLA & 3.4 & 0.09 & 3.4 & 0.10 & 3.4 & 0.17 \\
\hline \multirow{2}{*}{ Post Race } & ED & $3.9^{*}$ & 0.09 & 4.0 & 0.11 & 4.0 & 0.19 \\
\hline & PLA & 3.8 & 0.11 & 3.8 & 0.14 & 3.7 & 0.19 \\
\hline
\end{tabular}

*Interaction found and LSD post-hocs revealed differences between treatments at the times indicated $(\mathrm{p}<.05)$. 


\section{APPENDIX B: RAW DATA}

Table 7: Participant Characteristics

\begin{tabular}{|c|c|c|c|c|c|c|}
\hline Participant & Age & Gender & Mass (kg) & Height(cm) & $\operatorname{VO} 2 \max (\mathrm{mL} / \mathrm{min})$ & IQ \\
\hline F1 & 25 & $\mathrm{~F}$ & 57.6 & 165.1 & 3160 & 126 \\
\hline F3 & 27 & $\mathrm{~F}$ & 71.9 & 175.26 & 3270 & 109 \\
\hline F4 & 32 & $\mathrm{~F}$ & 62.8 & 165.1 & 3650 & 121 \\
\hline F5 & 41 & $\mathrm{~F}$ & 65.5 & 175.26 & 3430 & 136 \\
\hline F6 & 33 & $\mathrm{~F}$ & 63.2 & 170.18 & 3170 & 109 \\
\hline F8 & 24 & $\mathrm{~F}$ & 77.1 & 175.26 & 3770 & 126 \\
\hline F9 & 27 & $\mathrm{~F}$ & 62 & 175.26 & 3400 & 128 \\
\hline M1 & 43 & $\mathrm{M}$ & 58 & 165.1 & 3610 & 113 \\
\hline M2 & 29 & M & 66.8 & 172.72 & 4690 & 111 \\
\hline M3 & 24 & $\mathrm{M}$ & 60.4 & 172.72 & 3470 & 123 \\
\hline M4 & 32 & $\mathrm{M}$ & 69.9 & 177.8 & 4260 & 114 \\
\hline M7 & 26 & $\mathrm{M}$ & 92 & 185.42 & 5180 & 94 \\
\hline M8 & 29 & $\mathrm{M}$ & 73 & 182.88 & 4620 & 96 \\
\hline M9 & 41 & $\mathrm{M}$ & 66 & 170.18 & 4080 & 133 \\
\hline M10 & 26 & M & 71.5 & 185.42 & 4100 & 114 \\
\hline
\end{tabular}


Table 16: Time to Finish (seconds)

\begin{tabular}{|c|c|c|}
\hline Participant & ED & PLA \\
\hline F1 & 4294.52 & 4338.18 \\
\hline F3 & 4409.25 & 4464.51 \\
\hline F4 & 3933.56 & 4089.37 \\
\hline F5 & 3863.28 & 3905.52 \\
\hline F6 & 4027.61 & 4301.93 \\
\hline F8 & 3917.7 & 4053.71 \\
\hline F9 & 3716.15 & 3878.34 \\
\hline M1 & 3882.18 & 3976.56 \\
\hline M2 & 3461.67 & 3528.71 \\
\hline M3 & 4171.5 & 4356.93 \\
\hline M4 & 3851.34 & 3930.06 \\
\hline M7 & 3372.42 & 3542.76 \\
\hline M8 & 3423.78 & 3432.42 \\
\hline M9 & 3719.34 & 3872.19 \\
\hline M10 & 3682.74 & 3861.6 \\
\hline
\end{tabular}


Table 8: Gas Collection

\begin{tabular}{|c|c|c|c|c|c|c|c|c|c|c|c|c|}
\hline \multirow{3}{*}{ Participant } & \multicolumn{6}{|c|}{$\mathrm{VO}_{2}(\mathrm{~L} / \mathrm{min})$} & \multicolumn{6}{|c|}{$\operatorname{RER}\left(\mathrm{CO}_{2} / \mathrm{VO}_{2}\right)$} \\
\hline & \multicolumn{2}{|c|}{$5-7 \mathrm{~km}$} & \multicolumn{2}{|c|}{$16-18 \mathrm{~km}$} & \multicolumn{2}{|c|}{ 28-30 km } & \multicolumn{2}{|c|}{ 5-7 km } & \multicolumn{2}{|c|}{$16-18 \mathrm{~km}$} & \multicolumn{2}{|c|}{ 28-30 km } \\
\hline & ED & PLA & ED & PLA & ED & PLA & ED & PLA & ED & PLA & ED & PLA \\
\hline F1 & 2.30 & 2.34 & 2.24 & 2.21 & 1.95 & 2.06 & 0.91 & 0.86 & 0.82 & 0.97 & 0.92 & 0.92 \\
\hline F3 & 2.52 & 2.24 & 2.01 & 2.02 & 2.24 & 2.05 & 0.97 & 0.86 & 0.89 & 0.82 & 0.81 & 0.80 \\
\hline F4 & 2.89 & 2.54 & 3.00 & 2.64 & 2.93 & 2.63 & 0.93 & 0.95 & 0.94 & 0.90 & 0.91 & 0.89 \\
\hline F5 & 2.64 & 2.73 & 2.40 & 2.54 & 2.44 & 2.47 & 0.97 & 0.92 & 0.91 & 0.92 & 0.89 & 0.88 \\
\hline F6 & 2.34 & 2.23 & 2.44 & 2.23 & 2.38 & 2.52 & 0.97 & 0.98 & 0.95 & 0.91 & 0.92 & 0.98 \\
\hline F8 & 2.83 & 2.71 & 2.70 & 2.51 & 2.59 & 2.30 & 0.95 & 0.93 & 0.92 & 0.96 & 0.90 & 0.89 \\
\hline F9 & 2.82 & 2.50 & 2.63 & 2.32 & 2.65 & 2.37 & 0.97 & 0.91 & 0.9 & 0.91 & 0.87 & 0.87 \\
\hline M1 & 2.68 & 2.81 & 2.57 & 2.39 & 2.70 & 2.45 & 0.93 & 0.91 & 0.91 & 0.94 & 0.90 & 0.91 \\
\hline M2 & 4.22 & 4.13 & 3.82 & 3.51 & 3.60 & 3.39 & 0.98 & 0.97 & 0.94 & 1.05 & 0.97 & 0.93 \\
\hline M3 & 2.48 & 2.29 & 2.50 & 2.03 & 2.49 & 2.28 & 1.01 & 0.94 & 0.93 & 0.97 & 0.96 & 0.96 \\
\hline M4 & 2.83 & 2.67 & 2.49 & 2.55 & 2.85 & 2.51 & 0.98 & 0.91 & 1.00 & 1.00 & 0.97 & 0.93 \\
\hline M7 & 4.09 & 4.06 & 3.78 & 3.71 & 3.89 & 2.95 & 0.98 & 0.97 & 0.97 & 1.00 & 0.97 & 0.92 \\
\hline M8 & 3.72 & 3.67 & 3.50 & 3.40 & 3.36 & 3.35 & 0.97 & 0.94 & 0.91 & 0.95 & 0.91 & 0.90 \\
\hline M9 & 2.78 & 2.46 & 2.58 & 2.46 & 2.58 & 2.19 & 1.03 & 0.98 & 0.98 & 1.02 & 1.00 & 0.99 \\
\hline M10 & 3.07 & 3.31 & 2.94 & 2.87 & 3.1 & 2.78 & 0.98 & 0.95 & 0.93 & 1.02 & 0.91 & 0.90 \\
\hline
\end{tabular}


Table 9: Heart Rate (beats per minute) at Rest

\begin{tabular}{|c|c|c|c|c|c|c|}
\hline \multirow{2}{*}{ Participant } & \multicolumn{2}{|c|}{$\begin{array}{c}\text { Pre Drink } \\
\text { Pre Race }\end{array}$} & \multicolumn{2}{c|}{$\begin{array}{c}\text { Post Drink } \\
\text { Pre Race }\end{array}$} & \multicolumn{2}{c|}{ End of Visit } \\
& ED & PLA & ED & PLA & ED & PLA \\
\hline F1 & 62 & 63 & 55 & 57 & 65 & 71 \\
\hline F3 & 56 & 59 & 66 & 48 & 62 & 62 \\
\hline F4 & 50 & 61 & 62 & 39 & 84 & 68 \\
\hline F5 & 47 & 47 & 49 & 42 & 63 & 59 \\
\hline F6 & 57 & 57 & 61 & 55 & 70 & 73 \\
\hline F8 & 63 & 64 & 72 & 52 & 77 & 68 \\
\hline F9 & 55 & 59 & 54 & 57 & 65 & 74 \\
\hline M1 & 59 & 51 & 58 & 48 & $\wedge$ & 70 \\
\hline M2 & 55 & 56 & 59 & 57 & 80 & 80 \\
\hline M3 & 70 & 75 & 62 & 62 & 87 & 92 \\
\hline M4 & 57 & 62 & 58 & 58 & 86 & 85 \\
\hline M7 & 52 & 55 & 54 & 51 & 91 & 82 \\
\hline M8 & 49 & 46 & 46 & 41 & 83 & 87 \\
\hline M9 & 54 & 57 & 50 & 56 & 78 & 76 \\
\hline M10 & 67 & 66 & 63 & 68 & 99 & 85 \\
\hline \multicolumn{7}{|c}{$\wedge$ Heart rate not captured by monitors. } \\
\hline
\end{tabular}


Table 10: Heart Rate (beats per minute) $1 \mathrm{~km}$ to $12 \mathrm{~km}$ During the Race

\begin{tabular}{|c|c|c|c|c|c|c|}
\hline \multirow{2}{*}{ Participant } & \multicolumn{2}{|c|}{ 1 km } & \multicolumn{2}{c|}{ 6 km } & \multicolumn{2}{c|}{ 12 km } \\
& ED & PLA & ED & PLA & ED & PLA \\
\hline F1 & 151 & 146 & 168 & 166 & 172 & 157 \\
\hline F3 & 153 & 152 & 159 & 144 & $\wedge$ & 153 \\
\hline F4 & 134 & 126 & 157 & 147 & 173 & 158 \\
\hline F5 & 129 & $\wedge$ & 148 & 140 & 149 & 147 \\
\hline F6 & 144 & 127 & 163 & 149 & 170 & 152 \\
\hline F8 & 163 & 156 & 175 & 170 & 184 & 181 \\
\hline F9 & 152 & 144 & 166 & 157 & 163 & 156 \\
\hline M1 & 126 & 110 & 142 & 142 & 148 & 137 \\
\hline M2 & 167 & 151 & 178 & 175 & 176 & 174 \\
\hline M3 & 135 & 132 & 147 & 145 & 155 & 142 \\
\hline M4 & 149 & 164 & 159 & 155 & 157 & 155 \\
\hline M7 & 165 & 167 & 172 & 173 & 181 & 178 \\
\hline M8 & 163 & 159 & 170 & 166 & 171 & 172 \\
\hline M9 & 160 & 135 & 160 & 147 & 177 & 165 \\
\hline M10 & 133 & 158 & 163 & 177 & 179 & 180 \\
\hline \multicolumn{7}{|l}{ Heart rate not captured by monitors. } \\
\hline
\end{tabular}


Table 20: Heart Rate (beats per minute) $17 \mathrm{~km}$ to $34 \mathrm{~km}$ During the Race

\begin{tabular}{|c|c|c|c|c|c|c|c|c|}
\hline \multirow{2}{*}{ Participant } & \multicolumn{2}{|c|}{$\mathbf{1 7} \mathbf{~ k m}$} & \multicolumn{2}{c|}{ 23 km } & \multicolumn{2}{c|}{ 29 km } & \multicolumn{2}{c|}{ 34 km } \\
& ED & PLA & ED & PLA & ED & PLA & ED & PLA \\
\hline F1 & 168 & 166 & 169 & 160 & 160 & 160 & 178 & 159 \\
\hline F3 & 141 & 137 & 145 & 135 & 147 & 137 & 145 & 150 \\
\hline F4 & 167 & 166 & 173 & 138 & 177 & $\wedge$ & 180 & 132 \\
\hline F5 & 140 & 141 & 145 & 148 & 143 & 137 & 153 & 143 \\
\hline F6 & 168 & 159 & 167 & 160 & 168 & 167 & 166 & 164 \\
\hline F8 & 179 & 169 & 178 & 172 & 174 & 156 & 178 & 160 \\
\hline F9 & 160 & 149 & 161 & 149 & 161 & 151 & 168 & $\wedge$ \\
\hline M1 & 146 & 132 & 143 & 143 & 149 & 137 & 160 & 149 \\
\hline M2 & 178 & 169 & 174 & 166 & 175 & 165 & 176 & 171 \\
\hline M3 & 155 & 140 & 156 & 144 & 154 & 150 & 154 & 161 \\
\hline M4 & 151 & 160 & 152 & 146 & 165 & 163 & 159 & 159 \\
\hline M7 & 172 & 176 & 175 & 170 & 178 & 156 & 179 & 173 \\
\hline M8 & 168 & 166 & 171 & 170 & 166 & 164 & 171 & 168 \\
\hline M9 & 163 & 150 & 174 & 153 & 165 & 148 & 177 & 155 \\
\hline M10 & 160 & 174 & 172 & 175 & 172 & 170 & 181 & 176 \\
\hline \multicolumn{7}{|c|}{$\wedge$ Heart rate not captured by monitors. } & & \\
\hline
\end{tabular}


Table 11: Heart Rate (beats per minute) During the Stroop Test After the Race

\begin{tabular}{|c|c|c|c|c|c|c|c|c|c|c|}
\hline \multirow{2}{*}{ Participant } & \multicolumn{2}{|c|}{ Start } & \multicolumn{2}{c|}{ 30 seconds } & \multicolumn{2}{c|}{ 60 seconds } & \multicolumn{2}{c|}{ 90 seconds } & \multicolumn{2}{c|}{ 120 seconds } \\
& ED & PLA & ED & PLA & ED & ED & ED & PLA & ED & PLA \\
\hline F1 & 170 & 157 & 1364 & 164 & 164 & 160 & 164 & 161 & 162 & 162 \\
\hline F3 & 129 & 130 & 133 & 138 & 139 & 135 & 137 & 137 & 132 & 137 \\
\hline F4 & 180 & $\wedge$ & 171 & $\wedge$ & $\wedge$ & $\wedge$ & 153 & 130 & 144 & 130 \\
\hline F5 & 147 & 135 & 137 & 130 & 136 & 130 & 130 & 132 & 132 & 133 \\
\hline F6 & 163 & 151 & 158 & 146 & 161 & 154 & 161 & 157 & 164 & 154 \\
\hline F8 & 161 & 150 & 168 & 143 & 166 & 156 & 165 & 158 & 164 & 156 \\
\hline F9 & 168 & $\wedge$ & 156 & $\wedge$ & 147 & $\wedge$ & 142 & $\wedge$ & 142 & $\wedge$ \\
\hline M1 & 141 & $\wedge$ & 136 & $\wedge$ & 137 & $\wedge$ & 137 & $\wedge$ & 135 & $\wedge$ \\
\hline M2 & 159 & 169 & 157 & 160 & 157 & 157 & 161 & 159 & 156 & 159 \\
\hline M3 & 159 & 164 & 154 & 160 & 150 & 160 & 149 & 155 & 148 & 150 \\
\hline M4 & 160 & 158 & 155 & 152 & 154 & 157 & 155 & 159 & 161 & 160 \\
\hline M7 & 189 & 186 & 181 & 176 & 173 & 174 & 173 & 173 & 174 & 174 \\
\hline M8 & 176 & 172 & 165 & 161 & 164 & 162 & 165 & 162 & 158 & 162 \\
\hline M9 & 160 & 160 & 155 & 154 & 149 & 154 & 160 & 152 & 164 & 161 \\
\hline M10 & 177 & 158 & 172 & 162 & 176 & 167 & 175 & 173 & 174 & 172 \\
\hline \multicolumn{3}{|c|}{$\wedge$ Heart rate not captured by monitors. } & & & \\
\hline
\end{tabular}


Table 12: RPE (Borg 6-20)

\begin{tabular}{|c|c|c|c|c|c|c|c|c|c|c|c|c|c|c|}
\hline \multirow{2}{*}{ Participant } & \multicolumn{2}{|c|}{$1 \mathrm{~km}$} & \multicolumn{2}{|c|}{$6 \mathrm{~km}$} & \multicolumn{2}{|c|}{12 km } & \multicolumn{2}{|c|}{17 km } & \multicolumn{2}{|c|}{$23 \mathrm{~km}$} & \multicolumn{2}{|c|}{$29 \mathrm{~km}$} & \multicolumn{2}{|c|}{$34 \mathrm{~km}$} \\
\hline & ED & PLA & ED & PLA & ED & PLA & ED & PLA & ED & PLA & ED & PLA & ED & PLA \\
\hline F1 & 12 & 12 & 14 & 13 & 15 & 15 & 15 & 15 & 16 & 16 & 15 & 15 & 17 & 17 \\
\hline F3 & 11 & 10 & 13 & 14 & 15 & 15 & 14 & 15 & 15 & 17 & 15 & 15 & 16 & 16 \\
\hline F4 & 7 & 7 & 12 & 13 & 14 & 13 & 13 & 13 & 15 & 14 & 14 & 13 & 14 & 15 \\
\hline F5 & 12 & 12 & 13 & 13 & 15 & 14 & 13 & 13 & 14 & 15 & 13 & 13 & 16 & 15 \\
\hline F6 & 12 & 11 & 15 & 14 & 17 & 15 & 17 & 16 & 17 & 17 & 16 & 17 & 18 & 17 \\
\hline F8 & 13 & 12 & 15 & 14 & 16 & 16 & 16 & 15 & 16 & 17 & 16 & 16 & 17 & 17 \\
\hline F9 & 13 & 13 & 13 & 14 & 15 & 14 & 13 & 14 & 14 & 13 & 14 & 14 & 17 & 16 \\
\hline M1 & 13 & 13 & 15 & 15 & 16 & 16 & 16 & 15 & 15 & 17 & 17 & 17 & 17 & 17 \\
\hline M2 & 14 & 13 & 16 & 15 & 17 & 16 & 17 & 16 & 17 & 15 & 17 & 17 & 18 & 18 \\
\hline M3 & 13 & 14 & 14 & 14 & 15 & 15 & 15 & 15 & 16 & 16 & 16 & 17 & 17 & 17 \\
\hline M4 & 10 & 12 & 13 & 14 & 15 & 14 & 15 & 15 & 15 & 15 & 15 & 15 & 15 & 15 \\
\hline M7 & 13 & 14 & 15 & 15 & 17 & 17 & 17 & 17 & 19 & 18 & 17 & 15 & 19 & 19 \\
\hline M8 & 12 & 13 & 13 & 15 & 16 & 17 & 14 & 15 & 17 & 18 & 16 & 18 & 17 & 19 \\
\hline M9 & 12 & 13 & 14 & 14 & 15 & 15 & 14 & 15 & 16 & 16 & 15 & 16 & 17 & 16 \\
\hline M10 & 9 & 15 & 15 & 19 & 19 & 20 & 17 & 20 & 19 & 20 & 19 & 20 & 20 & 20 \\
\hline
\end{tabular}


Table 13: Executive Function Taps per Second

\begin{tabular}{|c|c|c|c|c|c|c|}
\hline \multirow{2}{*}{ Participant } & \multicolumn{2}{|c|}{$\begin{array}{c}\text { Pre Drink Pre } \\
\text { Race }\end{array}$} & \multicolumn{2}{c|}{$\begin{array}{c}\text { Post Drink Pre } \\
\text { Race }\end{array}$} & \multicolumn{2}{c|}{ Post Race } \\
& ED & PLA & ED & PLA & ED & PLA \\
\hline F1 & 5.4 & 5.1 & 5.3 & 5.1 & 5.9 & 5.4 \\
\hline F3 & 4.6 & 4.9 & 5.1 & 4.8 & 5.1 & 4.6 \\
\hline F4 & 4.9 & 4.8 & 4.7 & 4.7 & 5.4 & 5.6 \\
\hline F5 & 5.5 & 4.0 & 4.4 & 5.0 & 5.4 & 5.2 \\
\hline F6 & 6.1 & 6.1 & 6.2 & 4.7 & 6.2 & 6.5 \\
\hline F8 & 6.5 & 6.1 & 6.8 & 5.9 & 7.2 & 6.4 \\
\hline F9 & 4.6 & 4.6 & 4.9 & 4.2 & 5.0 & 4.6 \\
\hline M1 & 5.9 & 5.5 & 6.2 & 5.4 & 6.3 & 5.9 \\
\hline M2 & 6.3 & 6.0 & 6.1 & 6.1 & 6.9 & 6.8 \\
\hline M3 & 6.6 & 6.9 & 7.0 & 6.6 & 6.2 & 5.9 \\
\hline M4 & 5.5 & 5.6 & 5.9 & 5.6 & 6.2 & 6.0 \\
\hline M7 & 6.7 & 6.5 & 6.9 & 6.3 & 7.1 & 6.7 \\
\hline M8 & 6.3 & 5.7 & 6.1 & 5.6 & 6.7 & 6.1 \\
\hline M9 & 6.3 & 5.8 & 6.3 & 5.8 & 6.4 & 6.1 \\
\hline M10 & 6.1 & 6.0 & 6.0 & 5.8 & 6.4 & 6.1 \\
\hline
\end{tabular}


Table 14: Executive Function Reaction Time (milliseconds)

\begin{tabular}{|c|c|c|c|c|c|c|}
\hline \multirow{2}{*}{ Participant } & \multicolumn{2}{|c|}{$\begin{array}{c}\text { Pre Drink Pre } \\
\text { Race }\end{array}$} & \multicolumn{2}{c|}{$\begin{array}{c}c \mid \\
\text { Race }\end{array}$} & \multicolumn{2}{c|}{ Post Race } \\
& ED & PLA & ED & PLA & ED & PLA \\
\hline F1 & 560 & 534 & 685 & 565 & 570 & 590 \\
\hline F3 & 482 & 407 & 425 & 446 & 509 & 466 \\
\hline F4 & 621 & 515 & 594 & 528 & 486 & 427 \\
\hline F5 & 506 & 643 & 544 & 412 & 426 & 451 \\
\hline F6 & 499 & 602 & 504 & 550 & 567 & 530 \\
\hline F8 & 472 & 451 & 459 & 493 & 459 & 565 \\
\hline F9 & 627 & 827 & 561 & 509 & 693 & 887 \\
\hline M1 & 646 & 598 & 499 & 538 & 570 & 616 \\
\hline M2 & 606 & 675 & 766 & 627 & 478 & 685 \\
\hline M3 & 504 & 525 & 604 & 648 & 622 & 590 \\
\hline M4 & 636 & 571 & 546 & 514 & 452 & 463 \\
\hline M7 & 473 & 533 & 473 & 413 & 429 & 474 \\
\hline M8 & 447 & 484 & 541 & 409 & 401 & 403 \\
\hline M9 & 481 & 465 & 530 & 613 & 436 & 522 \\
\hline M10 & 462 & 512 & 632 & 560 & 508 & 458 \\
\hline
\end{tabular}


Table 15: Executive Function Movement Time (milliseconds)

\begin{tabular}{|c|c|c|c|c|c|c|}
\hline \multirow{2}{*}{ Participant } & \multicolumn{2}{|c|}{$\begin{array}{c}\text { Pre Drink Pre } \\
\text { Race }\end{array}$} & \multicolumn{2}{c|}{$\begin{array}{c}\text { Post Drink Pre } \\
\text { Race }\end{array}$} & \multicolumn{2}{c|}{ Post Race } \\
& ED & PLA & ED & PLA & ED & PLA \\
\hline F1 & 301 & 358 & 344 & 378 & 316 & 340 \\
\hline F3 & 256 & 248 & 230 & 241 & 220 & 199 \\
\hline F4 & 439 & 447 & 469 & 468 & 348 & 426 \\
\hline F5 & 373 & 429 & 370 & 325 & 374 & 344 \\
\hline F6 & 332 & 344 & 307 & 291 & 328 & 305 \\
\hline F8 & 234 & 224 & 235 & 223 & 189 & 231 \\
\hline F9 & 468 & 501 & 433 & 458 & 376 & 465 \\
\hline M1 & 550 & 542 & 481 & 491 & 483 & 545 \\
\hline M2 & 449 & 473 & 453 & 496 & 478 & 494 \\
\hline M3 & 308 & 279 & 278 & 307 & 297 & 279 \\
\hline M4 & 346 & 387 & 376 & 385 & 347 & 377 \\
\hline M7 & 339 & 330 & 333 & 352 & 290 & 359 \\
\hline M8 & 259 & 222 & 232 & 224 & 217 & 211 \\
\hline M9 & 221 & 160 & 190 & 213 & 220 & 204 \\
\hline M10 & 326 & 274 & 313 & 322 & 308 & 269 \\
\hline
\end{tabular}


Table 16: Executive Function Total Time (milliseconds)

\begin{tabular}{|c|c|c|c|c|c|c|}
\hline \multirow{2}{*}{ Participant } & \multicolumn{2}{|c|}{$\begin{array}{c}\text { Pre Drink Pre } \\
\text { Race }\end{array}$} & \multicolumn{2}{c|}{$\begin{array}{c}\text { Post Drink Pre } \\
\text { Race }\end{array}$} & \multicolumn{2}{c|}{ Post Race } \\
& ED & PLA & ED & PLA & ED & PLA \\
\hline F1 & 861 & 892 & 909 & 1063 & 887 & 930 \\
\hline F3 & 738 & 655 & 676 & 665 & 729 & 665 \\
\hline F4 & 1060 & 962 & 997 & 1062 & 835 & 853 \\
\hline F5 & 879 & 1072 & 783 & 869 & 800 & 794 \\
\hline F6 & 831 & 946 & 857 & 795 & 894 & 835 \\
\hline F8 & 705 & 676 & 729 & 682 & 648 & 796 \\
\hline F9 & 1095 & 1328 & 942 & 1019 & 1069 & 1352 \\
\hline M1 & 1196 & 1140 & 1019 & 991 & 1053 & 1161 \\
\hline M2 & 1055 & 1149 & 1081 & 1262 & 956 & 1179 \\
\hline M3 & 812 & 804 & 926 & 910 & 918 & 869 \\
\hline M4 & 982 & 958 & 890 & 931 & 799 & 840 \\
\hline M7 & 812 & 863 & 746 & 825 & 719 & 833 \\
\hline M8 & 705 & 706 & 640 & 765 & 618 & 614 \\
\hline M9 & 702 & 624 & 803 & 743 & 656 & 726 \\
\hline M10 & 787 & 786 & 873 & 954 & 816 & 728 \\
\hline
\end{tabular}


Table 17: Tap Test Percent of Precise Responses

\begin{tabular}{|c|c|c|c|c|c|c|}
\hline \multirow{2}{*}{ Participant } & \multicolumn{2}{|c|}{ Pre Drink Pre Race } & \multicolumn{2}{c|}{ Post Drink Pre Race } & \multicolumn{2}{c|}{ Post Race } \\
& ED & PLA & ED & PLA & ED & PLA \\
\hline F1 & 99.6 & 99.7 & 99.6 & 99.6 & 99.7 & 100.0 \\
\hline F3 & $\wedge$ & $\wedge$ & $\wedge$ & $\wedge$ & $\wedge$ & $\wedge$ \\
\hline F4 & $\wedge$ & $\wedge$ & $\wedge$ & $\wedge$ & $\wedge$ & $\wedge$ \\
\hline F5 & 99.3 & 100.0 & 100.0 & 99.6 & 100.0 & 100.0 \\
\hline F6 & 100.0 & 100.0 & 100.0 & 100.0 & 100.0 & 100.0 \\
\hline F8 & 100.0 & 98.3 & 97.5 & 99.6 & 95.7 & 97.4 \\
\hline F9 & 99.6 & 100.0 & 99.6 & 99.4 & 100.0 & 99.6 \\
\hline M1 & $\wedge$ & $\wedge$ & $\wedge$ & $\wedge$ & $\wedge$ & $\wedge$ \\
\hline M2 & $\wedge$ & $\wedge$ & $\wedge$ & $\wedge$ & $\wedge$ & $\wedge$ \\
\hline M3 & 100.0 & 100.0 & 99.7 & 100.0 & 99.7 & 100.0 \\
\hline M4 & 99.7 & 100.0 & 99.7 & 99.6 & 100.0 & 100.0 \\
\hline M7 & 100.0 & 100.0 & 99.0 & 99.0 & 97.6 & 98.4 \\
\hline M8 & 100.0 & 100.0 & 100.0 & 100.0 & 99.7 & 100.0 \\
\hline M9 & 100.0 & 100.0 & 100.0 & 100.0 & 100.0 & 100.0 \\
\hline M10 & 100.0 & 100.0 & 99.2 & 99.2 & 99.3 & 99.6 \\
\hline
\end{tabular}


Table 18: Tap Test Taps per Second

\begin{tabular}{|c|c|c|c|c|c|c|}
\hline \multirow{2}{*}{ Participant } & \multicolumn{2}{|c|}{ Pre Drink Pre Race } & \multicolumn{2}{c|}{ Post Drink Pre Race } & \multicolumn{2}{c|}{ Post Race } \\
& ED & PLA & ED & PLA & ED & PLA \\
\hline F1 & 3.5 & 3.6 & 3.4 & 3.2 & 3.5 & 3.5 \\
\hline F3 & 3.4 & $3.3^{\wedge}$ & 3.6 & 3.1 & 3.4 & 3.2 \\
\hline F4 & 2.3 & 2.5 & 2.6 & 2.8 & 3.6 & 3.2 \\
\hline F5 & 3.7 & 3.8 & 3.5 & 3.4 & 3.7 & 3.8 \\
\hline F6 & 3.9 & 3.8 & 3.8 & 3.6 & 4.0 & 4.1 \\
\hline F8 & 4.0 & 4.2 & 4.3 & 3.4 & 4.7 & 4.3 \\
\hline F9 & 3.0 & 3.1 & 3.3 & 2.9 & 3.5 & 3.1 \\
\hline M1 & 3.5 & 3.4 & 3.7 & 3.1 & 4.2 & 3.5 \\
\hline M2 & 3.9 & 3.8 & 3.7 & 3.2 & 4.0 & 4.4 \\
\hline M3 & 4.1 & 3.9 & 4.0 & 3.9 & 3.8 & 3.6 \\
\hline M4 & 3.6 & 3.7 & 3.8 & 3.7 & 4.0 & 3.8 \\
\hline M7 & 4.1 & 4.1 & 4.5 & 4.0 & 4.1 & 3.6 \\
\hline M8 & 4.0 & 3.7 & 4.0 & 3.7 & 4.3 & 4.3 \\
\hline M9 & 4.4 & 3.9 & 4.3 & 3.5 & 4.3 & 4.2 \\
\hline M10 & 3.8 & 3.7 & 3.8 & 3.3 & 4.0 & 3.8 \\
\hline Data file was saved over but data point estimated using method described in Appendix F. \\
\hline
\end{tabular}


Table 19: Stroop Test "Read" Stimuli

\begin{tabular}{|c|c|c|c|c|c|c|c|c|}
\hline & \multicolumn{3}{|c|}{ Percent of Correct Responses } & \multicolumn{3}{c|}{ Reaction Time (seconds) } \\
Participant & Pre Drink Pre Race & \multicolumn{2}{c|}{ Post Race } & \multicolumn{2}{c|}{$\begin{array}{c}\text { Pre Drink Pre } \\
\text { Race }\end{array}$} & \multicolumn{2}{c|}{ Post Race } \\
& ED & PLA & ED & PLA & ED & PLA & ED & PLA \\
\hline F1 & 100.0 & 100.0 & 85.7 & 100.0 & 0.90 & 0.92 & 0.77 & 0.94 \\
\hline F3 & 100.0 & 100.0 & 100.0 & 100.0 & 1.11 & 0.96 & 1.05 & 0.80 \\
\hline F4 & 60.0 & 0.0 & 90.9 & 28.6 & 2.11 & 0.00 & 1.50 & 1.12 \\
\hline F5 & 91.7 & 100.0 & 100.0 & 100.0 & 1.17 & 1.20 & 1.39 & 1.25 \\
\hline F6 & 100.0 & 100.0 & 100.0 & 100.0 & 0.91 & 1.03 & 0.91 & 0.89 \\
\hline F8 & 100.0 & 100.0 & 100.0 & 100.0 & 0.89 & 0.88 & 0.89 & 0.77 \\
\hline F9 & 100.0 & 100.0 & 100.0 & 100.0 & 1.01 & 0.96 & 1.09 & 1.03 \\
\hline M1 & 66.7 & 100.0 & 90.0 & 100.0 & 1.23 & 1.94 & 1.33 & 1.39 \\
\hline M2 & 100.0 & 100.0 & 100.0 & 100.0 & 1.40 & 1.50 & 1.30 & 1.25 \\
\hline M3 & 100.0 & 100.0 & 88.9 & 100.0 & 0.89 & 0.96 & 0.86 & 0.87 \\
\hline M4 & 90.9 & 100.0 & 100.0 & 100.0 & 1.72 & 1.52 & 1.21 & 1.47 \\
\hline M7 & 100.0 & 85.7 & 92.3 & 90.0 & 1.13 & 1.66 & 1.44 & 1.60 \\
\hline M8 & 100.0 & 100.0 & 100.0 & 100.0 & 1.19 & 1.14 & 0.96 & 1.08 \\
\hline M9 & 90.9 & 92.9 & 60.0 & 80.0 & 0.94 & 0.82 & 0.84 & 0.80 \\
\hline M10 & 85.7 & 83.3 & 81.8 & 85.7 & 1.07 & 1.38 & 0.88 & 0.78 \\
\hline
\end{tabular}


Table 20: Stroop Test "Incongruent" Stimuli

\begin{tabular}{|c|c|c|c|c|c|c|c|c|}
\hline & \multicolumn{3}{|c|}{ Percent of Correct Responses } & \multicolumn{3}{c|}{ Reaction Time (seconds) } \\
Participant & Pre Drink Pre Race & \multicolumn{2}{c|}{ Post Race } & \multicolumn{2}{c|}{$\begin{array}{c}\text { Pre Drink Pre } \\
\text { Race }\end{array}$} & \multicolumn{2}{c|}{ Post Race } \\
& ED & PLA & ED & PLA & ED & PLA & ED & PLA \\
\hline F1 & 100.0 & 100.0 & 100.0 & 100.0 & 0.76 & 0.85 & 0.77 & 0.85 \\
\hline F3 & 95.2 & 100.0 & 85.0 & 88.9 & 0.96 & 0.96 & 0.81 & 0.94 \\
\hline F4 & 100.0 & 95.7 & 100.0 & 100.0 & 1.01 & 1.15 & 0.92 & 1.05 \\
\hline F5 & 100.0 & 100.0 & 100.0 & 100.0 & 1.13 & 1.31 & 1.33 & 1.23 \\
\hline F6 & 100.0 & 100.0 & 100.0 & 100.0 & 1.09 & 1.21 & 1.06 & 1.09 \\
\hline F8 & 95.5 & 100.0 & 90.5 & 95.7 & 0.95 & 0.90 & 0.88 & 0.75 \\
\hline F9 & 96.0 & 100.0 & 100.0 & 100.0 & 0.99 & 0.92 & 0.90 & 0.90 \\
\hline M1 & 100.0 & 91.7 & 92.3 & 100.0 & 1.84 & 1.40 & 1.51 & 1.32 \\
\hline M2 & 100.0 & 100.0 & 100.0 & 94.7 & 1.37 & 1.26 & 1.28 & 1.13 \\
\hline M3 & 100.0 & 100.0 & 90.0 & 100.0 & 0.77 & 0.83 & 0.82 & 0.85 \\
\hline M4 & 100.0 & 100.0 & 100.0 & 100.0 & 1.41 & 1.54 & 1.18 & 1.36 \\
\hline M7 & 95.5 & 100.0 & 100.0 & 92.9 & 1.01 & 1.50 & 1.05 & 1.30 \\
\hline M8 & 100.0 & 100.0 & 100.0 & 94.1 & 1.22 & 1.01 & 0.87 & 0.92 \\
\hline M9 & 100.0 & 93.3 & 100.0 & 95.0 & 0.95 & 0.88 & 0.78 & 0.80 \\
\hline M10 & 94.7 & 90.0 & 94.7 & 80.0 & 1.06 & 0.99 & 0.73 & 0.96 \\
\hline
\end{tabular}


Table 21: Stroop Test Total (Both Stimuli Types)

\begin{tabular}{|c|c|c|c|c|c|c|c|c|}
\hline \multirow{2}{*}{ Participant } & \multicolumn{3}{|c|}{ Percent of Correct Responses } & \multicolumn{3}{c|}{ Reaction Time (seconds) } \\
& Pre Drink Pre Race & \multicolumn{2}{c|}{ Post Race } & \multicolumn{2}{c|}{ Pre Drink Pre Race } & \multicolumn{2}{c|}{ Post Race } \\
& ED & PLA & ED & PLA & ED & PLA & ED & PLA \\
\hline F1 & 100.0 & 100.0 & 96.8 & 100.0 & 0.81 & 0.87 & 0.77 & 0.87 \\
\hline F3 & 96.4 & 100.0 & 89.7 & 92.9 & 0.99 & 0.96 & 0.88 & 0.89 \\
\hline F4 & 81.8 & 91.7 & 96.2 & 77.3 & 1.51 & 1.10 & 1.16 & 1.07 \\
\hline F5 & 96.2 & 100.0 & 100.0 & 100.0 & 1.15 & 1.29 & 1.35 & 1.24 \\
\hline F6 & 100.0 & 100.0 & 100.0 & 100.0 & 1.05 & 1.16 & 1.01 & 0.99 \\
\hline F8 & 96.4 & 100.0 & 93.1 & 96.8 & 0.94 & 0.89 & 0.88 & 0.76 \\
\hline F9 & 96.4 & 100.0 & 100.0 & 100.0 & 0.99 & 0.93 & 0.93 & 0.91 \\
\hline M1 & 90.0 & 95.2 & 91.3 & 100.0 & 1.66 & 1.63 & 1.43 & 1.35 \\
\hline M2 & 100.0 & 100.0 & 100.0 & 96.2 & 1.38 & 1.37 & 1.29 & 1.16 \\
\hline M3 & 100.0 & 100.0 & 89.7 & 100.0 & 0.78 & 0.86 & 0.83 & 0.86 \\
\hline M4 & 95.5 & 100.0 & 100.0 & 100.0 & 1.56 & 1.53 & 1.19 & 1.41 \\
\hline M8 & 96.4 & 95.5 & 96.0 & 91.7 & 1.04 & 1.55 & 1.25 & 1.42 \\
\hline M9 & 100.0 & 100.0 & 100.0 & 96.4 & 1.20 & 1.06 & 0.89 & 0.98 \\
\hline M10 & 96.4 & 93.1 & 86.2 & 90.0 & 0.94 & 0.85 & 0.80 & 0.80 \\
\hline
\end{tabular}


Table 22: Reaction Time Test Percent Outer Rim Responses

\begin{tabular}{|c|c|c|c|c|c|c|}
\hline \multirow{2}{*}{ Participant } & \multicolumn{2}{|c|}{$\begin{array}{c}\text { Pre Drink Pre } \\
\text { Race }\end{array}$} & \multicolumn{2}{c|}{$\begin{array}{c}\text { Post drink Pre } \\
\text { Race }\end{array}$} & \multicolumn{2}{c|}{ Post Race } \\
& ED & PLA & ED & PLA & ED & PLA \\
\hline F1 & 18.8 & 18.8 & 43.8 & 37.5 & 56.3 & 25.0 \\
\hline F3 & 62.5 & 81.3 & 75.0 & 68.8 & 43.8 & 87.5 \\
\hline F4 & 23.1 & 15.4 & 0.0 & 0.0 & 15.4 & 0.0 \\
\hline F5 & 68.8 & 50.0 & 81.3 & 25.0 & 68.8 & 43.8 \\
\hline F6 & 25.0 & 25.0 & 41.7 & 66.7 & 25.0 & 33.3 \\
\hline F8 & 66.7 & 53.3 & 73.3 & 60.0 & 66.7 & 93.3 \\
\hline F9 & 12.5 & 25.0 & 31.3 & 31.3 & 56.3 & 18.8 \\
\hline M1 & 40.0 & 20.0 & 0.0 & 26.7 & 13.3 & 26.7 \\
\hline M2 & 0.0 & 0.0 & 9.1 & 0.0 & 9.1 & 9.1 \\
\hline M3 & 56.3 & 43.8 & 56.3 & 50.0 & 43.8 & 50.0 \\
\hline M4 & 0.0 & 26.7 & 6.7 & 13.3 & 13.3 & 26.7 \\
\hline M7 & 12.5 & 31.3 & 31.3 & 56.3 & 37.5 & 56.3 \\
\hline M8 & 27.3 & 27.3 & 54.6 & 45.5 & 54.6 & 45.5 \\
\hline M9 & 78.6 & 92.9 & 85.7 & 85.7 & 92.9 & 64.3 \\
\hline M10 & 37.5 & 62.5 & 31.3 & 68.8 & 37.5 & 37.5 \\
\hline
\end{tabular}


Table 23: Reaction Time Test Percent Correct and Precise

\begin{tabular}{|c|c|c|c|c|c|c|}
\hline \multirow{2}{*}{ Participant } & \multicolumn{2}{|c|}{ Pre Drink Pre } & \multicolumn{2}{c|}{$\begin{array}{c}\text { Post drink Pre } \\
\text { Race }\end{array}$} & \multicolumn{2}{c|}{ Post Race } \\
& ED & PLA & ED & PLA & ED & PLA \\
\hline F1 & 81.3 & 81.3 & 56.3 & 62.5 & 43.8 & 68.8 \\
\hline F3 & 37.5 & 18.8 & 25.0 & 31.3 & 43.8 & 12.5 \\
\hline F4 & 76.9 & 76.9 & 100.0 & 100.0 & 84.6 & 100.0 \\
\hline F5 & 31.3 & 50.0 & 18.8 & 75.0 & 31.3 & 56.3 \\
\hline F6 & 75.0 & 66.7 & 58.3 & 33.3 & 75.0 & 66.7 \\
\hline F8 & 33.3 & 46.7 & 26.7 & 33.3 & 33.3 & 6.7 \\
\hline F9 & 87.5 & 75.0 & 62.5 & 68.8 & 43.8 & 75.0 \\
\hline M1 & 53.3 & 80.0 & 100.0 & 73.3 & 86.7 & 73.3 \\
\hline M2 & 90.9 & 100.0 & 90.9 & 100.0 & 90.9 & 90.9 \\
\hline M3 & 43.8 & 56.3 & 43.8 & 50.0 & 56.3 & 50.0 \\
\hline M4 & 100.0 & 73.3 & 93.3 & 80.0 & 86.7 & 66.7 \\
\hline M7 & 87.5 & 68.8 & 68.8 & 43.8 & 62.5 & 43.8 \\
\hline M8 & 72.7 & 72.7 & 45.5 & 45.5 & 27.3 & 54.6 \\
\hline M9 & 21.4 & 7.1 & 14.3 & 14.3 & 7.1 & 28.6 \\
\hline M10 & 62.5 & 37.5 & 68.8 & 31.3 & 62.5 & 62.5 \\
\hline
\end{tabular}


Table 24: Reaction Time Test Reaction Time when Correct (milliseconds)

\begin{tabular}{|c|c|c|c|c|c|c|}
\hline \multirow{2}{*}{ Participant } & \multicolumn{2}{|c|}{ Pre Drink Pre Race } & \multicolumn{2}{c|}{ Post drink Pre Race } & \multicolumn{2}{c|}{ Post Race } \\
& ED & PLA & ED & PLA & ED & PLA \\
\hline F1 & 356 & 392 & 380 & 486 & 333 & 362 \\
\hline F3 & 449 & 376 & 461 & 423 & 504 & 413 \\
\hline F4 & 463 & 385 & 420 & 361 & 423 & 453 \\
\hline F5 & 447 & 384 & 334 & 414 & 373 & 381 \\
\hline F6 & 460 & 524 & 391 & 411 & 377 & 613 \\
\hline F8 & 425 & 304 & 373 & 409 & 350 & 351 \\
\hline F9 & 325 & 389 & 390 & 470 & 347 & 330 \\
\hline M1 & 585 & 516 & 475 & 601 & 507 & 423 \\
\hline M3 & 341 & 344 & 396 & 349 & 387 & 773 \\
\hline M4 & 527 & 534 & 507 & 500 & 528 & 571 \\
\hline M7 & 356 & 358 & 325 & 490 & 354 & 312 \\
\hline M8 & 471 & 378 & 327 & 327 & 364 & 460 \\
\hline M9 & 458 & 340 & 356 & 408 & 413 & 401 \\
\hline M10 & 368 & 576 & 454 & 440 & 466 & 422 \\
\hline
\end{tabular}


Table 25: Reaction Time Test Movement Time when Correct (milliseconds)

\begin{tabular}{|c|c|c|c|c|c|c|}
\hline \multirow{2}{*}{ Participant } & \multicolumn{2}{|c|}{ Pre Drink Pre Race } & \multicolumn{2}{c|}{ Post drink Pre Race } & \multicolumn{2}{c|}{ Post Race } \\
& ED & PLA & ED & PLA & ED & PLA \\
\hline F1 & 318 & 358 & 302 & 337 & 283 & 338 \\
\hline F3 & 279 & 209 & 237 & 213 & 232 & 187 \\
\hline F4 & 508 & 580 & 503 & 568 & 474 & 480 \\
\hline F5 & 330 & 338 & 333 & 344 & 329 & 302 \\
\hline F6 & 347 & 330 & 298 & 294 & 310 & 303 \\
\hline F8 & 248 & 234 & 226 & 186 & 190 & 190 \\
\hline F9 & 501 & 477 & 376 & 411 & 422 & 448 \\
\hline M1 & 355 & 340 & 406 & 383 & 316 & 328 \\
\hline M2 & 544 & 633 & 612 & 590 & 558 & 552 \\
\hline M4 & 256 & 270 & 237 & 237 & 210 & 220 \\
\hline M7 & 595 & 548 & 520 & 460 & 466 & 393 \\
\hline M8 & 319 & 476 & 462 & 446 & 403 & 429 \\
\hline M9 & 192 & 289 & 234 & 268 & 132 & 234 \\
\hline M10 & 345 & 165 & 174 & 192 & 175 & 167 \\
\hline
\end{tabular}


Table 26: Reaction Time Test Total Time when Correct (milliseconds)

\begin{tabular}{|c|c|c|c|c|c|c|}
\hline \multirow{2}{*}{ Participant } & \multicolumn{2}{|c|}{ Pre Drink Pre Race } & \multicolumn{2}{c|}{ Post drink Pre Race } & \multicolumn{2}{c|}{ Post Race } \\
& ED & PLA & ED & PLA & ED & PLA \\
\hline F1 & 673 & 750 & 682 & 823 & 616 & 699 \\
\hline F3 & 728 & 584 & 698 & 636 & 736 & 600 \\
\hline F4 & 972 & 966 & 923 & 928 & 898 & 932 \\
\hline F5 & 777 & 722 & 667 & 758 & 702 & 683 \\
\hline F6 & 807 & 854 & 689 & 705 & 687 & 916 \\
\hline F8 & 673 & 538 & 599 & 595 & 540 & 541 \\
\hline F9 & 826 & 866 & 766 & 880 & 770 & 778 \\
\hline M1 & 940 & 856 & 881 & 984 & 823 & 750 \\
\hline M2 & 885 & 976 & 1008 & 939 & 945 & 1325 \\
\hline M3 & 782 & 804 & 744 & 738 & 738 & 790 \\
\hline M4 & 951 & 906 & 845 & 950 & 820 & 705 \\
\hline M7 & 815 & 854 & 789 & 772 & 766 & 889 \\
\hline M8 & 789 & 629 & 589 & 675 & 545 & 635 \\
\hline M9 & 650 & 741 & 628 & 632 & 641 & 589 \\
\hline M10 & 712 & 689 & 694 & 732 & 785 & 715 \\
\hline
\end{tabular}


Table 27: Insulin ( $\mu \mathrm{U} / \mathrm{mL})$ Start to $17 \mathrm{~km}$

\begin{tabular}{|c|c|c|c|c|c|c|c|c|}
\hline \multirow{2}{*}{ Participant } & \multicolumn{2}{|c|}{ Pre Drink Pre Race } & \multicolumn{2}{|c|}{ Post Drink Pre Race } & \multicolumn{2}{|c|}{$6 \mathrm{~km}$} & \multicolumn{2}{|c|}{17 km } \\
\hline & ED & PLA & ED & PLA & ED & PLA & ED & PLA \\
\hline F1 & 63 & 31 & 120 & 61 & 22 & 36 & 24 & 51 \\
\hline F3 & 26 & 39 & 53 & 38 & 33 & 23 & 31 & 27 \\
\hline F4 & 21 & 35 & 49 & 25 & 20 & 22 & 16 & 31 \\
\hline F5 & 17 & 14 & 39 & 16 & 16 & 15 & 14 & 22 \\
\hline F6 & 23 & 29 & 48 & 27 & 20 & 19 & 17 & 23 \\
\hline F8 & 21 & 24 & 78 & 14 & 37 & 44 & 24 & 21 \\
\hline F9 & 15 & 11 & 38 & 14 & 12 & 13 & 17 & 13 \\
\hline M1 & 21 & 23 & 58 & 20 & 46 & 16 & 26 & 19 \\
\hline M2 & 28 & 21 & 55 & 25 & 25 & 16 & 19 & 14 \\
\hline M3 & 44 & 35 & 85 & 33 & 28 & 33 & 24 & 32 \\
\hline M4 & 17 & 22 & 82 & 27 & 15 & 13 & 16 & 14 \\
\hline M7 & 31 & 25 & 80 & 27 & 42 & 22 & 25 & 24 \\
\hline M8 & 24 & 27 & 41 & 26 & 22 & 21 & 16 & 21 \\
\hline M9 & 36 & 28 & 67 & 29 & 23 & 26 & 22 & 19 \\
\hline M10 & 15 & 21 & 47 & 16 & 21 & 13 & 16 & 13 \\
\hline
\end{tabular}


Table 28: Insulin $(\mu \mathrm{U} / \mathrm{mL}) 23 \mathrm{~km}$ to $34 \mathrm{~km}$

\begin{tabular}{|c|c|c|c|c|c|c|}
\hline \multirow{2}{*}{ Participant } & \multicolumn{2}{|c|}{$\mathbf{2 3} \mathbf{~ k m}$} & \multicolumn{2}{c|}{$\mathbf{2 9} \mathbf{~ k m}$} & \multicolumn{2}{c|}{ 34 km } \\
& ED & PLA & ED & PLA & ED & PLA \\
\hline F1 & 33 & 31 & 20 & 27 & 48 & 49 \\
\hline F3 & 28 & 29 & 21 & 27 & 26 & 28 \\
\hline F4 & 25 & 26 & 19 & 18 & 13 & 18 \\
\hline F5 & 13 & 16 & 15 & 12 & 14 & 11 \\
\hline F6 & 22 & 16 & 15 & 12 & 15 & $16^{\wedge}$ \\
\hline F8 & 16 & 16 & 24 & 18 & 19 & 14 \\
\hline F9 & 11 & 13 & 11 & 8 & 6 & 10 \\
\hline M1 & 22 & 22 & 19 & 15 & 17 & 15 \\
\hline M2 & 24 & 16 & 20 & 11 & 21 & 21 \\
\hline M3 & 23 & 26 & 21 & 28 & 19 & 24 \\
\hline M4 & 11 & 18 & 16 & 11 & 11 & 14 \\
\hline M7 & 24 & 28 & 27 & 22 & 18 & 20 \\
\hline M8 & 20 & 18 & 17 & 26 & 17 & 19 \\
\hline M9 & 17 & 19 & 21 & 27 & 28 & 18 \\
\hline M10 & 16 & 15 & 10 & 15 & 17 & 9 \\
\hline \multicolumn{7}{|l}{$\wedge$ Blood draw missed but data point estimated using method described in Appendix F. } \\
\hline
\end{tabular}


Table 29: Glucose (mg/dL) Start to $17 \mathrm{~km}$

\begin{tabular}{|c|c|c|c|c|c|c|c|c|}
\hline \multirow{2}{*}{ Participant } & \multicolumn{2}{|c|}{ Pre Drink Pre Race } & \multicolumn{2}{|c|}{ Post Drink Pre Race } & \multicolumn{2}{|c|}{$6 \mathrm{~km}$} & \multicolumn{2}{|c|}{17 km } \\
\hline & ED & PLA & ED & PLA & ED & PLA & ED & PLA \\
\hline F1 & 77 & 87 & 68 & 78 & 62 & 91 & 77 & 98 \\
\hline F3 & 85 & 78 & 66 & 77 & 63 & 79 & 81 & 84 \\
\hline F4 & 77 & 85 & 72 & 74 & 63 & 78 & 88 & 86 \\
\hline F5 & 82 & 83 & 46 & 77 & 54 & 80 & 80 & 87 \\
\hline F6 & 80 & 85 & 66 & 79 & 69 & 75 & 88 & 79 \\
\hline F8 & 83 & 70 & 97 & 64 & 87 & 78 & 80 & 90 \\
\hline F9 & 80 & 84 & 95 & 83 & 62 & 84 & 65 & 82 \\
\hline M1 & 93 & 86 & 123 & 82 & 81 & 84 & 67 & 84 \\
\hline M2 & 91 & 87 & 95 & 85 & 83 & 87 & 86 & 94 \\
\hline M3 & 91 & 92 & 60 & 82 & 67 & 82 & 85 & 85 \\
\hline M4 & 88 & 83 & 95 & 79 & 42 & 74 & 63 & 83 \\
\hline M7 & 80 & 80 & 95 & 77 & 61 & 92 & 86 & 95 \\
\hline M8 & 82 & 78 & 88 & 65 & 52 & 62 & 69 & 75 \\
\hline M9 & 82 & 89 & 58 & 79 & 40 & 76 & 69 & 82 \\
\hline M10 & 74 & 77 & 108 & 73 & 60 & 70 & 58 & 75 \\
\hline
\end{tabular}


Table 40: Glucose (mg/dL) $23 \mathrm{~km}$ to $34 \mathrm{~km}$

\begin{tabular}{|c|c|c|c|c|c|c|}
\hline \multirow{2}{*}{ Participant } & \multicolumn{2}{|c|}{$\mathbf{2 3} \mathbf{~ k m}$} & \multicolumn{2}{c|}{$\mathbf{2 9} \mathbf{~ k m}$} & \multicolumn{2}{c|}{ 34 km } \\
& ED & PLA & ED & PLA & ED & PLA \\
\hline F1 & 80 & 93 & 76 & 86 & 65 & 84 \\
\hline F3 & 79 & 76 & 83 & 74 & 77 & 76 \\
\hline F4 & 90 & 89 & 91 & 77 & 88 & 69 \\
\hline F5 & 70 & 80 & 69 & 81 & 60 & 77 \\
\hline F6 & 84 & 79 & 85 & 64 & 83 & $75^{\wedge}$ \\
\hline F8 & 86 & 76 & 71 & 68 & 75 & 79 \\
\hline F9 & 69 & 82 & 68 & 80 & 65 & 79 \\
\hline M1 & 69 & 87 & 72 & 73 & 73 & 70 \\
\hline M2 & 81 & 79 & 71 & 71 & 67 & 64 \\
\hline M3 & 87 & 88 & 86 & 89 & 86 & 84 \\
\hline M4 & 66 & 82 & 63 & 82 & 69 & 84 \\
\hline M7 & 83 & 93 & 72 & 77 & 64 & 73 \\
\hline M8 & 70 & 74 & 70 & 71 & 60 & 67 \\
\hline M9 & 64 & 81 & 69 & 82 & 65 & 75 \\
\hline M10 & 65 & 67 & 64 & 58 & 54 & 56 \\
\hline \multicolumn{2}{|l|}{ ^Blood draw missed but data point estimated using method described in Appendix F. } \\
\hline
\end{tabular}


Table 30: Free Fatty Acids (mmol/L) Start to $17 \mathrm{~km}$

\begin{tabular}{|c|c|c|c|c|c|c|c|c|}
\hline \multirow{2}{*}{ Participant } & \multicolumn{2}{|c|}{$\begin{array}{c}\text { Pre Drink Pre } \\
\text { Race }\end{array}$} & \multicolumn{2}{c|}{$\begin{array}{c}\text { Post Drink Pre } \\
\text { Race }\end{array}$} & \multicolumn{2}{c|}{ 6 km } & \multicolumn{2}{c|}{ 17 km } \\
& ED & PLA & ED & PLA & ED & PLA & ED & PLA \\
\hline F1 & 0.42 & 0.32 & 0.21 & 0.33 & 0.18 & 0.20 & 0.32 & 0.33 \\
\hline F3 & 0.43 & 0.28 & 0.13 & 0.32 & 0.09 & 0.43 & 0.25 & 0.53 \\
\hline F4 & 0.31 & 0.27 & 0.11 & 0.26 & 0.09 & 0.16 & 0.10 & 0.30 \\
\hline F5 & 0.28 & 0.18 & 0.17 & 0.20 & 0.15 & 0.18 & 0.15 & 0.23 \\
\hline F6 & 0.13 & 0.26 & 0.09 & 0.18 & 0.05 & 0.16 & 0.07 & 0.19 \\
\hline F8 & 0.31 & 0.16 & 0.15 & 0.10 & 0.13 & 0.18 & 0.28 & 0.39 \\
\hline F9 & 0.25 & 0.34 & 0.09 & 0.19 & 0.08 & 0.17 & 0.11 & 0.20 \\
\hline M1 & 0.52 & 0.32 & 0.15 & 0.23 & 0.13 & 0.25 & 0.11 & 0.40 \\
\hline M2 & 0.36 & 0.28 & 0.12 & 0.26 & 0.08 & 0.13 & 0.06 & 0.11 \\
\hline M3 & 0.20 & 0.15 & 0.12 & 0.18 & 0.19 & 0.19 & 0.14 & 0.23 \\
\hline M4 & 0.24 & 0.24 & 0.05 & 0.16 & 0.02 & 0.13 & 0.08 & 0.14 \\
\hline M7 & 0.34 & 0.19 & 0.16 & 0.16 & 0.10 & 0.16 & 0.10 & 0.20 \\
\hline M8 & 0.29 & 0.40 & 0.22 & 0.34 & 0.14 & 0.19 & 0.16 & 0.23 \\
\hline M9 & 0.05 & 0.12 & 0.05 & 0.12 & 0.08 & 0.19 & 0.07 & 0.13 \\
\hline M10 & 0.14 & 0.25 & 0.07 & 0.16 & 0.03 & 0.14 & 0.07 & 0.25 \\
\hline
\end{tabular}


Table 31: Free Fatty Acids (mmol/L) $23 \mathrm{~km}$ to $34 \mathrm{~km}$

\begin{tabular}{|c|c|c|c|c|c|c|}
\hline \multirow{2}{*}{ Participant } & \multicolumn{2}{|c|}{$23 \mathbf{~ k m}$} & \multicolumn{2}{c|}{ 29 km } & \multicolumn{2}{c|}{ 34 km } \\
& ED & PLA & ED & PLA & ED & PLA \\
\hline F1 & 0.44 & 0.30 & 0.67 & 0.29 & 0.66 & 0.30 \\
\hline F3 & 0.29 & 0.53 & 0.42 & 0.50 & 0.54 & 0.63 \\
\hline F4 & 0.12 & 0.29 & 0.13 & 0.24 & 0.12 & 0.26 \\
\hline F5 & 0.14 & 0.21 & 0.16 & 0.29 & 0.20 & 0.35 \\
\hline F6 & 0.09 & 0.20 & 0.09 & 0.17 & 0.08 & $0.22 \wedge$ \\
\hline F8 & 0.34 & 0.38 & 0.41 & 0.64 & 0.57 & 0.54 \\
\hline F9 & 0.13 & 0.21 & 0.23 & 0.21 & 0.14 & 0.24 \\
\hline M1 & 0.21 & 0.35 & 0.16 & 0.32 & 0.27 & 0.38 \\
\hline M2 & 0.14 & 0.10 & 0.13 & 0.12 & 0.12 & 0.10 \\
\hline M3 & 0.15 & 0.29 & 0.18 & 0.20 & 0.21 & 0.19 \\
\hline M4 & 0.10 & 0.22 & 0.13 & 0.15 & 0.12 & 0.18 \\
\hline M7 & 0.14 & 0.20 & 0.12 & 0.24 & 0.16 & 0.24 \\
\hline M8 & 0.19 & 0.27 & 0.20 & 0.19 & 0.24 & 0.22 \\
\hline M9 & 0.05 & 0.13 & 0.11 & 0.21 & 0.13 & 0.14 \\
\hline M10 & 0.07 & 0.33 & 0.10 & 0.37 & 0.10 & 0.47 \\
\hline ^Blood draw missed but data point estimated using method described in \\
\hline \multicolumn{7}{|l}{ Appendix F. } \\
\hline
\end{tabular}


Table 32: Glycerol (mmol/L) Start to $17 \mathrm{~km}$

\begin{tabular}{|c|c|c|c|c|c|c|c|c|}
\hline \multirow{2}{*}{ Participant } & \multicolumn{2}{|c|}{$\begin{array}{c}\text { Pre Drink Pre } \\
\text { Race }\end{array}$} & \multicolumn{2}{c|}{$\begin{array}{c}\text { Post Drink Pre } \\
\text { Race }\end{array}$} & \multicolumn{2}{c|}{ 6 km } & \multicolumn{2}{c|}{17 km } \\
& ED & PLA & ED & PLA & ED & PLA & ED & PLA \\
\hline F1 & 0.06 & 0.05 & 0.06 & 0.07 & 0.13 & 0.20 & 0.39 & 0.21 \\
\hline F3 & 0.01 & 0.03 & 0.04 & 0.07 & 0.13 & 0.32 & 0.32 & 0.51 \\
\hline F4 & 0.10 & 0.07 & 0.06 & 0.08 & 0.26 & 0.20 & 0.47 & 0.38 \\
\hline F5 & 0.05 & 0.04 & 0.04 & 0.07 & 0.10 & 0.17 & 0.22 & 0.31 \\
\hline F6 & 0.02 & 0.04 & 0.02 & 0.03 & 0.06 & 0.09 & 0.11 & 0.17 \\
\hline F8 & 0.05 & 0.03 & 0.05 & 0.02 & 0.15 & 0.12 & 0.28 & 0.28 \\
\hline F9 & 0.08 & 0.09 & 0.05 & 0.09 & 0.23 & 0.31 & 0.45 & 0.57 \\
\hline M1 & 0.07 & 0.04 & 0.04 & 0.03 & 0.13 & 0.12 & 0.21 & 0.25 \\
\hline M2 & 0.08 & 0.06 & 0.04 & 0.09 & 0.14 & 0.12 & 0.27 & 0.20 \\
\hline M3 & 0.04 & 0.02 & 0.04 & 0.03 & 0.09 & 0.08 & 0.17 & 0.12 \\
\hline M4 & 0.04 & 0.02 & 0.04 & 0.04 & 0.10 & 0.10 & 0.18 & 0.18 \\
\hline M7 & 0.05 & 0.03 & 0.02 & 0.03 & 0.09 & 0.09 & 0.18 & 0.16 \\
\hline M8 & 0.05 & 0.11 & 0.07 & 0.13 & 0.20 & 0.27 & 0.30 & 0.43 \\
\hline M9 & 0.01 & 0.02 & 0.03 & 0.02 & 0.06 & 0.03 & 0.11 & 0.06 \\
\hline M10 & 0.04 & 0.04 & 0.04 & 0.04 & 0.08 & 0.11 & 0.14 & 0.23 \\
\hline
\end{tabular}


Table 33: Glycerol (mmol/L) $23 \mathrm{~km}$ to $34 \mathrm{~km}$

\begin{tabular}{|c|c|c|c|c|c|c|}
\hline \multirow{2}{*}{ Participant } & \multicolumn{2}{|c|}{$23 \mathbf{~ k m}$} & \multicolumn{2}{c|}{ 29 km } & \multicolumn{2}{c|}{34 km } \\
& ED & PLA & ED & PLA & ED & PLA \\
\hline F1 & 0.46 & 0.21 & 0.54 & 0.23 & 0.55 & 0.28 \\
\hline F3 & 0.38 & 0.53 & 0.46 & 0.59 & 0.48 & 0.61 \\
\hline F4 & 0.56 & 0.44 & 0.66 & 0.46 & 0.70 & 0.50 \\
\hline F5 & 0.29 & 0.32 & 0.33 & 0.37 & 0.35 & 0.40 \\
\hline F6 & 0.14 & 0.21 & 0.16 & 0.22 & 0.18 & $0.27 \wedge$ \\
\hline F8 & 0.37 & 0.31 & 0.43 & 0.36 & 0.49 & 0.41 \\
\hline F9 & 0.57 & 0.65 & 0.61 & 0.72 & 0.68 & 0.74 \\
\hline M1 & 0.23 & 0.28 & 0.29 & 0.28 & 0.32 & 0.28 \\
\hline M2 & 0.32 & 0.25 & 0.33 & 0.26 & 0.38 & 0.30 \\
\hline M3 & 0.18 & 0.12 & 0.20 & 0.14 & 0.21 & 0.14 \\
\hline M4 & 0.21 & 0.21 & 0.25 & 0.24 & 0.28 & 0.26 \\
\hline M7 & 0.23 & 0.19 & 0.26 & 0.21 & 0.27 & 0.22 \\
\hline M8 & 0.37 & 0.44 & 0.38 & 0.46 & 0.41 & 0.54 \\
\hline M9 & 0.11 & 0.07 & 0.14 & 0.07 & 0.13 & 0.08 \\
\hline M10 & 0.19 & 0.29 & 0.20 & 0.35 & 0.23 & 0.40 \\
\hline ^Blood draw missed but data point estimated using method \\
described in Appendix F. \\
\hline \multicolumn{7}{|l|}{}
\end{tabular}


Table 34: Lactate (mmol/L)

\begin{tabular}{|c|c|c|c|c|c|c|c|c|}
\hline \multirow{2}{*}{ Participant } & \multicolumn{2}{|c|}{$\begin{array}{c}\text { Pre Drink Pre } \\
\text { Race }\end{array}$} & \multicolumn{2}{c|}{$\begin{array}{c}\text { Post Drink Pre } \\
\text { Race }\end{array}$} & \multicolumn{2}{c|}{ 6 km } & \multicolumn{2}{c|}{ 17 km } \\
& ED & PLA & ED & PLA & ED & PLA & ED & PLA \\
\hline F1 & 0.7 & 0.9 & 1.7 & 0.9 & 2.8 & 3.2 & 2.6 & 2.5 \\
\hline F3 & 0.7 & 0.7 & 1.4 & 0.6 & 2.6 & 0.8 & 1.7 & 0.8 \\
\hline F4 & 0.6 & 0.7 & 1.9 & 0.7 & 4.8 & 3.4 & 6.0 & 3.1 \\
\hline F5 & 0.7 & 0.7 & 1.7 & 0.8 & 4.6 & 2.6 & 4.4 & 2.5 \\
\hline F6 & 0.8 & 0.6 & 2.0 & 0.8 & 3.9 & 1.5 & 5.3 & 1.8 \\
\hline F8 & 0.8 & 0.8 & 1.6 & 0.6 & 5.9 & 3.5 & 4.6 & 2.7 \\
\hline F9 & 0.7 & 0.6 & 1.7 & 0.6 & 6.4 & 2.5 & 5.2 & 1.5 \\
\hline M1 & 0.7 & 0.6 & 2.2 & 0.7 & 4.3 & 2.7 & 3.5 & 1.2 \\
\hline M2 & 0.6 & 0.7 & 1.4 & 0.7 & 8.0 & 6.9 & 9.3 & 8.7 \\
\hline M3 & 0.9 & 0.8 & 1.7 & 0.8 & 3.5 & 2.9 & 4.9 & 2.6 \\
\hline M4 & 0.8 & 0.7 & 2.0 & 0.8 & 4.3 & 1.9 & 3.4 & 3.2 \\
\hline M7 & 0.5 & 0.6 & 1.2 & 0.5 & 6.7 & 7.8 & 7.5 & 5.4 \\
\hline M8 & 1.3 & 0.8 & 1.7 & 0.9 & 4.5 & 4.8 & 3.6 & 4.2 \\
\hline M9 & 0.8 & 0.8 & 1.7 & 0.7 & 5.1 & 3.0 & 4.6 & 2.5 \\
\hline M10 & 0.5 & 0.6 & 1.1 & 0.7 & 4.3 & 4.8 & 5.5 & 3.8 \\
\hline
\end{tabular}


Table 35: Lactate (mmol/L) $23 \mathrm{~km}$ to $34 \mathrm{~km}$

\begin{tabular}{|c|c|c|c|c|c|c|}
\hline \multirow{2}{*}{ Participant } & \multicolumn{2}{|c|}{$23 \mathbf{~ k m}$} & \multicolumn{2}{c|}{ 29 km } & \multicolumn{2}{c|}{ 34 km } \\
& ED & PLA & ED & PLA & ED & PLA \\
\hline F1 & 2.7 & 2.2 & 2.2 & 2.0 & 2.5 & 2.8 \\
\hline F3 & 1.4 & 0.7 & 1.5 & 0.8 & 1.2 & 0.9 \\
\hline F4 & 5.4 & 3.8 & 5.0 & 2.8 & 6.3 & 2.9 \\
\hline F5 & 4.1 & 2.2 & 3.5 & 2.4 & 3.4 & 1.9 \\
\hline F6 & 4.6 & 1.5 & 4.3 & 2.3 & 4.9 & $2.37^{\wedge}$ \\
\hline F8 & 4.2 & 2.2 & 4.2 & 1.4 & 3.7 & 1.8 \\
\hline F9 & 5.0 & 1.5 & 4.1 & 1.3 & 4.5 & 1.5 \\
\hline M1 & 2.6 & 2.3 & 3.5 & 1.4 & 3.7 & 1.1 \\
\hline M2 & 8.6 & 7.2 & 7.0 & 6.1 & 6.9 & 7.0 \\
\hline M3 & 5.4 & 2.0 & 4.5 & 4.5 & 5.4 & 6.0 \\
\hline M4 & 2.5 & 1.9 & 4.7 & 2.6 & 4.2 & 3.1 \\
\hline M7 & 6.5 & 3.4 & 5.0 & 1.9 & 4.7 & 1.9 \\
\hline M8 & 2.8 & 2.9 & 2.6 & 2.7 & 2.4 & 2.3 \\
\hline M9 & 3.5 & 2.1 & 4.2 & 1.7 & 3.4 & 1.5 \\
\hline M10 & 5.1 & 2.3 & 4.6 & 1.8 & 4.6 & 1.4 \\
\hline ^Blood draw missed but data point estimated using method described in \\
\hline \multicolumn{7}{|l}{ Appendix F. }
\end{tabular}


Table 36: Myoglobin (ng/mL)

\begin{tabular}{|c|c|c|c|c|c|c|c|c|c|c|c|c|}
\hline \multirow{2}{*}{ Participant } & \multicolumn{2}{|c|}{$\begin{array}{c}\text { Pre Drink Pre } \\
\text { Race }\end{array}$} & \multicolumn{2}{|c|}{$\begin{array}{c}\text { Post Drink Pre } \\
\text { Race }\end{array}$} & \multicolumn{2}{|c|}{$6 \mathrm{~km}$} & \multicolumn{2}{|c|}{$23 \mathrm{~km}$} & \multicolumn{2}{|c|}{29 km } & \multicolumn{2}{|c|}{34 km } \\
\hline & ED & PLA & ED & PLA & ED & PLA & ED & PLA & ED & PLA & ED & PLA \\
\hline F1 & 11 & 12 & 9 & 9 & 9 & 6 & 13 & 6 & 9 & 13 & 12 & 13 \\
\hline F3 & 16 & 7 & 16 & 8 & 12 & 8 & 12 & 7 & 15 & 7 & 14 & 6 \\
\hline F4 & 15 & 18 & 12 & 15 & 9 & 17 & 10 & 17 & 16 & 16 & 24 & 18 \\
\hline F5 & 15 & 12 & 15 & 10 & 9 & 8 & 6 & 12 & 10 & 11 & 9 & 10 \\
\hline F6 & 20 & 14 & 31 & 18 & 16 & 16 & 21 & 19 & 17 & 19 & 21 & $21^{\wedge}$ \\
\hline F8 & 14 & 23 & 14 & 18 & 12 & 13 & 17 & 15 & 22 & 11 & 19 & 13 \\
\hline F9 & 19 & 20 & 23 & 14 & 18 & 17 & 17 & 15 & 15 & 18 & 22 & 22 \\
\hline M1 & 11 & 16 & 14 & 16 & 10 & 18 & 11 & 16 & 13 & 17 & 13 & 16 \\
\hline M2 & 17 & 21 & 15 & 19 & 13 & 20 & 21 & 26 & 21 & 27 & 20 & 31 \\
\hline M3 & 5 & 7 & 4 & 9 & 5 & 7 & 5 & 7 & 5 & 4 & 7 & 6 \\
\hline M4 & 17 & 14 & 16 & 14 & 18 & 12 & 16 & 13 & 14 & 18 & 13 & 24 \\
\hline M7 & 22 & 25 & 37 & 29 & 39 & 26 & 39 & 34 & 35 & 38 & 54 & 34 \\
\hline M8 & 15 & 11 & 11 & 11 & 7 & 12 & 12 & 13 & 13 & 14 & 17 & 17 \\
\hline M9 & 46 & 65 & 54 & 70 & 52 & 66 & 74 & 77 & 57 & 84 & 68 & 68 \\
\hline M10 & 34 & 16 & 20 & 12 & 20 & 16 & 18 & 21 & 30 & 13 & 21 & 12 \\
\hline
\end{tabular}


Table 37: Caffeine (ng/mL)

\begin{tabular}{|c|c|c|c|c|c|c|}
\hline \multirow{2}{*}{ Participant } & \multicolumn{2}{|c|}{$\begin{array}{c}\text { Pre Drink Pre } \\
\text { Race }\end{array}$} & \multicolumn{2}{c|}{ Post Drink Pre } & \multicolumn{2}{c|}{ R4 km } \\
& ED & PLA & ED & PLA & ED & PLA \\
\hline F1 & 102 & 108 & 2396 & 127 & 2899 & 88 \\
\hline F3 & 2643 & 1439 & 3410 & 1485 & 2205 & 1428 \\
\hline F4 & 1097 & 1302 & 2831 & 1283 & 3697 & 1790 \\
\hline F5 & 1698 & 1089 & 2474 & 1890 & 2865 & 1669 \\
\hline F6 & 469 & 820 & 2297 & 451 & 2703 & $482^{\wedge}$ \\
\hline F8 & 45 & 22 & 1450 & 28 & 1405 & 18 \\
\hline F9 & 80 & 110 & 1363 & 98 & 3238 & 99 \\
\hline M1 & 43 & 111 & 2186 & 98 & 1962 & 86 \\
\hline M2 & 190 & 115 & 509 & 126 & 712 & 89 \\
\hline M3 & 108 & 44 & 2525 & 44 & 2923 & 43 \\
\hline M4 & 1611 & 168 & 3015 & 226 & 2713 & 173 \\
\hline M7 & 1567 & 1422 & 3236 & 1330 & 3898 & 872 \\
\hline M8 & 321 & 146 & 2598 & 161 & 2249 & 111 \\
\hline M9 & 2205 & 2176 & 3484 & 1593 & 3316 & 1330 \\
\hline M10 & 820 & 135 & 2473 & 119 & 2439 & 80 \\
\hline Blood draw missed but data point estimated using method described in \\
\hline \multicolumn{7}{|l|}{ Appendix F. } \\
\hline
\end{tabular}


Table 38: Epinephrine (pg/mL)

\begin{tabular}{|c|c|c|c|c|c|c|c|c|c|c|}
\hline \multirow{2}{*}{ Participant } & \multicolumn{2}{|c|}{$\begin{array}{l}\text { Pre Drink Pre } \\
\text { Race }\end{array}$} & \multicolumn{2}{|c|}{$\begin{array}{l}\text { Post Drink Pre } \\
\text { Race }\end{array}$} & \multicolumn{2}{|c|}{17 km } & \multicolumn{2}{|c|}{29 km } & \multicolumn{2}{|c|}{34 km } \\
\hline & ED & PLA & ED & PLA & ED & PLA & ED & PLA & ED & PLA \\
\hline F1 & 149 & 128 & 139 & 92 & 349 & 305 & 260 & 286 & 559 & 417 \\
\hline F3 & 123 & 78 & 117 & 108 & 374 & 146 & 367 & 174 & 330 & 206 \\
\hline F4 & 82 & 128 & 148 & 86 & 613 & 322 & 609 & 355 & 605 & 374 \\
\hline F5 & 130 & 126 & 190 & 90 & 417 & 282 & 426 & 316 & 422 & 302 \\
\hline F6 & 0 & 32 & 12 & 0 & 128 & 235 & 243 & 211 & 319 & $218^{\wedge}$ \\
\hline F8 & 29 & 18 & 58 & 5 & 542 & 183 & 496 & 182 & 682 & 247 \\
\hline F9 & 14 & 0 & 2 & 30 & 458 & 115 & 557 & 112 & 935 & 121 \\
\hline M1 & 96 & 74 & 68 & 63 & 439 & 207 & 489 & 204 & 589 & 273 \\
\hline M2 & 71 & 78 & 48 & 72 & 597 & 322 & 892 & 430 & 1464 & 1211 \\
\hline M3 & 102 & 102 & 71 & 52 & 435 & 225 & 384 & 277 & 454 & 294 \\
\hline M4 & 79 & 53 & 52 & 54 & 589 & 199 & 769 & 214 & 481 & 144 \\
\hline M7 & 0 & 49 & 0 & 34 & 579 & 302 & 548 & 293 & 723 & 323 \\
\hline M8 & 13 & 0 & 0 & 0 & 510 & 331 & 454 & 271 & 581 & 375 \\
\hline M9 & 30 & 0 & 0 & 0 & 444 & 171 & 483 & 216 & 518 & 238 \\
\hline M10 & 226 & 0 & 0 & 0 & 650 & 285 & 658 & 377 & 764 & 489 \\
\hline
\end{tabular}


Table 50: Norepinephrine (pg/mL)

\begin{tabular}{|c|c|c|c|c|c|c|c|c|c|c|}
\hline \multirow{2}{*}{ Participant } & \multicolumn{2}{|c|}{$\begin{array}{l}\text { Pre Drink Pre } \\
\text { Race }\end{array}$} & \multicolumn{2}{|c|}{$\begin{array}{c}\text { Post Drink Pre } \\
\text { Race }\end{array}$} & \multicolumn{2}{|c|}{17 km } & \multicolumn{2}{|c|}{29 km } & \multicolumn{2}{|c|}{$34 \mathrm{~km}$} \\
\hline & ED & PLA & ED & PLA & ED & PLA & ED & PLA & ED & PLA \\
\hline F1 & 300 & 302 & 1196 & 565 & 3090 & 2829 & 1861 & 2780 & 1815 & 1947 \\
\hline F3 & 371 & 675 & 508 & 1040 & 1136 & 2079 & 1481 & 1344 & 1391 & 1581 \\
\hline F4 & 136 & 96 & 713 & 489 & 4264 & 2426 & 2203 & 2157 & 2487 & 2900 \\
\hline F5 & 305 & 224 & 199 & 988 & 554 & 1064 & 787 & 1301 & 1216 & 721 \\
\hline F6 & 143 & 143 & 398 & 307 & 4150 & 1625 & 3986 & 2144 & 2408 & $2443^{\wedge}$ \\
\hline F8 & 340 & 143 & 347 & 261 & 3785 & 2664 & 3617 & 2103 & 4555 & 2812 \\
\hline F9 & 407 & 182 & 520 & 570 & 2396 & 1446 & 3546 & 1518 & 4268 & 1686 \\
\hline M1 & 479 & 557 & 803 & 1235 & 3434 & 2908 & 4187 & 2973 & 3992 & 3391 \\
\hline M2 & 625 & 729 & 1379 & 1418 & 4796 & 4660 & 4307 & 4954 & 5040 & 4937 \\
\hline M3 & 398 & 430 & 538 & 693 & 2362 & 1527 & 2151 & 2184 & 2307 & 2537 \\
\hline M4 & 411 & 432 & 849 & 511 & 2254 & 1631 & 3627 & 1779 & 2647 & 2402 \\
\hline M7 & 435 & 631 & 513 & 776 & 3612 & 3214 & 3687 & 2829 & 3543 & 2983 \\
\hline M8 & 580 & 602 & 1055 & 1676 & 2770 & 2692 & 2289 & 2670 & 2530 & 3421 \\
\hline M9 & 438 & 363 & 558 & 525 & 2541 & 1637 & 2401 & 1595 & 2266 & 1238 \\
\hline M10 & 544 & 175 & 707 & 394 & 1597 & 1186 & 1357 & 1102 & 1371 & 1297 \\
\hline
\end{tabular}


Table 39: $\beta$-Endorphins (ng/mL)

\begin{tabular}{|c|c|c|c|c|c|c|}
\hline \multirow{2}{*}{ Participant } & \multicolumn{2}{|c|}{ Pre Drink Pre } & \multicolumn{2}{c|}{ Post Drink Pre } & \multicolumn{2}{c|}{ 34 km } \\
& ED & PLA & ED & PLA & ED & PLA \\
\hline F1 & 0.06 & 0.08 & 0.04 & 0.05 & .04 & .06 \\
\hline F3 & 0.04 & 0.03 & 0.02 & 0.03 & 0.06 & 0.08 \\
\hline F4 & 0.04 & 0.03 & 0.03 & 0.04 & 0.05 & 0.05 \\
\hline F5 & 0.03 & 0.02 & 0.02 & 0.03 & 0.04 & 0.04 \\
\hline F6 & 0.03 & 0.05 & 0.03 & 0.04 & $.06 \wedge$ & .05 \\
\hline F8 & 0.04 & 0.04 & 0.03 & 0.03 & 0.08 & 0.07 \\
\hline F9 & 0.03 & 0.05 & 0.04 & 0.04 & 0.05 & 0.05 \\
\hline M1 & 0.03 & 0.03 & 0.05 & 0.35 & 0.05 & 0.05 \\
\hline M2 & 0.08 & 0.05 & 0.04 & 0.05 & 0.07 & 0.08 \\
\hline M3 & 0.04 & 0.06 & 0.05 & 0.04 & 0.06 & 0.06 \\
\hline M4 & 0.01 & 0.03 & 0.03 & 0.03 & 0.04 & 0.03 \\
\hline M7 & 0.05 & 0.05 & 0.05 & 0.07 & 0.15 & 0.08 \\
\hline M8 & 0.04 & 0.05 & 0.02 & 0.04 & 0.07 & 0.07 \\
\hline M9 & 0.04 & 0.02 & 0.03 & 0.00 & 0.04 & 0.03 \\
\hline M10 & 0.05 & 0.06 & 0.03 & 0.06 & 0.07 & 0.16 \\
\hline ^Blood draw missed but data point estimated using method described in \\
\hline \multicolumn{7}{|l|}{ Appendix F. } \\
\hline
\end{tabular}


Table 40: Cortisol ( $\mu \mathrm{g} / \mathrm{dL})$ Start to $17 \mathrm{~km}$

\begin{tabular}{|c|c|c|c|c|c|c|c|c|}
\hline \multirow{2}{*}{ Participant } & \multicolumn{2}{|c|}{$\begin{array}{c}\text { Pre Drink Pre } \\
\text { Race }\end{array}$} & \multicolumn{2}{c|}{$\begin{array}{c}\text { Post Drink Pre } \\
\text { Race }\end{array}$} & \multicolumn{2}{c|}{ 6 km } & \multicolumn{2}{c|}{17 km } \\
& ED & PLA & ED & PLA & ED & PLA & ED & PLA \\
\hline F1 & 31 & 21 & 24 & 28 & 22 & 23 & 18 & 24 \\
\hline F3 & 20 & 15 & 20 & 13 & 17 & 19 & 26 & 24 \\
\hline F4 & 26 & 20 & 27 & 19 & 23 & 24 & 33 & 29 \\
\hline F5 & 24 & 25 & 25 & 22 & 25 & 29 & 29 & 28 \\
\hline F6 & 43 & 51 & 38 & 49 & 39 & 37 & 45 & 46 \\
\hline F8 & 21 & 25 & 22 & 19 & 24 & 22 & 34 & 33 \\
\hline F9 & 31 & 41 & 34 & 35 & 27 & 31 & 26 & 28 \\
\hline M1 & 45 & 25 & 39 & 27 & 37 & 20 & 30 & 20 \\
\hline M2 & 18 & 25 & 27 & 26 & 34 & 29 & 34 & 32 \\
\hline M3 & 19 & 18 & 20 & 15 & 22 & 15 & 26 & 20 \\
\hline M4 & 44 & 52 & 42 & 47 & 53 & 44 & 69 & 46 \\
\hline M7 & 24 & 21 & 28 & 16 & 23 & 16 & 29 & 25 \\
\hline M8 & 27 & 31 & 23 & 25 & 25 & 21 & 29 & 29 \\
\hline M9 & 24 & 21 & 17 & 16 & 17 & 13 & 27 & 18 \\
\hline M10 & 22 & 23 & 26 & 27 & 30 & 29 & 19 & 36 \\
\hline
\end{tabular}


Table 41: Cortisol ( $\mu \mathrm{g} / \mathrm{dL}) 23 \mathrm{~km}$ to $34 \mathrm{~km}$

\begin{tabular}{|c|c|c|c|c|c|c|}
\hline \multirow{2}{*}{ Participant } & \multicolumn{2}{|c|}{$23 \mathbf{k m}$} & \multicolumn{2}{c|}{ 29 km } & \multicolumn{2}{c|}{ 34 km } \\
& ED & PLA & ED & PLA & ED & PLA \\
\hline F1 & 15 & 32 & 15 & 35 & 14 & 35 \\
\hline F3 & 31 & 21 & 31 & 18 & 31 & 20 \\
\hline F4 & 34 & 31 & 41 & 38 & 37 & 34 \\
\hline F5 & 32 & 29 & 37 & 24 & 36 & 28 \\
\hline F6 & 44 & 44 & 46 & 39 & 50 & $45^{\wedge}$ \\
\hline F8 & 38 & 44 & 38 & 31 & 41 & 30 \\
\hline F9 & 27 & 26 & 32 & 27 & 31 & 27 \\
\hline M1 & 29 & 20 & 27 & 21 & 25 & 21 \\
\hline M2 & 36 & 36 & 38 & 33 & 39 & 34 \\
\hline M3 & 31 & 20 & 28 & 20 & 26 & 20 \\
\hline M4 & 70 & 57 & 68 & 55 & 57 & 54 \\
\hline M7 & 33 & 28 & 30 & 29 & 36 & 29 \\
\hline M8 & 34 & 36 & 35 & 36 & 35 & 37 \\
\hline M9 & 30 & 18 & 32 & 19 & 32 & 20 \\
\hline M10 & 26 & 37 & 32 & 40 & 36 & 35 \\
\hline ^Blood draw missed but data point estimated using method described in \\
\hline
\end{tabular}


APPENDIX C: STUDY SUPPLEMENT DETAIL

\begin{tabular}{|c|c|c|}
\hline & ED & PLA \\
\hline Energy (kcal) & 200 & 0 \\
\hline Carbohydrate & $54 \mathrm{~g}$ & $0 \mathrm{~g}$ \\
\hline Caffeine & $160 \mathrm{mg}$ & $0 \mathrm{mg}$ \\
\hline Taurine & $2000 \mathrm{mg}$ & $0 \mathrm{mg}$ \\
\hline Panax Ginseng & $400 \mathrm{mg}$ & $0 \mathrm{mg}$ \\
\hline $\begin{array}{c}\text { Proprietary Energy Blend } \\
\text { (L-Carnitine, Guaraná, } \\
\text { Glucuronolactone) }\end{array}$ & $5000 \mathrm{mg}$ & $0 \mathrm{mg}$ \\
\hline
\end{tabular}


APPENDIX D: 35-KM COURSE DETAIL

\begin{tabular}{|c|c|c|c|}
\hline $\begin{array}{l}\text { Start point } \\
(\mathbf{k m})\end{array}$ & $\begin{array}{c}\text { Leg distance } \\
(\mathrm{km})\end{array}$ & $\begin{array}{c}\text { Total distance } \\
(\mathrm{km})\end{array}$ & Grade \\
\hline 0 & .745 & .745 & $\begin{array}{c}\text { Random between } \\
-3 \% \text { and } 3 \%\end{array}$ \\
\hline .745 & .005 & .75 & $0 \%$ \\
\hline .75 & .5 & 1.25 & $0 \%$ \\
\hline 1.25 & 3.495 & 4.745 & $\begin{array}{c}\text { Random between } \\
-3 \% \text { and } 3 \%\end{array}$ \\
\hline 4.745 & .005 & 4.75 & $0 \%$ \\
\hline 4.75 & 3.5 & 8.25 & $0 \%$ \\
\hline 8.25 & 3.495 & 11.745 & $\begin{array}{c}\text { Random between } \\
-3 \% \text { and } 3 \%\end{array}$ \\
\hline 11.745 & .005 & 11.75 & $5.4 \%$ \\
\hline 11.75 & .5 & 12.25 & $5.4 \%$ \\
\hline 12.25 & 3.495 & 15.745 & $\begin{array}{c}\text { Random between } \\
-3 \% \text { and } 3 \%\end{array}$ \\
\hline 15.745 & .005 & 15.75 & $0 \%$ \\
\hline 15.75 & 3.5 & 19.25 & $0 \%$ \\
\hline 19.25 & 3.495 & 22.745 & $\begin{array}{c}\text { Random between } \\
-3 \% \text { and } 3 \% \\
\end{array}$ \\
\hline 22.745 & .005 & 22.75 & $5.4 \%$ \\
\hline 22.75 & .5 & 23.25 & $5.4 \%$ \\
\hline 23.25 & 4.495 & 27.745 & $\begin{array}{c}\text { Random between } \\
-3 \% \text { and } 3 \%\end{array}$ \\
\hline 27.745 & .005 & 27.75 & $0 \%$ \\
\hline 27.75 & 3.5 & 31.25 & $0 \%$ \\
\hline 31.25 & 2.495 & 33.745 & $\begin{array}{c}\text { Random between } \\
-3 \% \text { and } 3 \%\end{array}$ \\
\hline 33.745 & .005 & 33.75 & $5.4 \%$ \\
\hline 33.75 & .5 & 34.25 & $5.4 \%$ \\
\hline 34.25 & .75 & 35 & $\begin{array}{c}\text { Random between } \\
-3 \% \text { and } 3 \%\end{array}$ \\
\hline
\end{tabular}




\section{APPENDIX E: FIGURES OF RESULTS}

Figures appear in this section to give a visual representation of data. When a significant interaction or main effect was found, post-hoc analysis was utilized using the LSD method. Treatment-by-time interactions are indicated with an asterisk $(*)$, main effects for treatment when no interaction was found are indicated by an ampersand $\left({ }^{\&}\right)$ and main effects for time when no interaction was found are indicated by different letters $\left({ }^{A-G}\right)$.

\section{Physical Performance}

Figure 1: Race Performance by Treatment

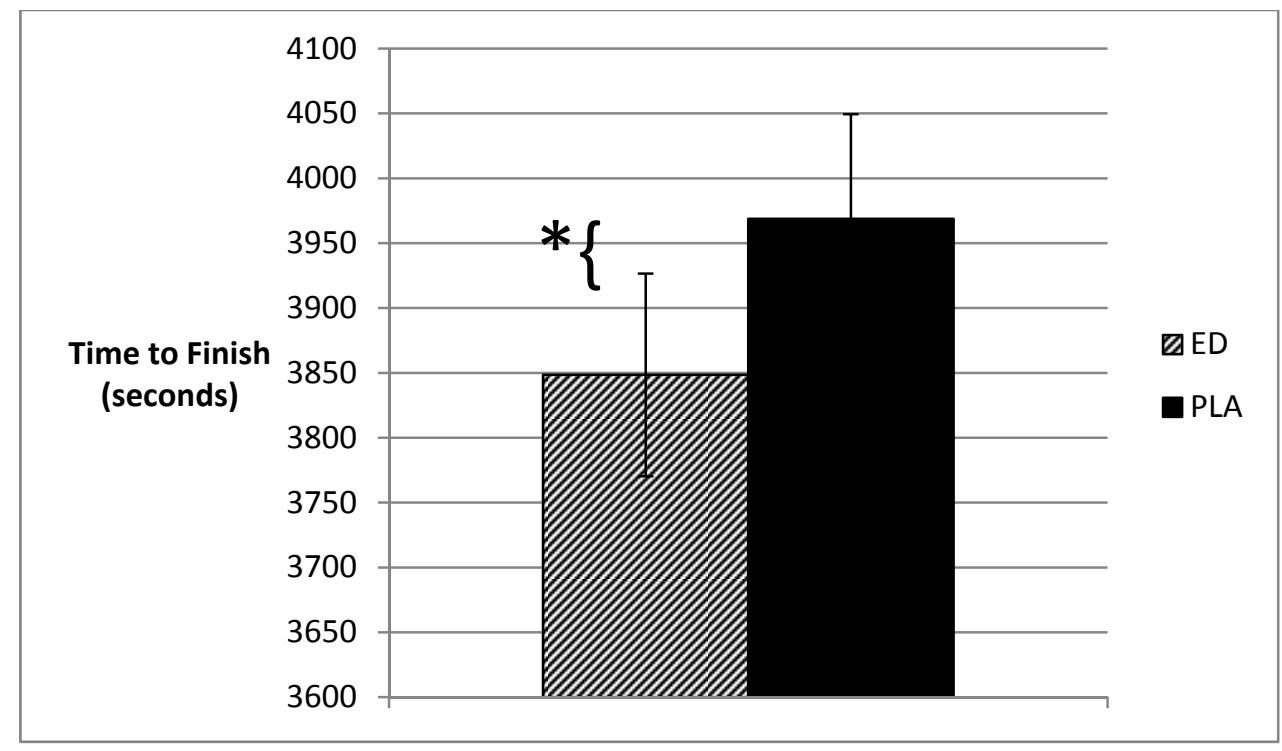

*ED resulted in significant faster time to finish. 
Figure 2: Rating of Perceived Exertion

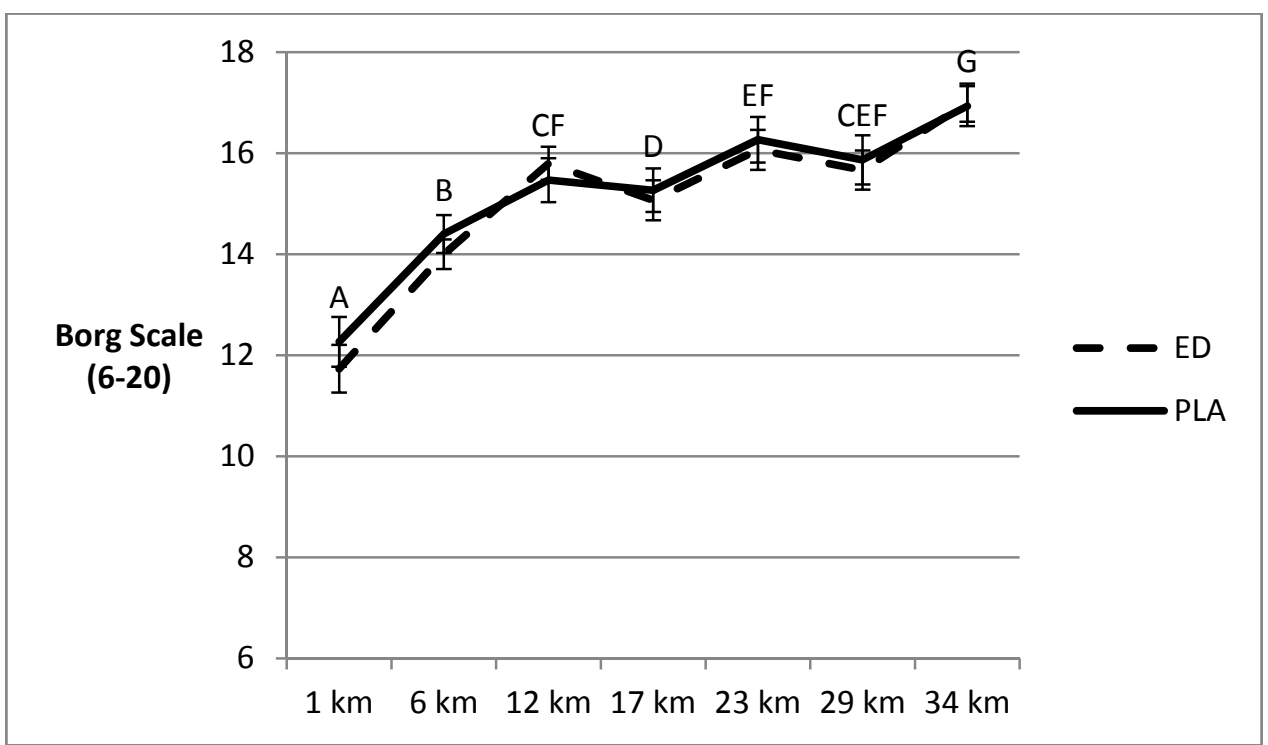

${ }^{\mathrm{A}-\mathrm{G}}$ Significant differences in times. Participants were riding up a simulated hill at 12 $\mathrm{km}, 23 \mathrm{~km}$, and $34 \mathrm{~km}$ which resulted in peaks in RPE at these times.

\section{Cognitive and Psychomotor Performance}

Figure 3: Tap Test, Percent of Precise Responses

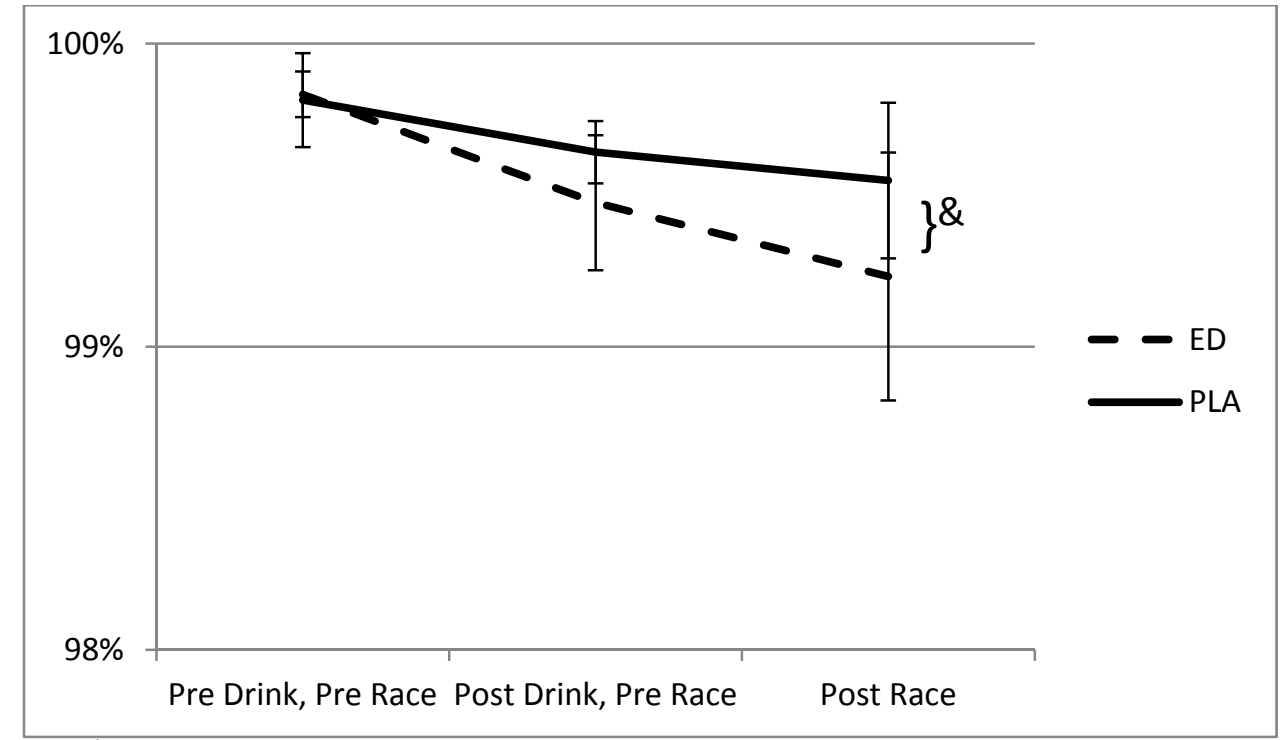

${ }^{\&}$ Main effect of treatment was found. 
Figure 4: Executive Function Test, Total Time

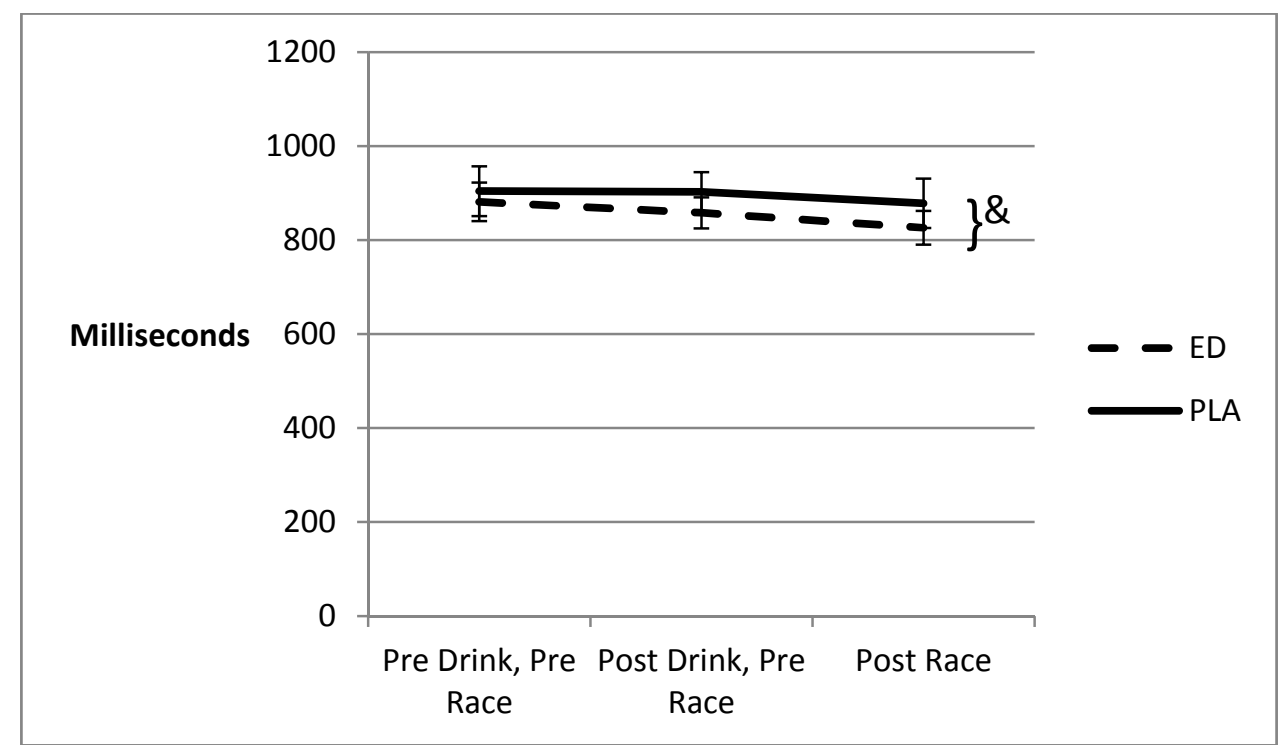

${ }^{\&}$ Main effect for treatment was found. The effect is probably due to confounds which caused participants to perform the task faster on the day they received ED and not due to the treatment since no time by treatment interaction was found.

Figure 5: Stroop Test, Reaction Time to All Stimuli

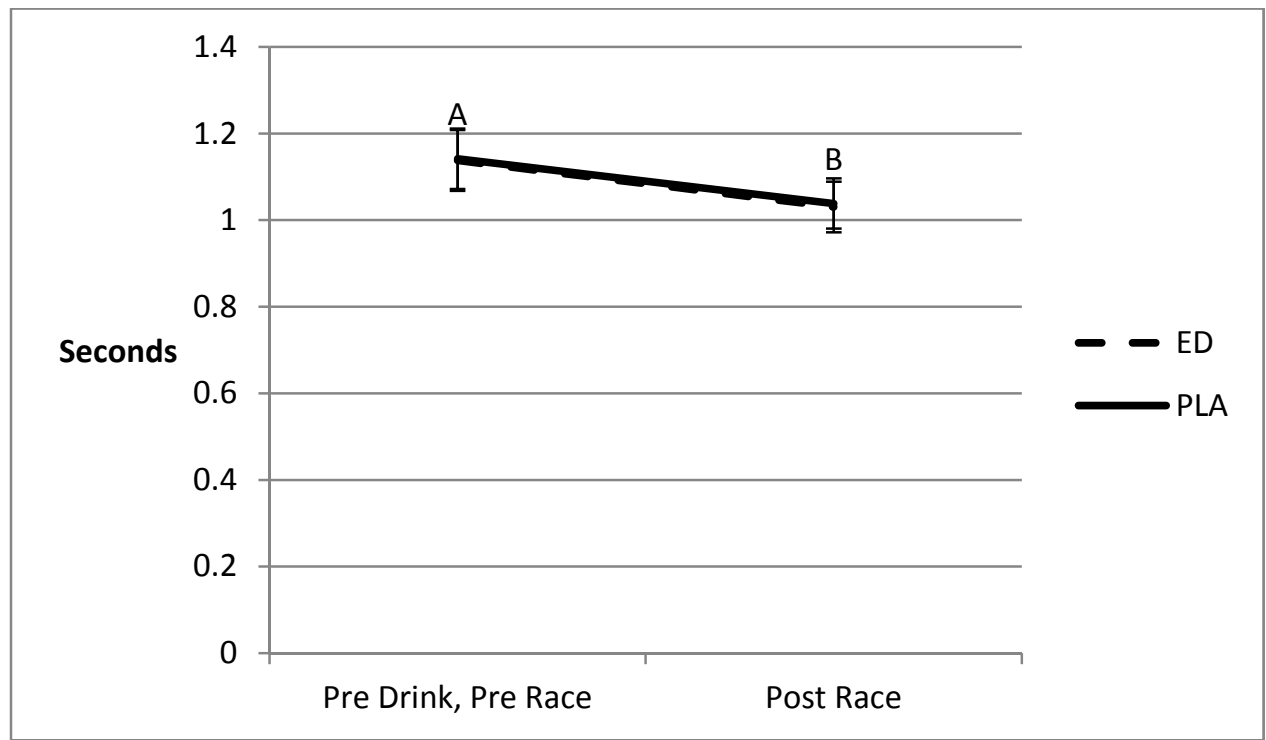

${ }^{\mathrm{A}, \mathrm{B}}$ Main effect of time. The data indicate that participants reacted faster regardless of stimulus type while exercising at $70 \%$ of $\mathrm{VO}_{2 \max }$ immediately after completing the 35-km time-trial race. 
Figure 6: Stroop Test, Reaction Time to Incongruent Stimuli

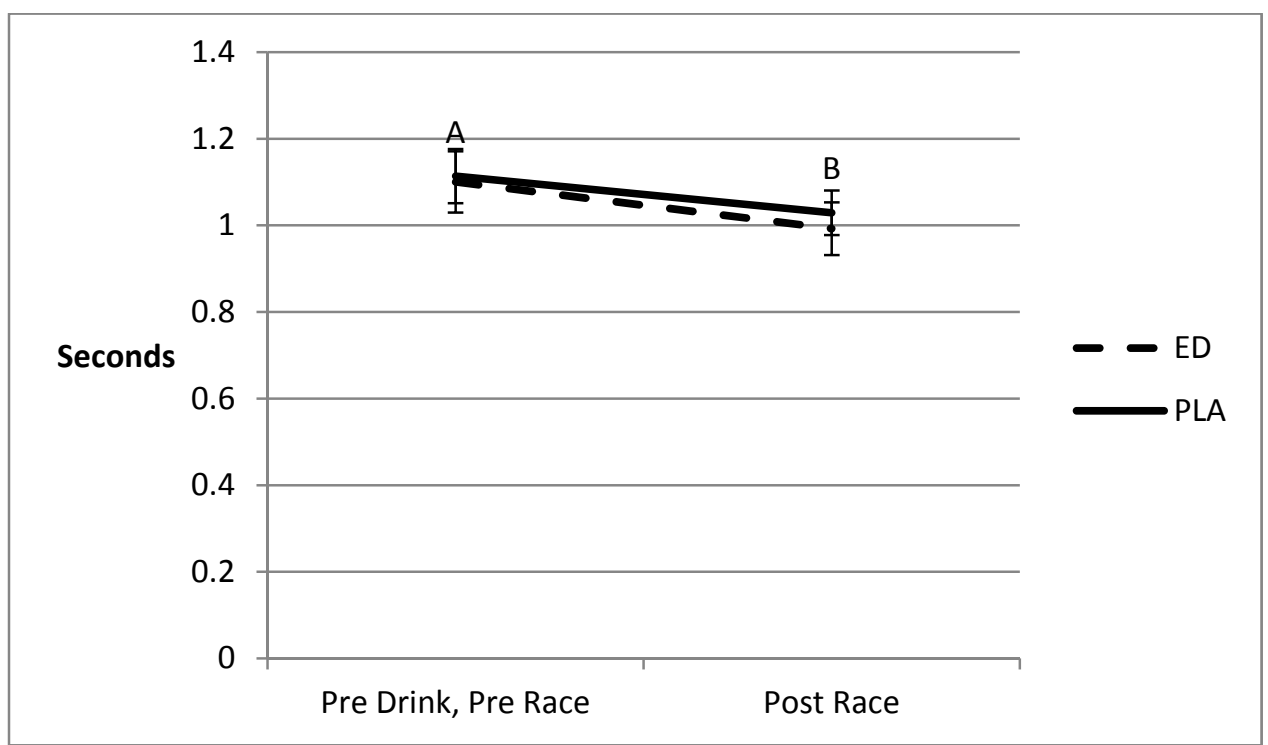

${ }^{\mathrm{A}, \mathrm{B}}$ Main effect of time when presented with "incongruent" stimuli.

Figure 7: Tap Test, Simple Movement Time

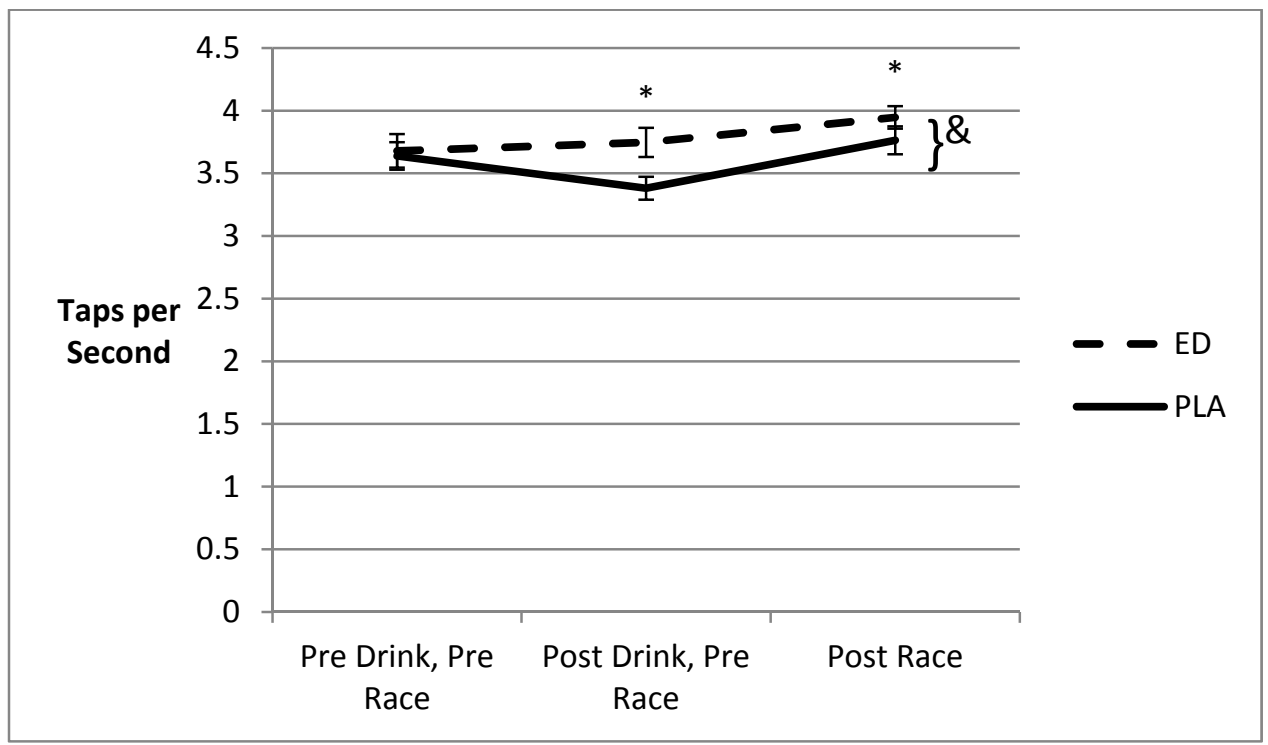

*Treatment-by-time interaction. ED resulted in increased taps per second prior to exercise onset as well as after completion of the $35-\mathrm{km}$ time trial. Conclusions can be made about ED since participants performed at the same level prior to drinking. ${ }^{\&}$ Main effect for treatment was also found. 
Figure 8: Executive Function Test, Simple Movement

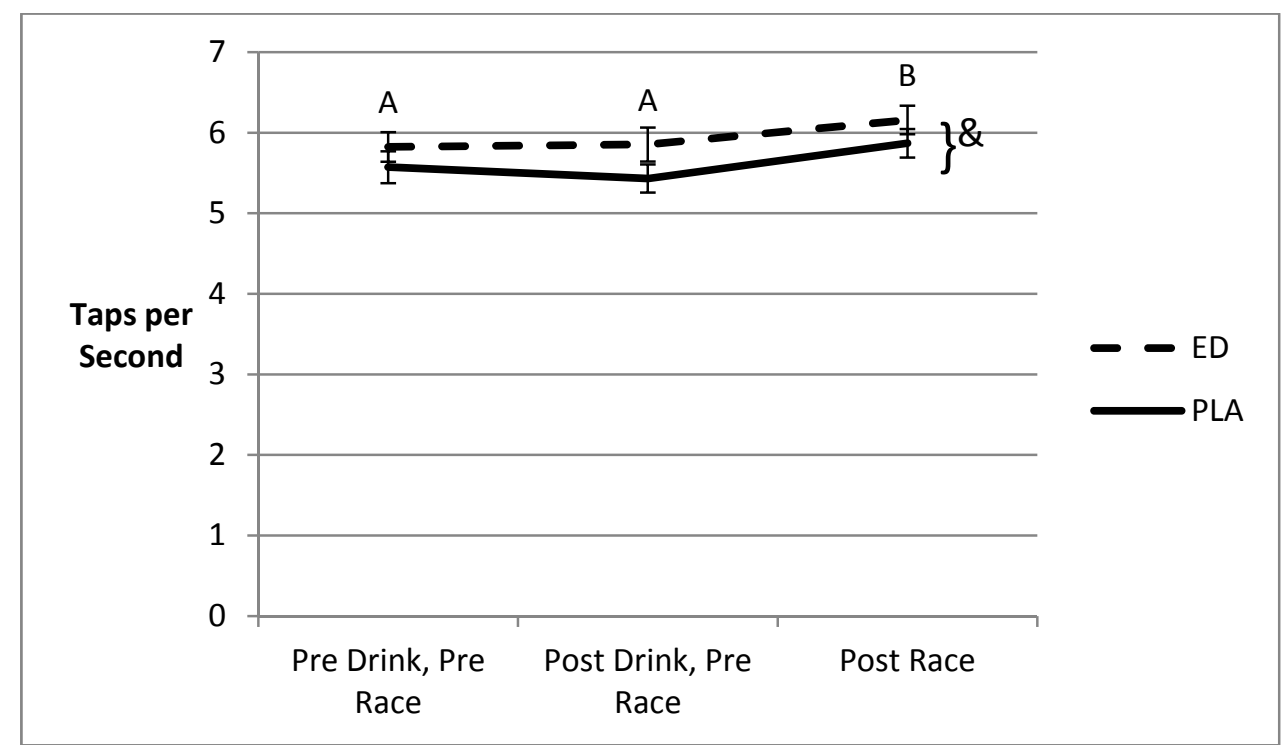

${ }^{\&}$ Main effect for treatment. Similar to Executive Function Total Time (\&Main effect for treatment was found. The effect is probably due to confounds which caused participants to perform the task faster on the day they received ED and not due to the treatment since no time by treatment interaction was found.), the main effect for treatment is likely due to uncontrolled factors. ${ }^{A, B}$ Main effect for time indicating faster movement time after exercise.

Figure 9: Executive Function Test, Gross Movement Time

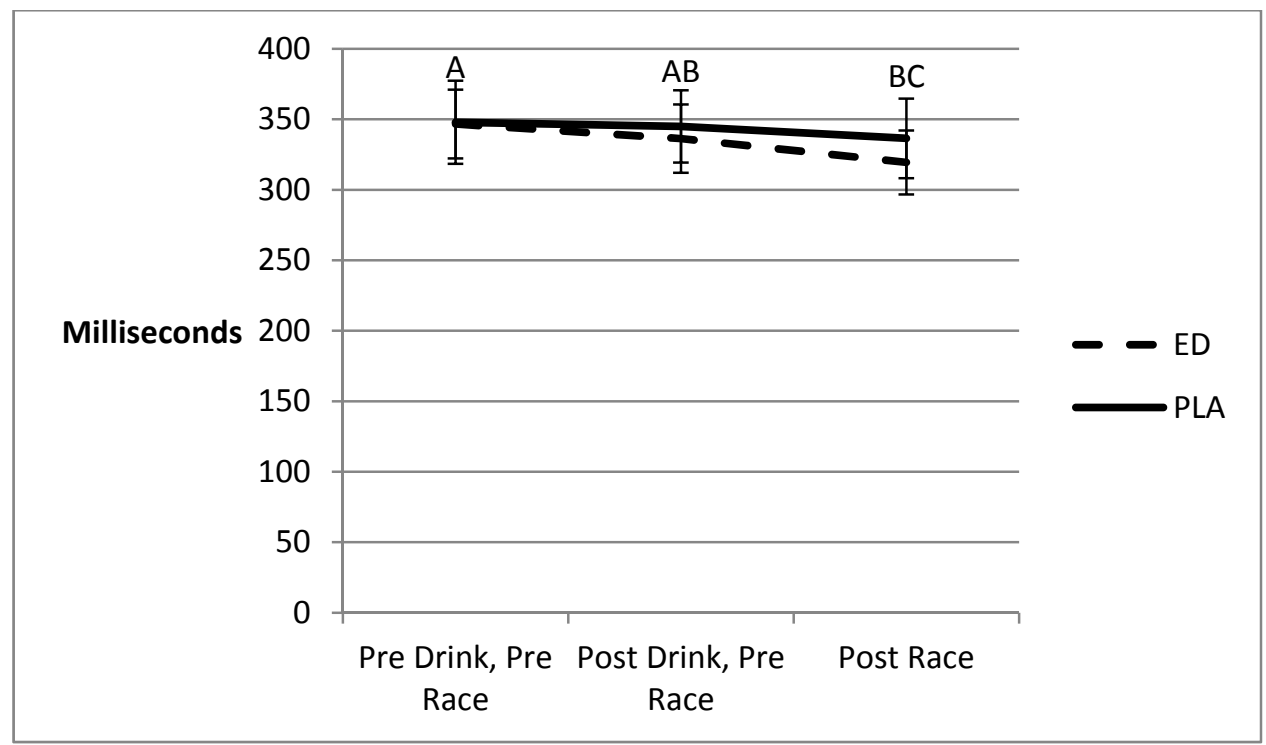

${ }^{A-C}$ Main effect for time. Gross movement during the executive function test was faster after exercise. 
Figure 10: Reaction Time Test, Gross Movement Time when Correct

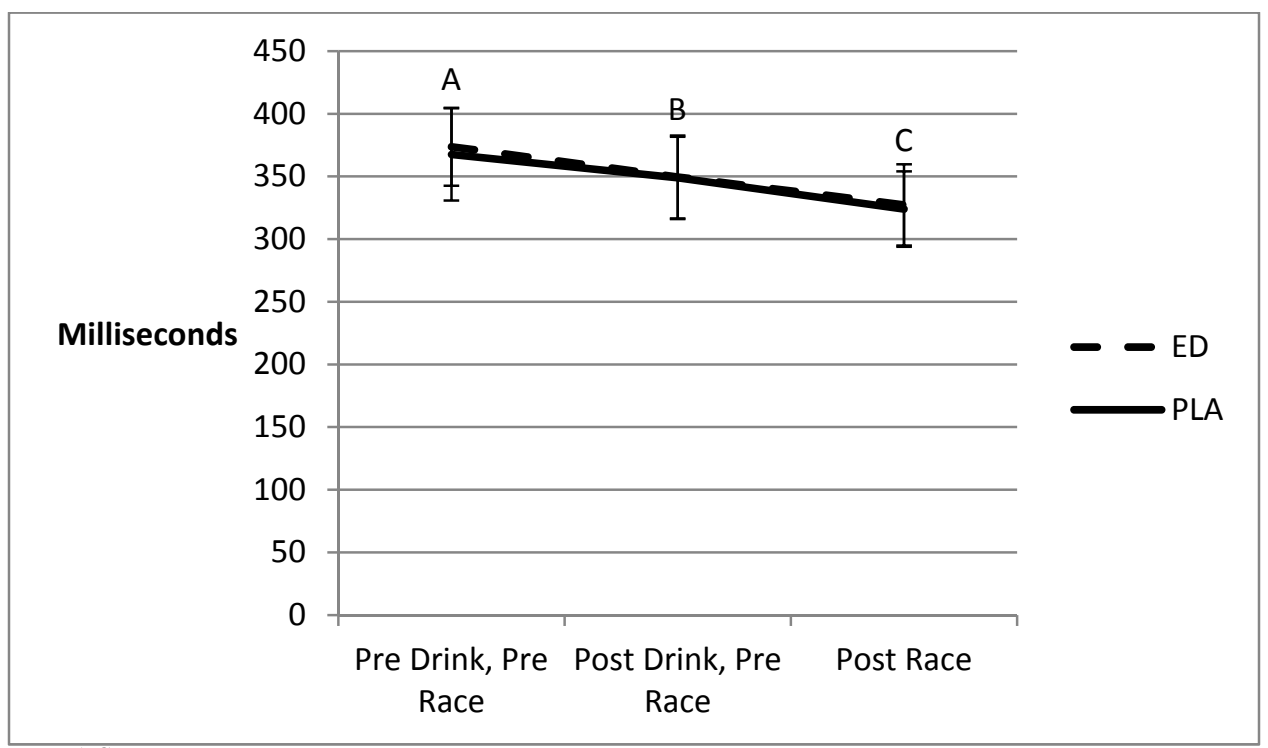

${ }^{A-C}$ Main effect of time. Gross movement during the reactive time test was faster after exercise.

\section{Metabolic Effects}

Figure 11: Insulin

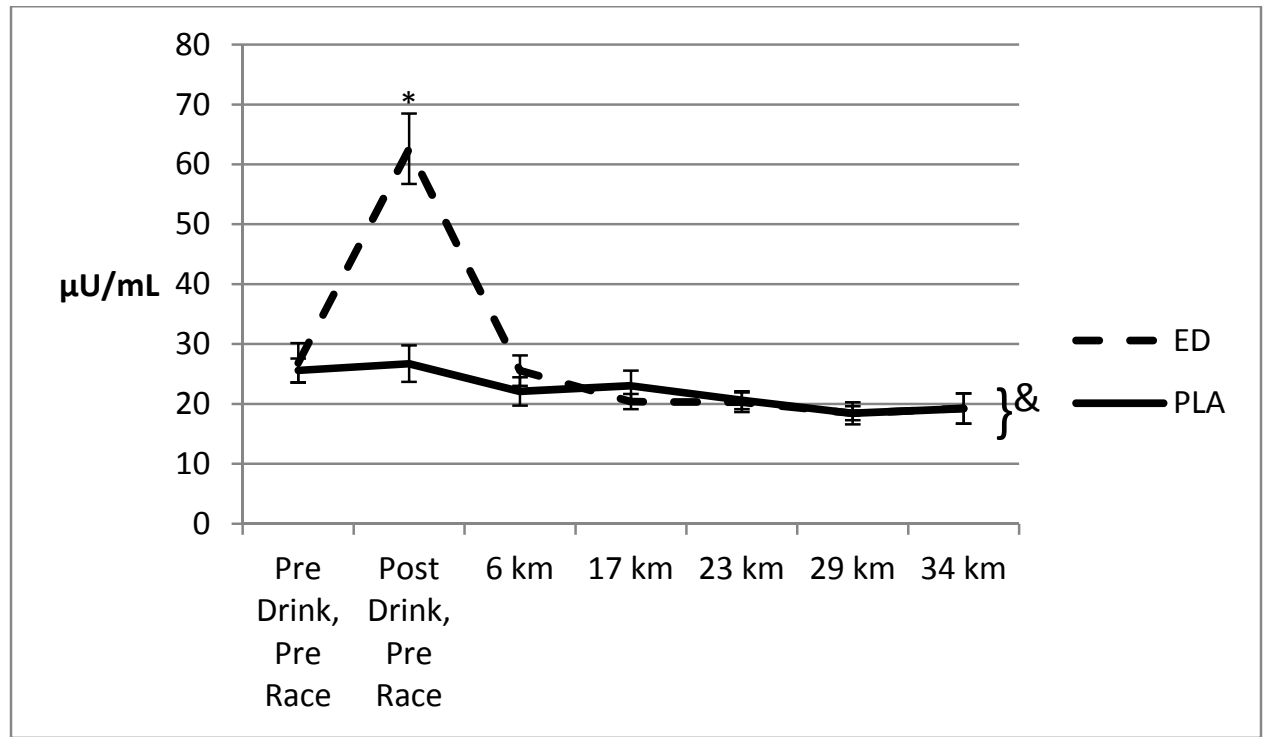

*Treatment-by-time interaction. Insulin spiked when participants received ED but returned to PLA levels by $6 \mathrm{~km}$. \& Main effect for treatment was also found. 
Figure 12: Glucose

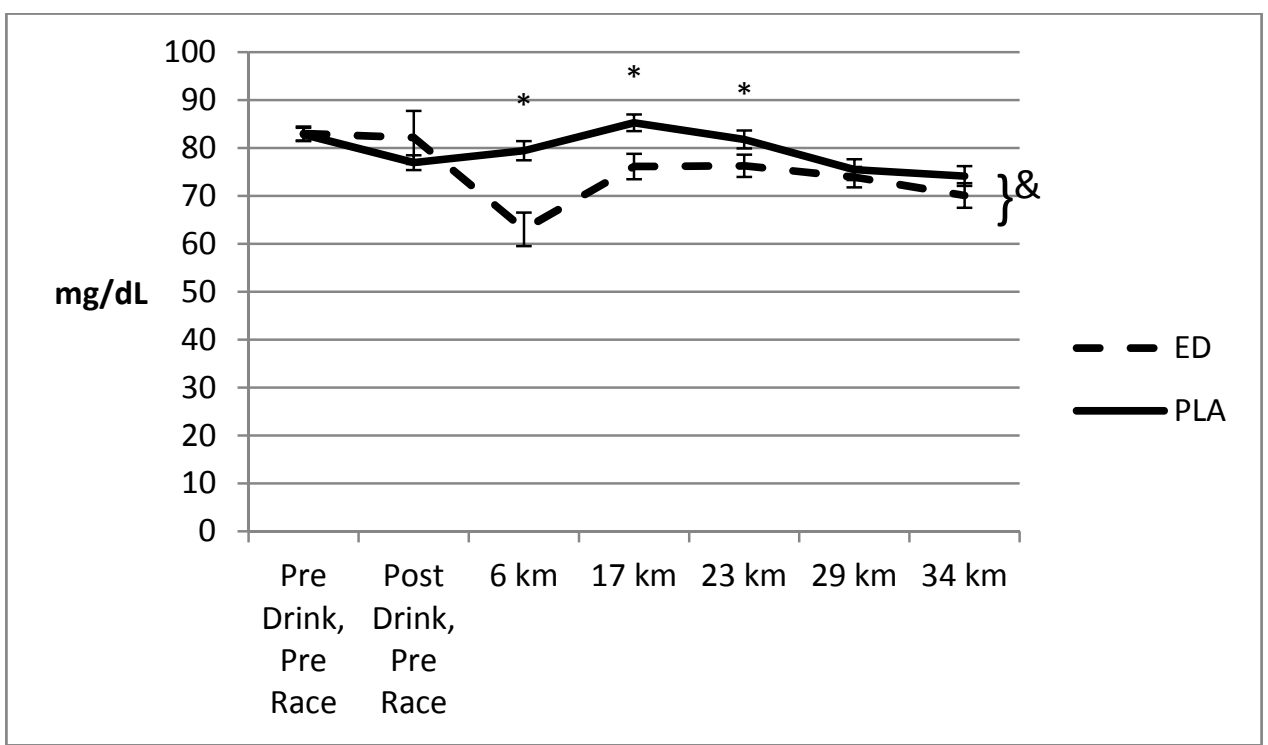

*Treatment-by-time interaction. Glucose is maintained by the insulin spike so that there is no difference between ED and PLA before the race starts. When the race starts, glucose fell in ED due to the lasting effects of insulin, though there is no difference by $23 \mathrm{~km} .{ }^{\&}$ Main effect for treatment was also found.

Figure 13: Free Fatty Acids

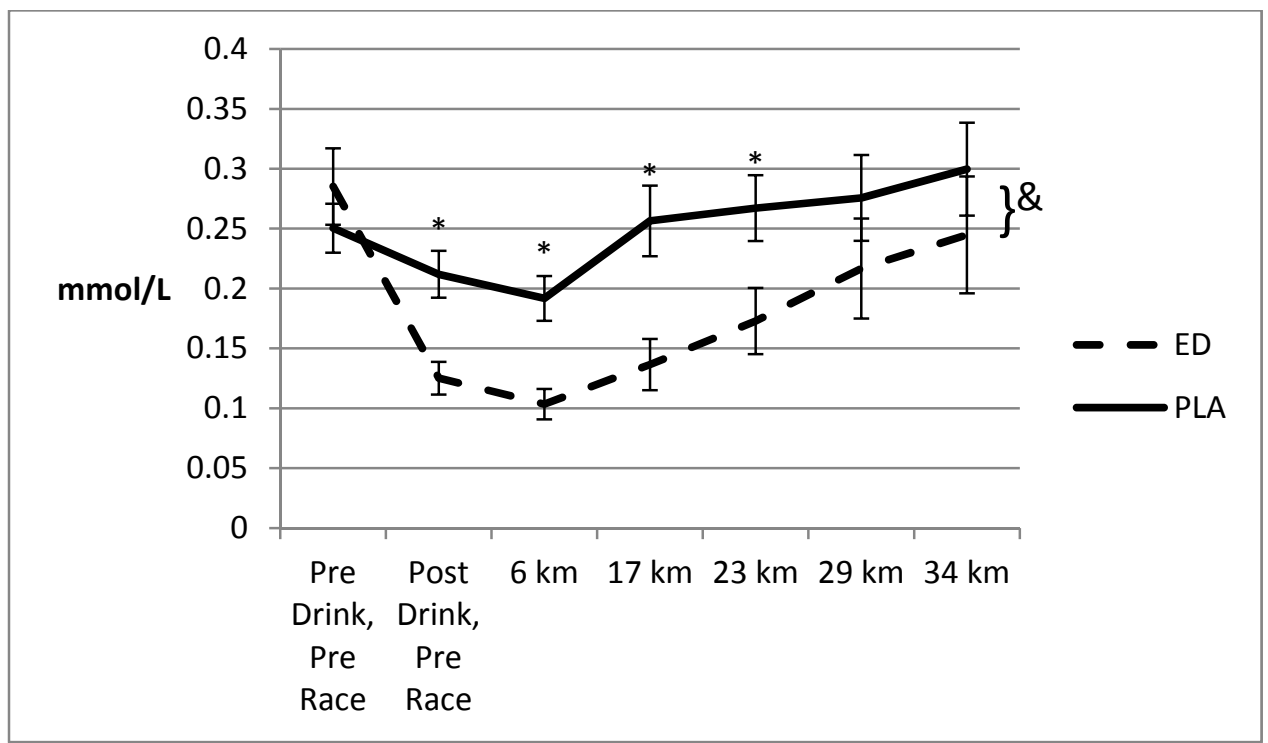

Figure $7 *$ Treatment-by-time. Free fatty acids fell after the insulin spike induced by ED, but the exercise stimulus increased lipolysis. By $29 \mathrm{~km}$ there is no difference between ED and PLA. ${ }^{\&}$ Main effect for treatment was also found. 
Figure 14: Glycerol

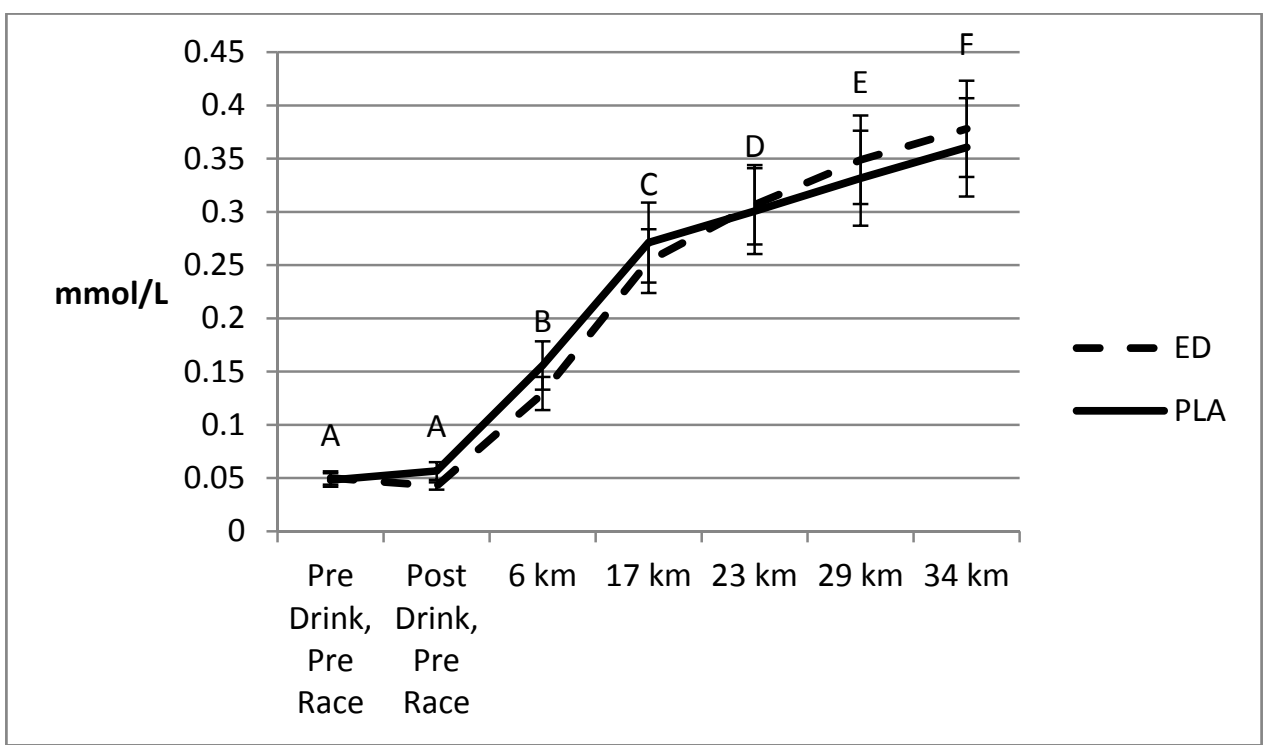

${ }^{A-F}$ Main effect of time. At rest there is no change in glycerol, but over the course of the $35-\mathrm{km}$ race increased lipolysis causes increased blood glycerol.

Figure 15: Caffeine

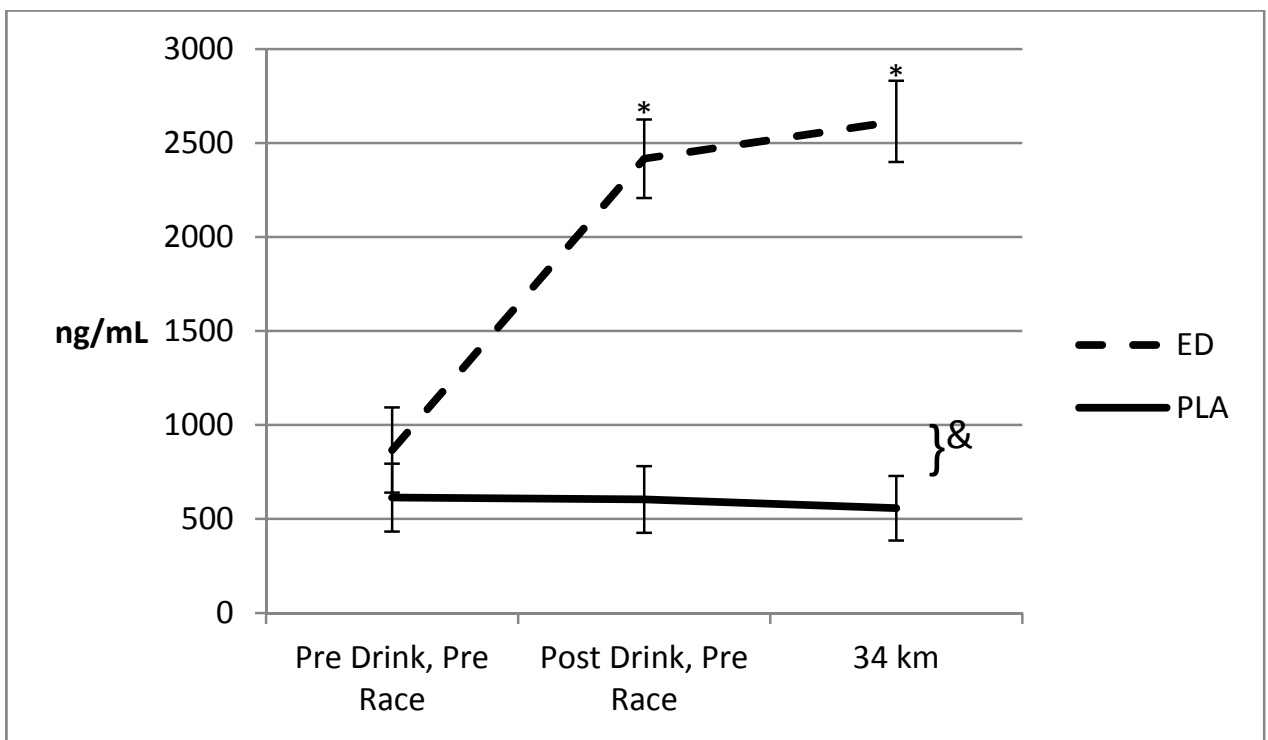

*Treatment-by-time interaction. ED elevated caffeine throughout the race. ${ }^{\&}$ Main effect for treatment was also found. 
Figure 16: Epinephrine

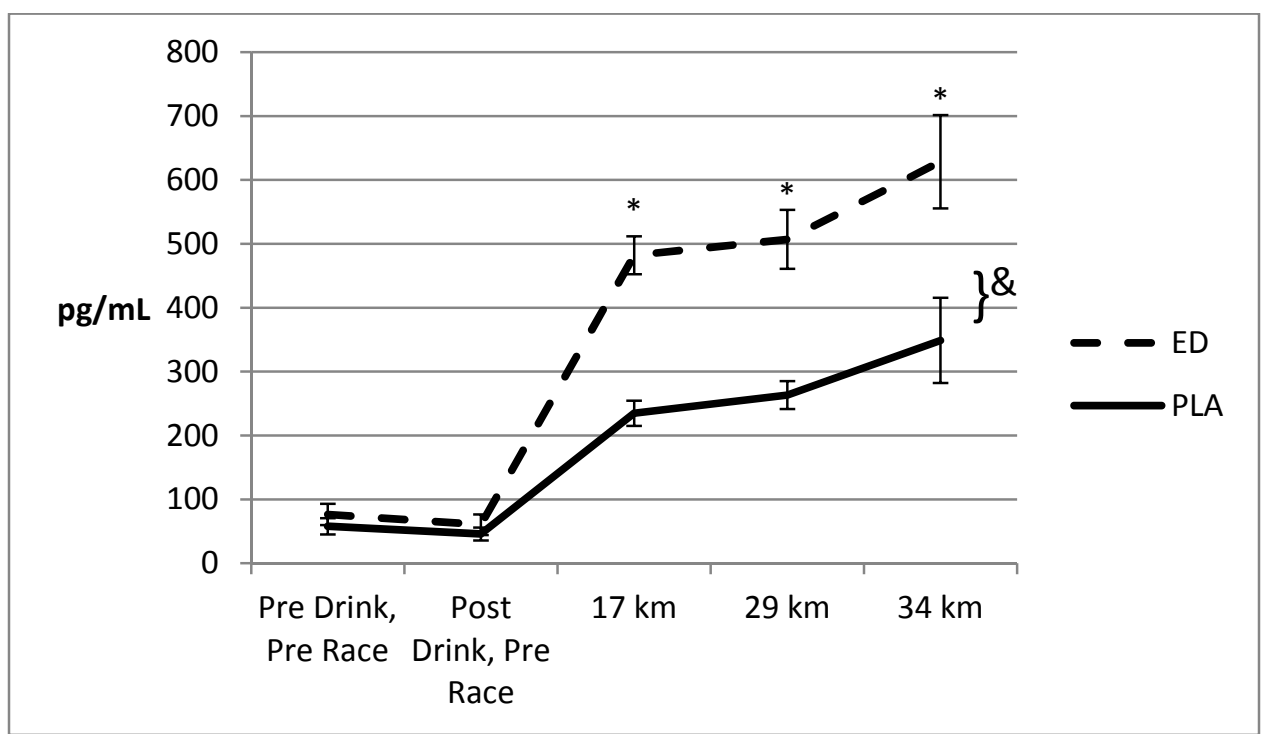

*Treatment-by-time interaction. ED elevated epinephrine levels over PLA once exercise started. These levels remained elevated throughout exercise. ${ }^{\&}$ Main effect for treatment was also found.

Figure 17: Norepinephrine

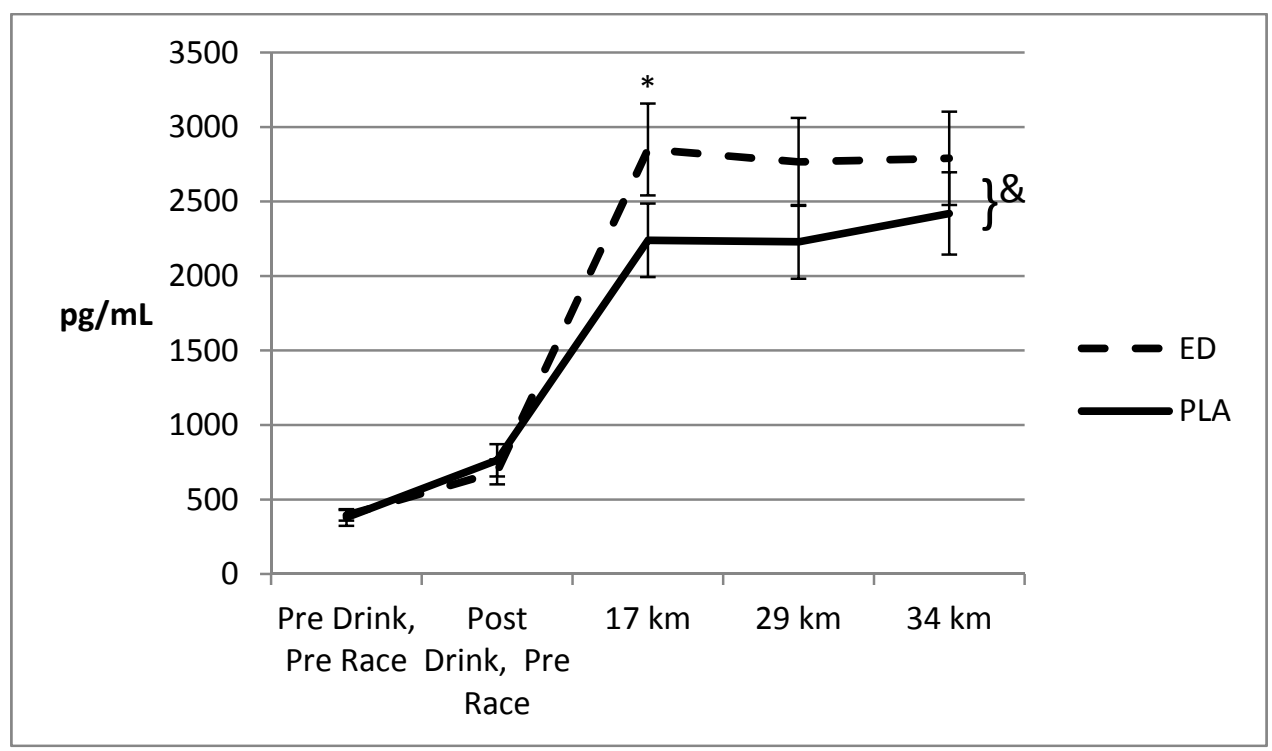

*Treatment-by-time interaction. ED caused norepinephrine to be elevated at $17 \mathbf{~ k m}$ more than PLA, though there were no differences through the end of the trial. ${ }^{\&}$ Main effect for treatment was also found. 
Figure 18: $\beta$-Endorphins

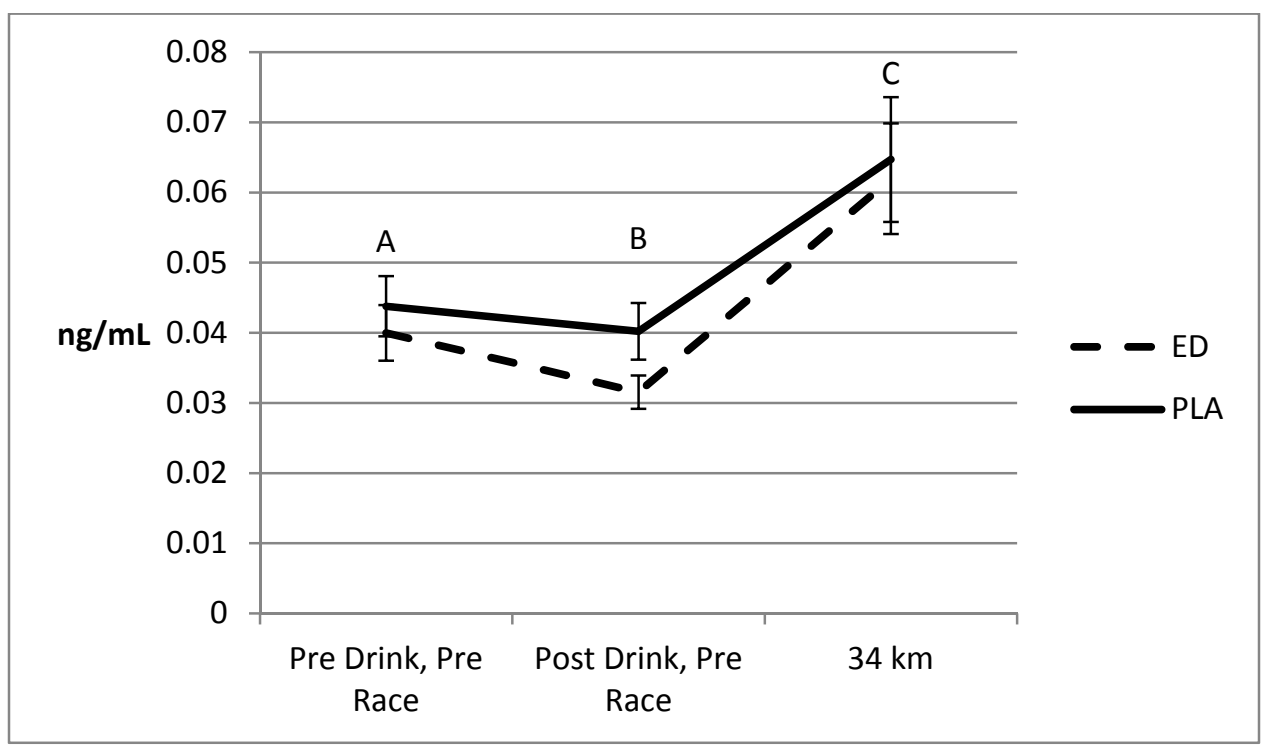

${ }^{A-C}$ Main effect of time. $\beta$-endorphins fell while participants were resting before exercise but the rose throughout the race.

Figure 19: Cortisol

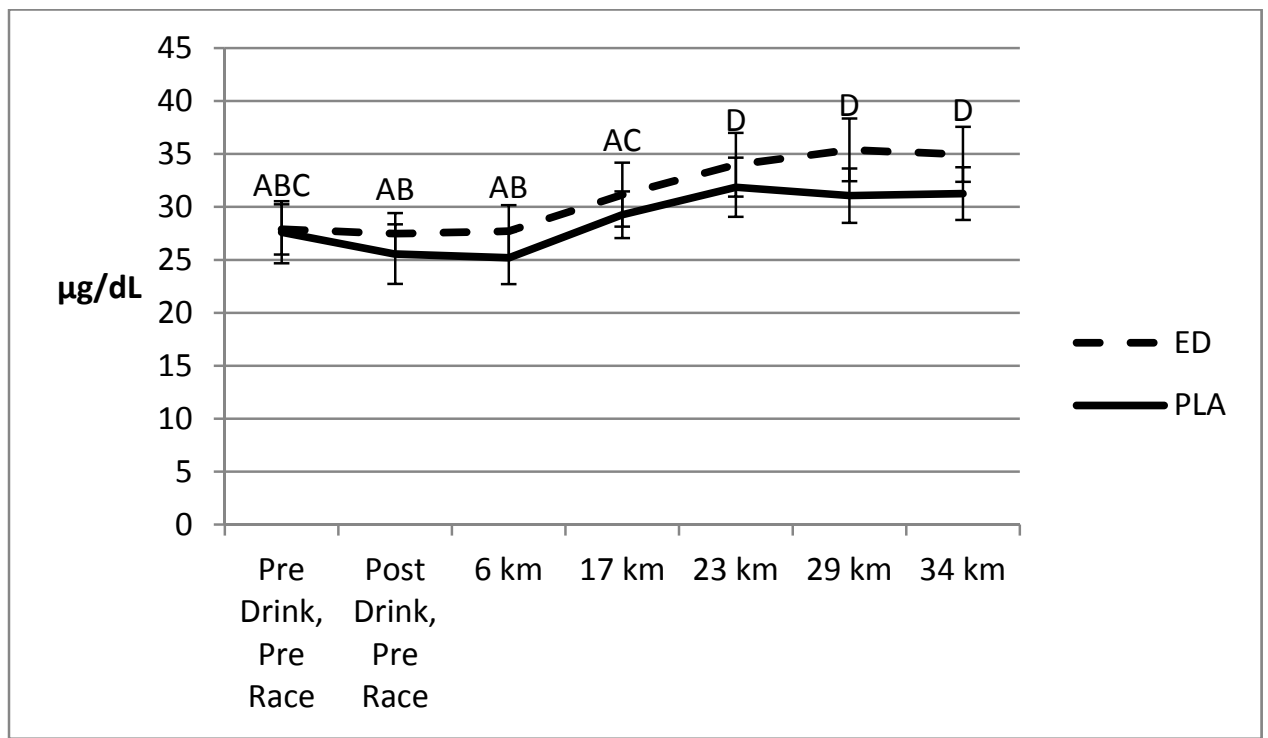

${ }^{\text {A-D }}$ Main effect of time. Cortisol rose due to exercise by $23 \mathrm{~km}$ and remained elevated through the end of the race. 
Figure 20: Lactate

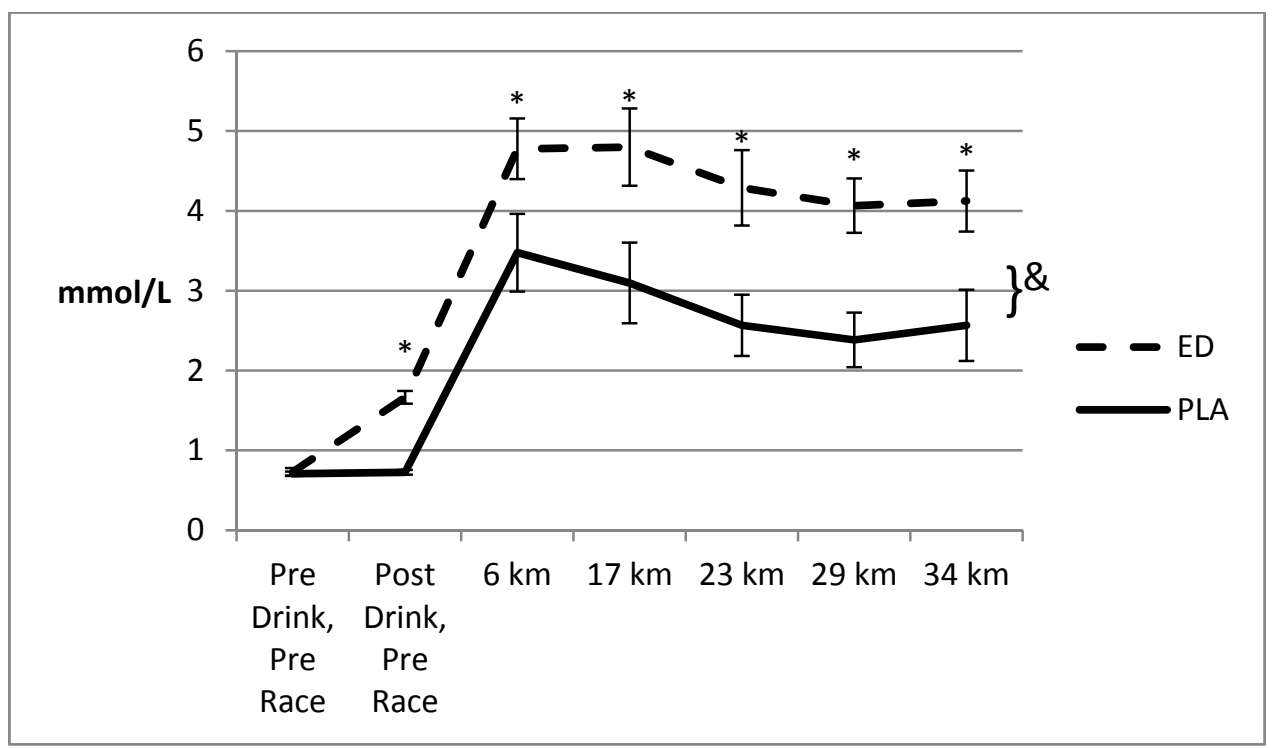

*Treatment-by-time interaction. ED increased lactate more than PLA starting at rest and continuing throughout the exercise trial. ${ }^{\&}$ Main effect for treatment.

Figure 21: Myoglobin

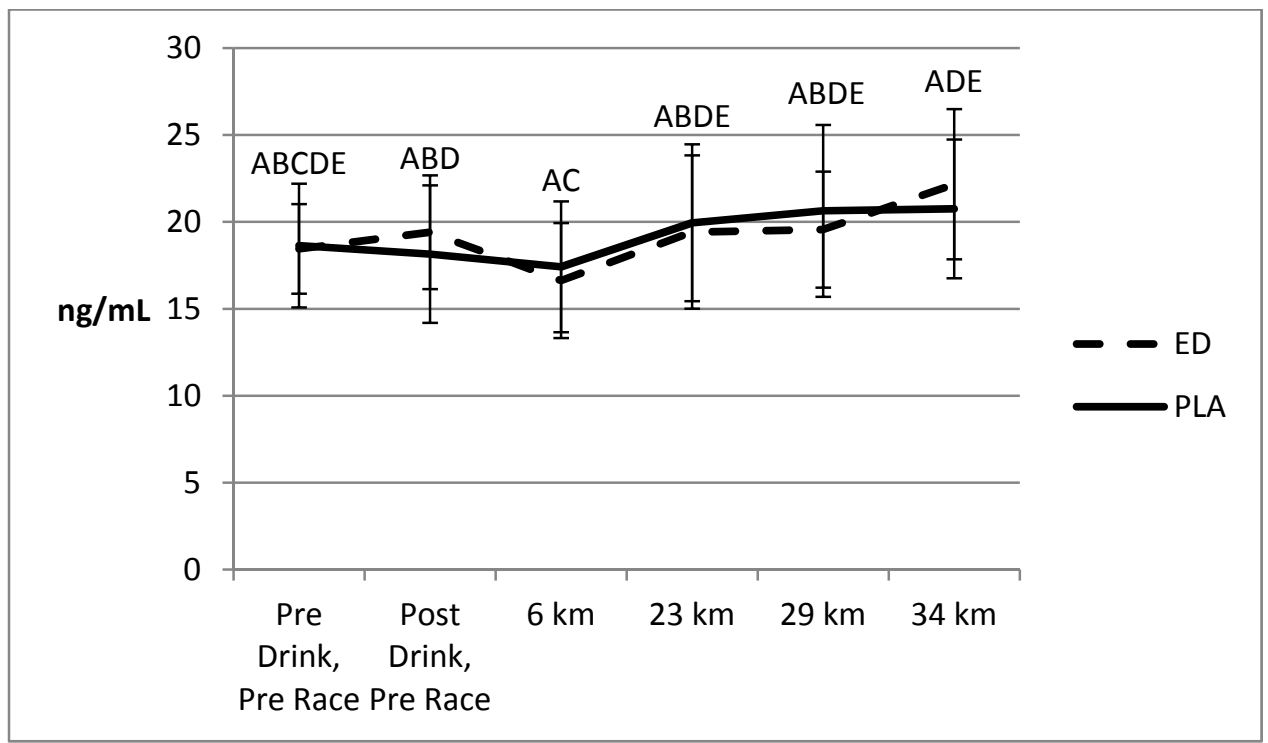

${ }^{\mathrm{A}-\mathrm{E}}$ Main effect for time. While there is slight variation from time to time, myoglobin did not significantly differ from baseline at any time point. 
Figure 22: $\mathrm{VO}_{2}$

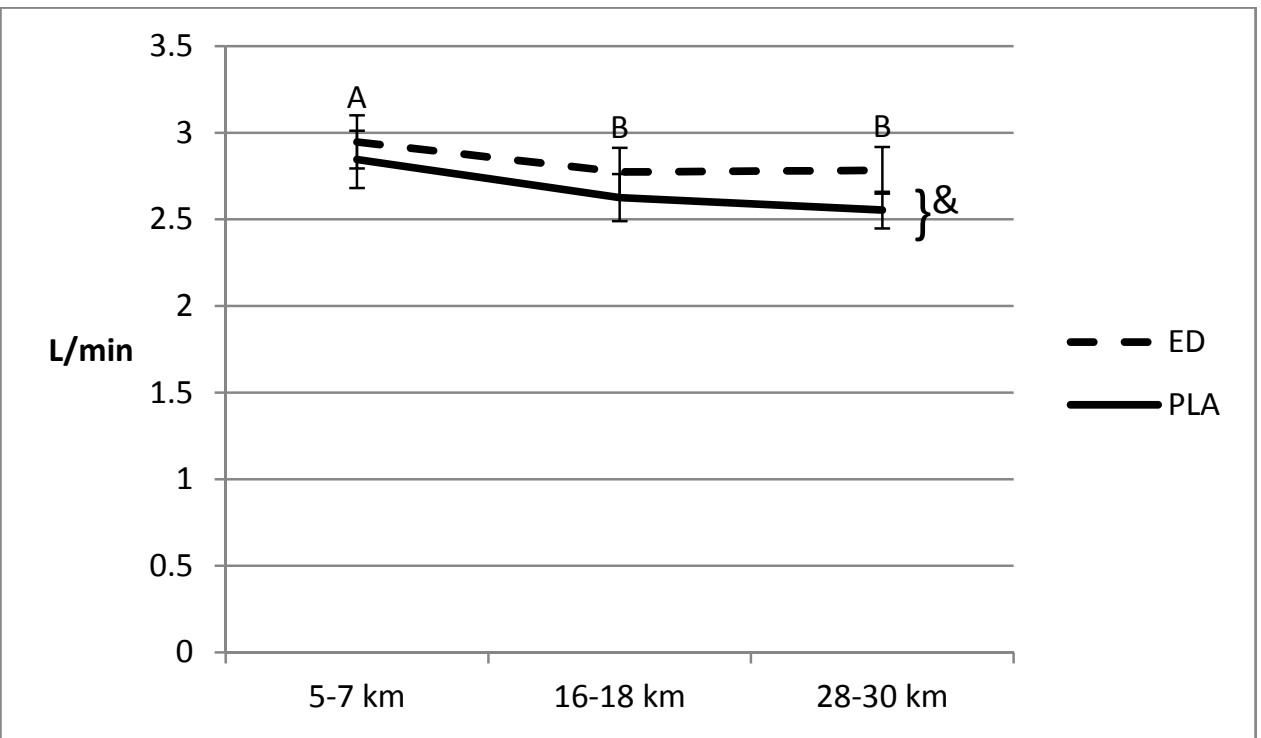

${ }^{\&}$ Main effect for treatment. ${ }^{\mathrm{A}, \mathrm{B}}$ Main effect for time. ED resulted in greater oxygen consumption throughout the ride though VO2 fell as exercise progressed regardless of drink provided.

Figure 23: Respiratory Exchange Ratio

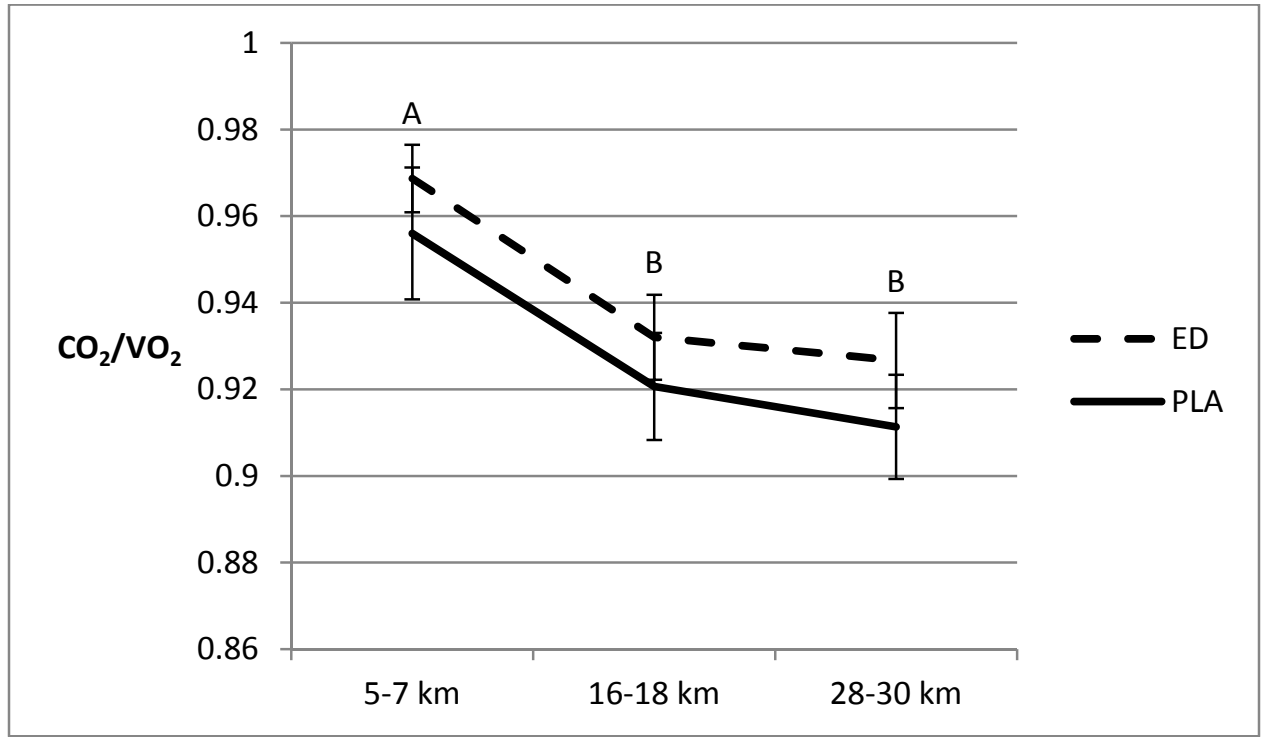

${ }^{\mathrm{A}, \mathrm{B}}$ Main effect for time. The respiratory exchange ratio fell throughout the ride. 
Figure 24: Heart Rate

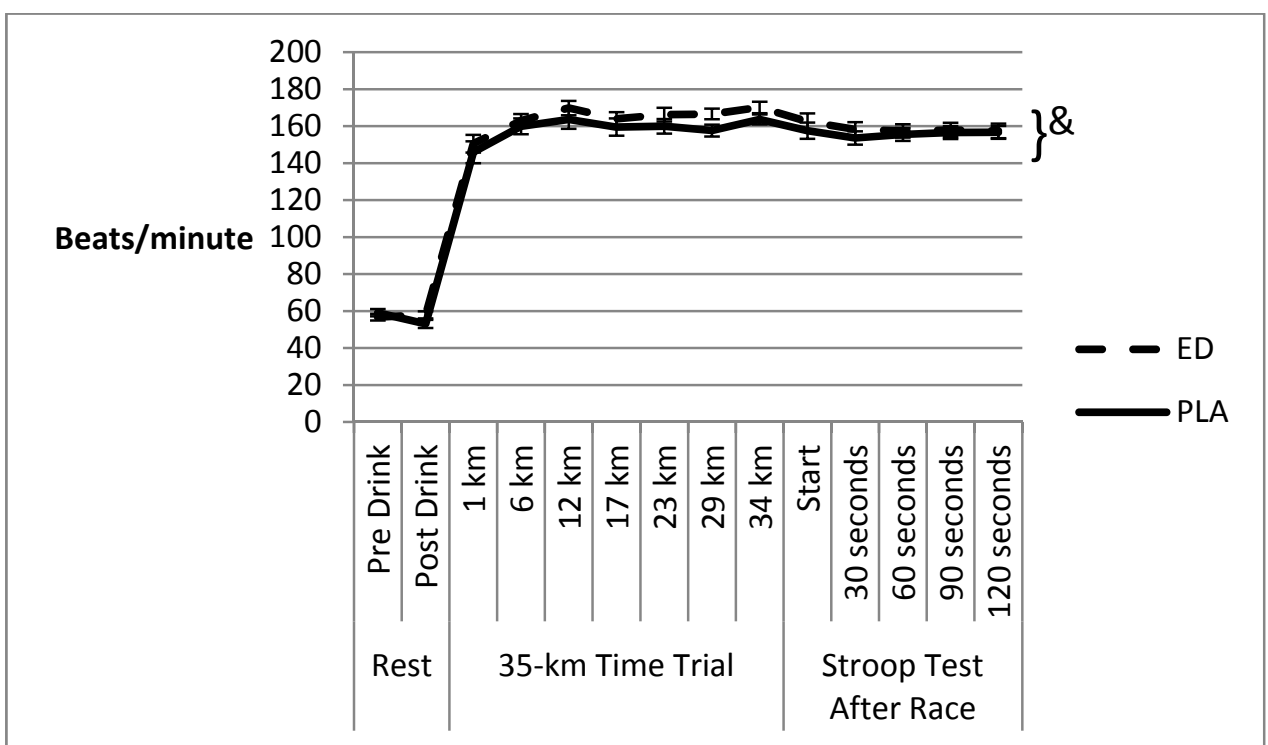

${ }^{\&}$ Main effect for treatment and time. 


\section{APPENDIX F: FORMULAS}

\section{Equation 1: Estimating a Missing Data Point from an Otherwise} Complete Matrix

$$
\begin{gathered}
x=\frac{t T+r R-S}{(t-1)(r-1)} \\
x=\text { missing data point } \\
t=\text { number of treatments }
\end{gathered}
$$

$T=$ sum of scores for treatment in which the missing data point $(x)$ occurs

$$
r=\text { number of rows }
$$

$R=$ sum of scores for row in which the missing data point $(x)$ occurs

$$
S=\text { sum of all observed values }
$$

\section{AN EXAMPLe:}

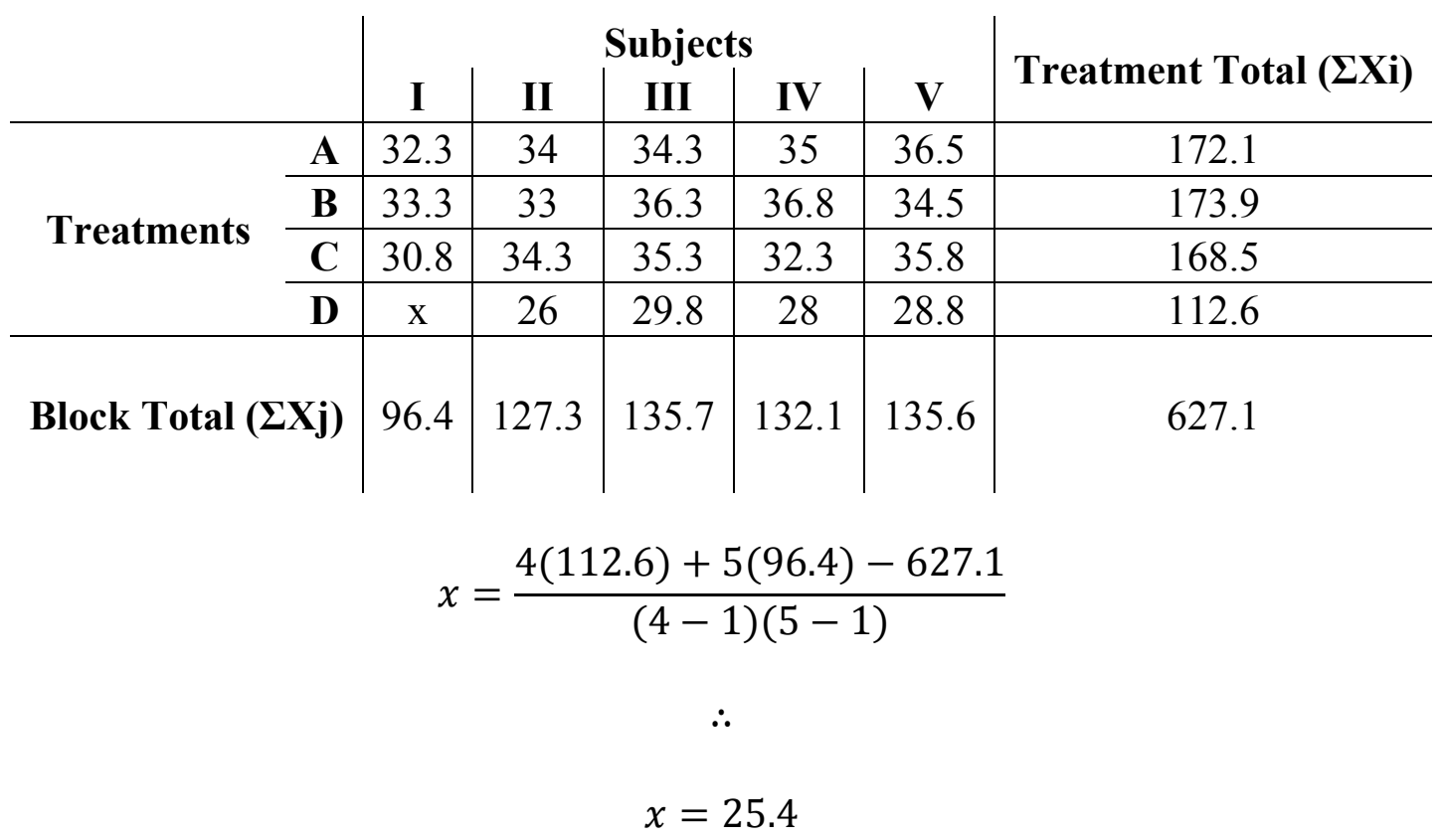




\section{Equation 2: Estimating Maximal Heart Rate}

$$
\begin{gathered}
H R_{M A X}=208-(A \times 0.7) \\
H R_{M A X}=\text { maximal heart rate } \\
A=\text { Age in years }
\end{gathered}
$$

An Example for a 30 Year Old Participant:

$$
\begin{gathered}
H R_{\text {MAX }}=208-(30 \times 0.7) \\
\therefore \\
H R_{M A X}=187
\end{gathered}
$$

Equation 3: Calculating Work Rate to Elicit a Specific Percentage of

$$
\begin{gathered}
\mathbf{V O}_{2 \mathbf{M A X}} \\
W=V O_{2 M A X} \times P-300 / 12.5 \\
W=\text { Work Rate in Watts } \\
V O_{2 M A X}=\text { Maximal oxygen consumption } \mathrm{in} \mathrm{ml} / \mathrm{min} \\
P=\text { Percentage of maximal oxygen consumption sought }
\end{gathered}
$$

An Example for Eliciting $50 \%$ OF VO 2 Max IN A PARTICIPANT With VO2MAX OF 4100 ML/MIN :

$$
\begin{gathered}
W=4100 \times 0.5-300 / 12.5 \\
\therefore \\
W=140
\end{gathered}
$$


APPENDIX G: EXERCISE VISIT TIMELINE

Timeline for Experimental Rides
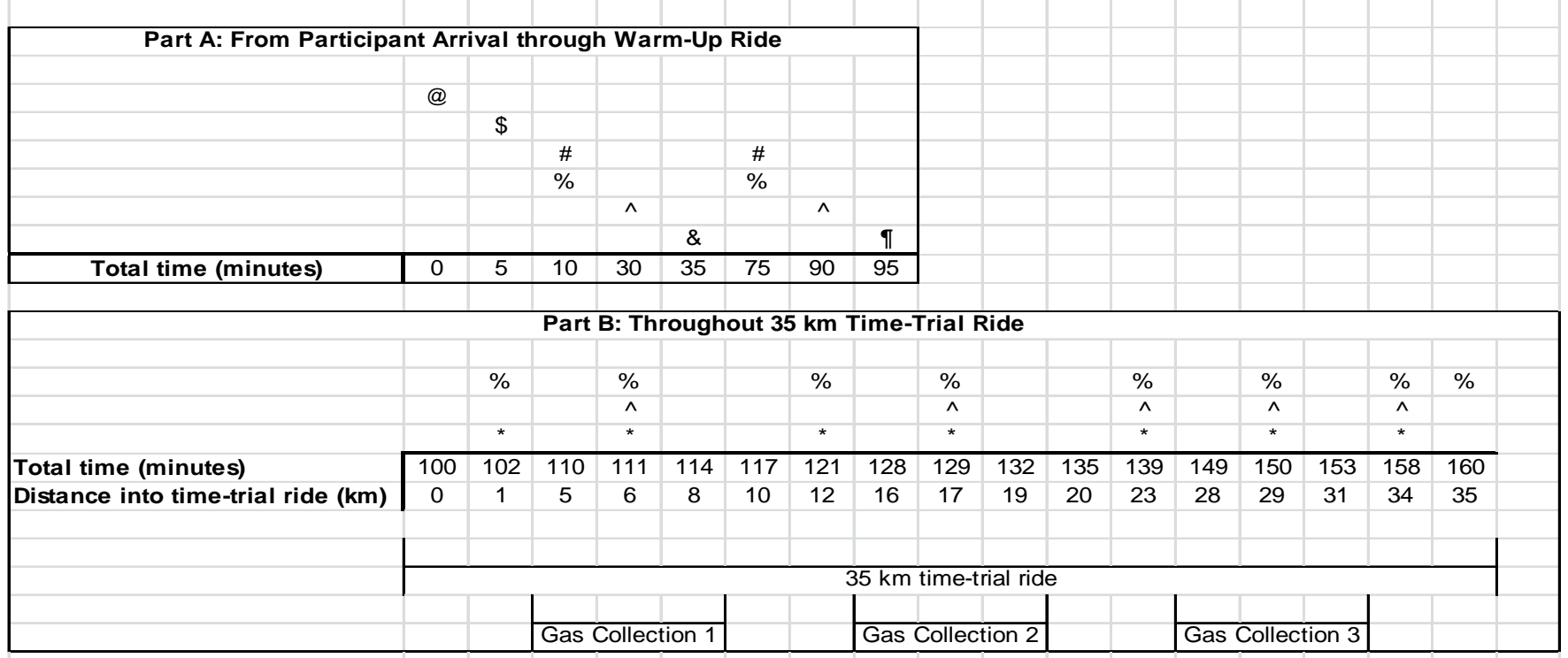

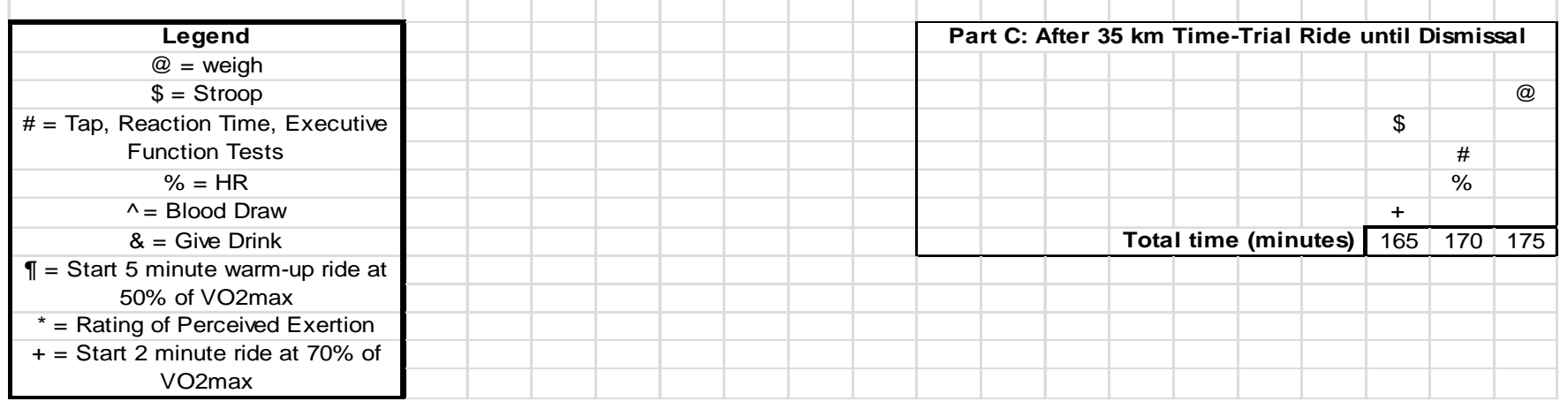




\section{REFERENCES}

Abebe, W., \& Mozaffari, M. S. (2011). Role of taurine in the vasculature: an overview of experimental and human studies. American Journal of Cardiovascular Diseases, 1(3), 293-311.

Adan, A., \& Serra-Grabulosa, J. M. (2010). Effects of caffeine and glucose, alone and combined, on cognitive performance. Human Psychopharmacology: Clinical and Experimental, 25(4), 310-317. Wiley Online Library. doi:10.1002/hup

Alford, C., Cox, H., \& Wescott, R. (2001). The effects of Red Bull Energy Drink on human performance and mood. Amino Acids, 21(2), 139-150.

Alles, G. A., \& Feigen, G. A. (1942). The influence of benzedrine on work-decrement and patellar reflex. American Journal Of Physiology, (136), 392-400.

Babu, K. M., Church, R. J., \& Lewander, W. L. (2008). Energy Drinks: The New EyeOpener For Adolescents. Clinical Pediatric Emergency Medicine, 9(1), 35-42. doi:10.1016/j.cpem.2007.12.002

Bell, D. G., \& McLellan, T. M. (2002). Exercise endurance 1, 3, and $6 \mathrm{~h}$ after caffeine ingestion in caffeine users and nonusers. Journal of Applied Physiology, 93(4), 1227-1234. doi:10.1152/japplphysiol.00187.2002

Bellet, S., Kershbaum, A., \& Finck, E. M. (1968). Response of free fatty acids to coffee and caffeine. Metabolism, 17(8), 702-707.

Benton, D., \& Owens, D. S. (1993). Blood glucose and human memory. Psychopharmacology, 113(1), 83-88. Springer.

Benton, D., \& Parker, P. Y. (1998). Breakfast, blood glucose, and cognition. The American Journal of Clinical Nutrition, 67(4), 772S-778S.

Benton, D., \& Sargent, J. (1992). Breakfast, blood glucose and memory. Biological Psychology, 33, 207-210.

Blomstrand, E., Hassmén, P., Ekblom, B., \& Newsholme, E. A. (1991). Administration of branched-chain amino acids during sustained exercise- effects on performance and on plasma concentration of some amino acids. European Journal of Applied Physiology and Occupational Physiology, 63(2), 83-88. Springer. 
Bridge, C. A., \& Jones, M. A. (2006). The effect of caffeine ingestion on $8 \mathrm{~km}$ run performance in a field setting. Journal of Sports Sciences, 24(4), 433-439. doi:10.1080/02640410500231496

Bruce, C. R., Anderson, M. E., Fraser, S. F., Stepto, N. K., Klein, R., HOPKINS, W. G., \& HAWLEY, J. A. (2000). Enhancement of 2000-m rowing performance after caffeine ingestion. Medicine and Science in Sports and Exercise, 32(11), 1958-1963.

Brunyé, T. T., Mahoney, C. R., Lieberman, H. R., Giles, G. E., \& Taylor, H. A. (2010). Acute caffeine consumption enhances the executive control of visual attention in habitual consumers. Brain and Cognition, 74(3), 186-192.

doi:10.1016/j.bandc.2010.07.006

Brunyé, T. T., Mahoney, C. R., Lieberman, H. R., \& Taylor, H. A. (2010). Caffeine modulates attention network function. Brain and Cognition, 72(2), 181-1888.

Elsevier Inc. doi:10.1016/j.bandc.2009.07.013

Bérubé-Parent, S., Pelletier, C., Doré, J., \& Tremblay, A. (2005). Effects of encapsulated green tea and Guarana extracts containing a mixture of epigallocatechin-3-gallate and caffeine on $24 \mathrm{~h}$ energy expenditure and fat oxidation in men. British Journal of Nutrition, 94(03), 432-436. doi:10.1079/BJN20051502

Casal, D. C., \& Leon, A. S. (1985). Failure of caffeine to affect substrate utilization during prolonged running. Medicine and Science in Sports and Exercise, 17(1), 174179.

Coles, K., \& Tomporowski, P. D. (2008). Effects of acute exercise on executive processing, short-term and long-term memory. Journal of Sports Sciences, 26(3), 333-344. doi:10.1080/02640410701591417

Copp, A. J., \& Greene, N. D. E. (2010). Genetics and development of neural tube defects. The Journal of Pathology, 220(2), 217-230. Wiley Online Library. doi:10.1002/path

Costill, D. L., Coyle, E., Dalsky, G., Evans, W., Fink, W., \& Hoopes, D. (1977). Effects of elevated plasma FFA and insulin on muscle glycogen usage during exercise. Journal of Applied Physiology, 43(4), 695-699.

Costill, D. L., Dalsky, G. P., \& Fink, W. J. (1978). Effects of caffeine ingestion on metabolism and exercise performance. Medicine and Science in Sports and Exercise, 10(3), 155-158. 
Coyle, E. F., Coggan, A. R., Hemmert, M. K., \& Ivy, J. L. (1986). Muscle glycogen utilization during prolonged strenuous exercise when fed carbohydrate. Journal of Applied Physiology, 61(1), 165-172.

Davis, J. M., Zhao, Z., Stock, H. S., Mehl, K. A., Buggy, J., \& Hand, G. A. (2003). Central nervous system effects of caffeine and adenosine on fatigue. American Journal of Physiology. Regulatory, Integrative and Comparative Physiology, 284(2), R399-404. doi:10.1152/ajpregu.00386.2002

Doan, B. K., Hickey, P. A., Lieberman, H. R., \& Fischer, J. R. (2006). Caffeinated Tube Food Effect on Pilot Performance During a 9-hour, Simulated Nighttime U-2 Mission. Aviation, Space, and Environmental Medicine, 77(10), 1034-1040.

Dragoo, K. R., Silvers, W. M., Johnson, K. E., \& Gonzalez, E. A. (2011). Effects of a Caffeine-Containing Transdermal Energy Patch on Aerobic and Anaerobic Exercise Performance. International Journal of Exercise Science, 4(2), 141-151.

Duncombe, W. G. (1964). The colorimetric micro-determination of non-esterified fatty acids in plasma. Clinica Chimica Acta, 9, 122-125.

Durlach, P. J. (1998). The effects of a low dose of caffeine on cognitive performance. Psychopharmacology, 140(1), 116-119.

Durlach, P. J., Edmunds, R., Howard, L., \& Tipper, S. P. (2002). A Rapid Effect of Caffeinated Beverages on Two Choice Reaction Time Tasks. Nutritional Neuroscience, 5(6), 433-442. doi:10.1080/1028415021000039211

Duvnjak-Zaknich, D. M., Dawson, B. T., Wallman, K. E., \& Henry, G. (2011). Effect of Caffeine on Reactive Agility Time When Fresh and Fatigued. Medicine and Science in Sports and Exercise, 43(8), 1523-1530. doi:10.1249/MSS.0b013e31821048ab

Eisenberg, F., Dayton, P. G., \& Burns, J. J. (1959). Studies on the Glucuronic Acid Pathway of Glucose Metabolism. The Journal of Biological Chemistry, 234(2), 250253.

Erickson, M. A., Schwarzkopf, R. J., \& McKenzie, R. D. (1987). Effects of caffeine, fructose, and glucose ingestion on muscle glycogen utilization during exercise. Medicine and Science in Sports and Exercise, 19(6), 579+583.

Essig, D., Costill, D. L., \& Van Handel, P. J. (1980). Effects of Caffeine Ingestion on Utilization of Muscle Glycogen and Lipid During Leg Ergometer Cycling. International Journal of Sports Medicine, 1(1), 86-90. 
Foster, C., Costill, D. L., \& Fink, W. J. (1979). Effects of preexercise feedings on endurance performance. Medicine and Science in Sports, 11(1), 1-5.

Fredholm, B. B., Chen, J.-F., Masino, S. a, \& Vaugeois, J.-M. (2005). Actions of adenosine at its receptors in the CNS: insights from knockouts and drugs. Annual review of pharmacology and toxicology, 45(30), 385-412. doi:10.1146/annurev.pharmtox.45.120403.095731

Geiß, K.-R., Jester, I., Falke, W., Hamm, M., \& Waag, K.-L. (1994). The effect of a taurine-containing drink on performance in 10 endurance-athletes. Amino Acids, (7), 45-56. doi:10.1016/0958-2118(94)90125-2

Giordano, D., Corrado, F., Santamaria, A., Quattrone, S., Pintaudi, B., Di Benedetto, A., \& D'Anna, R. (2011). Effects of myo-inositol supplementation in postmenopausal women with metabolic syndrome: a perspective, randomized, placebo-controlled study. Menopause, 18(1), 102-104. doi:10.1097/gme.0b013e3181e8e1b1

Graham, T.E., \& Spriet, L. L. (1991). Performance and metabolic responses to a high caffeine dose during prolonged exercise. Journal of Applied Physiology, 71(6), 2292-2298.

Graham, T.E., \& Spriet, L. L. (1995). Metabolic, catecholamine, and exercise performance responses to various doses of caffeine. Journal of Applied Physiology, 78(3), 867-874. Am Physiological Soc.

Graham, Terry E. (2001). Caffeine and Exercise. Sports Medicine, 31(11), 785-807.

Graham, Terry E., Helge, J. W., MacLean, D. A., Kiens, B., \& Richter, E. A. (2000). Caffeine ingestion does not alter carbohydrate or fat metabolism in human skeletal muscle during exercise. Journal of Physiology, 529(3), 837-847.

Haller, C. A., Jacob, P., \& Benowitz, N. L. (2005). Short-term metabolic and hemodynamic effects of ephedra and guarana combinations. Clinical Pharmacology and Therapeutics, 77(6), 560-571. doi:10.1016/j.clpt.2005.01.023

Hallman, M., Bry, K., Hoppu, K., Lappi, M., \& Pohjavuori, M. (1992). Inositol supplementation in premature infants with respiratory distress syndrome. New England Journal of Medicine, 326(19), 1233-1239. Mass Medical Soc.

Hargreaves, M., Costill, D. L., Fink, W., King, D., Fielding, R., \& others. (1987). Effect of pre-exercise carbohydrate feedings on endurance cycling performance. Medicine and science in sports and exercise, 19(1), 33. 
Haskell, C. F., Kennedy, D. O., Wesnes, K. A., Milne, A. L., \& Scholey, A. B. (2007). A double-blind, placebo-controlled, multi-dose evaluation of the acute behavioural effects of guaraná in humans. Journal of Psychopharmacology, 21(1), 65-70.

Heatherley, S. V., Hayward, R. C., Seers, H. E., \& Rogers, P. J. (2005). Cognitive and psychomotor performance, mood, and pressor effects of caffeine after 4, 6 and $8 \mathrm{~h}$ caffeine abstinence. Psychopharmacology, 178(4), 461-470. doi:10.1007/s00213005-2159-9

Heckman, M. A., Sherry, K., \& Gonzalez de Mejia, E. (2010). Energy Drinks: An Assessment of Their Market Size, Consumer Demographics, Ingredient Profile, Functionality, and Regulations in the United States. Comprehensive Reviews in Food Science and Food Safety, 9(3), 303-317. Wiley Online Library.

Hogervorst, E., Bandelow, S., Schmitt, J., Jentjens, R., Oliveira, M., Allgrove, J., Carter, T., et al. (2008). Caffeine Improves Physical and Cognitive Performance During Exhaustive Exercise. Medicine and Science in Sports and Exercise, 40(10), 18411851. doi:10.1249/MSS.0b013e31817bb8b7

Horne, J. A., \& Reyner, L. A. (2001). Beneficial effects of an "energy drink" given to sleepy drivers. Amino Acids, 20(1), 83-89.

Howard, M. A., \& Marczinski, C. A. (2010). Acute Effects of a Glucose Energy Drink on Behavioral Control. Experimental and Clinical Psychopharmacology, 18(6), 553561. doi:10.1037/a0021740

Hulston, C. J., \& Jeukendrup, A. E. (2008). Substrate Metabolism and Exercise Performance with Caffeine and Carbohydrate Intake. Medicine and Science in Sports and Exercise, 40(12), 2096-2104. doi:10.1249/MSS.0b013e318182a9c7

Ito, K., Arko, M., Kawaguchi, T., Kuwahara, M., \& Tsubone, H. (2009). The Effect of Subacute Supplementation of Taurine on Spatial Learning and Memory. Experimental Animals, 58(2), 175-180.

Ivy, John L., Kammer, L., Ding, Z., Wang, B., Bernard, J. R., Liao, Y.-H., \& Hwang, J. (2009). Improved Cycling Time-Trial Performance After Ingestion of a Caffeine Energy Drink. International Journal of Sport Nutrition and Exercise Metabolism, 19(1), 61-78.

Ivy, J.L., Costill, D. L., Fink, W. J., \& Lower, R. W. (1979). Influence of caffeine and carbohydrate feedings on endurance performance. Medicine and Science in Sports, 11(1), 6-11. 
Ivy, J.L., Miller, W., Dover, V., Goodyear, L. G., Sherman, W. M., Farrell, S., \& Williams, H. (1983). Endurance improved by ingestion of a glucose polymer supplement. Medicine and Science in Sports and Exercise, 15(6), 466-471.

Ivy, John L., Res, P. T., Sprague, R. C., \& Widzer, M. O. (2003). Effect of a Carbohydrate-Protein Supplement on Endurance Performance During Exercise of Varying Intensity. International Journal of Sport Nutrition and Exercise Metabolism, 13, 382-395. Human Kinetics.

James, J. E., \& Gregg, M. E. (2004). Hemodynamic effects of dietary caffeine, sleep restriction, and laboratory stress. Psychophysiology, 41(6), 914-923. doi:10.1111/j.1469-8986.2004.00248.x

Jeukendrup, A. E., \& Killer, S. C. (2010). The Myths Surrounding Pre-Exercise Carbohydrate Feeding. Annals of Nutrition and Metabolism, 57(Suppl 2), 18-25. doi:10.1159/000322698

Kennedy, D. O., \& Scholey, A. B. (2004). A glucose-caffeine “energy drink” ameliorates subjective and performance deficits during prolonged cognitive demand. Appetite, 42(3), 331-333. doi:10.1016/j.appet.2004.03.001

Kirwan, J. P., O’Gorman, D., \& Evans, W. J. (1998). A moderate glycemic meal before endurance exercise can enhance performance. Journal of Applied Physiology, 84(1), 53-59.

Kovacs, E. M. R., Stegen, J. H. C. H., \& Brouns, F. (1998). Effect of caffeinated drinks on substrate metabolism, caffeine excretion, and performance. Journal of Applied Physiology, 85(2), 709-715. Am Physiological Soc.

Lambourne, K., Audiffren, M., \& Tomporowski, P. D. (2010a). Effects of Acute Exercise on Sensory and Executive Processing Tasks. Medicine and Science in Sports and Exercise, 42(7), 1396-1402. doi:10.1249/MSS.0b013e3181cbee11

Lambourne, K., Audiffren, M., \& Tomporowski, P. D. (2010b). Effects of acute exercise on sensory and executive processing tasks. Medicine and science in sports and exercise, 42(7), 1396-402. doi:10.1249/MSS.0b013e3181cbee11

Laurent, D. (2000). Effects of Caffeine on Muscle Glycogen Utilization and the Neuroendocrine Axis during Exercise. Journal of Clinical Endocrinology \& Metabolism, 85(6), 2170-2175. doi:10.1210/jc.85.6.2170 
Lee, S.-T., Chu, K., Sim, J.-Y., Heo, J.-H., \& Kim, M. (2008). Panax Ginseng Enhances Cognitive Performance in Alzheimer Disease. Alzheimer Disease and Associated Disorders, 22(3), 222-226. doi:10.1097/WAD.0b013e31816c92e6

Lemaire, J. B., Wallace, J. E., Dinsmore, K., Lewin, A. M., Ghali, W. A., \& Roberts, D. (2010). Physician nutrition and cognition during work hours: effect of a nutrition based intervention. BMC Health Services Research, 10(241), 1-9. doi:10.1186/14726963-10-241

Lieberman, H. (2003). Nutrition, brain function and cognitive performance $\star$. Appetite, 40(3), 245-254. doi:10.1016/S0195-6663(03)00010-2

Lieberman, H. R. (2001). The Effects of Ginseng, Ephedrine, and Caffeine on Cognitive Performance, Mood and Energy. Nutrition Reviews, 59(4), 91-102.

Lieberman, H. R., Caruso, C. M., Niro, P. J., Adam, G. E., Kellogg, M. D., Nindl, B. C., \& Kramer, F. M. (2008). A double-blind, placebo-controlled test of $2 \mathrm{~d}$ of calorie deprivation: effects on cognition, activity, sleep, and interstitial glucose concentrations. The American Journal of Clinical Nutrition, 88(3), 667-676.

Lieberman, H. R., Falco, C. M., \& Slade, S. S. (2002). Carbohydrate administration during a day of sustained aerobic activity improves vigilance, as assessed by a novel ambulatory monitoring device, and mood. The American Journal of Clinical Nutrition, 76(1), 120-127.

Lieberman, H. R., Tharion, W. J., Shukitt-Hale, B., Speckman, K. L., \& Tulley, R. (2002). Effects of caffeine, sleep loss, and stress on cognitive performance and mood during U.S. Navy SEAL training. Psychopharmacology, 164(3), 250-261. doi:10.1007/s00213-002-1217-9

Lockwood, C. M., Moon, J. R., Smith, A. E., Tobkin, S. E., Kendall, K. L., Graef, J. L., Cramer, J. T., et al. (2010). Low-Calorie Energy Drink Improves Physiological Response to Exercise in Previously Sedentary Men: a Placebo-Controlled Efficacy and Safety Study. The Journal of Strength and Conditioning Research, 24(8), 22272238.

Lopes, J. M., Aubier, M., Jardim, J., Aranda, J. V., \& Macklem, P. T. (1983). Effect of caffeine on skeletal muscle function before and after fatigue. Journal of Applied Physiology, 54(5), 1303-1305.

McMorris, T., \& Graydon, J. (1997). The effect of exercise on cognitive performance in soccer-specific tests. Journal of Sports Sciences, 15(5), 459-468. Taylor \& Francis. 
McMorris, T., Myers, S., MacGillivary, W. W., Sexsmith, J. R., Fallowfield, J. E., Graydon, J., \& Forster, D. (1999). Exercise, plasma catecholamine concentrations and decision-making performance of soccer players on a soccer-specific test. Journal of Sports Sciences, 17(8), 667-676. Taylor \& Francis.

McMorris, T., \& Rayment, T. (2007). Short-duration, high-intensity exercise and performance of a sports-specific skill: a preliminary study. Perceptual and Motor Skills, 105(2), 523-530.

McMorris, T., Sproule, J., Draper, S., \& Child, R. (2000). Performance of a psychomotor skill following rest, exercise at the plasma epinephrine threshold and maximal intensity exercise. Perceptual and Motor Skills, 91(2), 553-562. Ammons Scientific.

McMorris, T., Sproule, J., Turner, A., \& Hale, B. J. (2011). Acute, intermediate intensity exercise, and speed and accuracy in working memory tasks: A meta-analytical comparison of effects. Physiology and Behavior, 102, 421-428. Elsevier Inc. doi:10.1016/j.physbeh.2010.12.007

Mets, M. A. J., Ketzer, S., Blom, C., van Gerven, M. H., van Willigenburg, G. M., Olivier, B., \& Verster, J. C. (2011). Positive effects of Red Bull® Energy Drink on driving performance during prolonged driving. Psychopharmacology, 214(3), 737 745. doi:10.1007/s00213-010-2078-2

Noma, A., Okabe, H., \& Kita, M. (1973). A new colorimetric micro-determination of free fatty acids in serum. Clinica Chimica Acta, 43(3), 317-320. doi:10.1016/00098981(73)90468-3

O'Brien, M., \& Koo, J. (2006). The Mechanism of Lithium and Beta-Blocking Agents in Inducing and Exacerbating Psoriasis. Journal of Drugs in Dermatology, 5, 426-432. Strategic Communication in Dermatology.

Papadelis, C., Kourtidou-Papadeli, C., Vlachogiannis, E., Skepastianos, P., Bamidis, P., Maglaveras, N., \& Pappas, K. (2003). Effects of mental workload and caffeine on catecholamines and blood pressure compared to performance variations. Brain and Cognition, 51(1), 143-154. doi:10.1016/S0278-2626(02)00530-4

Petkov, V. D., Belcheva, S., \& Petkov, V. V. (2003). Behavioral Effects of Ginkgo biloba L., Panax ginseng C.A. Mey. and Gincosan ${ }^{\circledR}$. The American Journal of Chinese Medicine, 31(6), 841-855.

Pierno, S., De Luca, A., Camerino, C., Huxtable, R. J., \& Camerino, D. C. (1998). Chronic Administration of Taurine to Aged Rats Improves the Electrical and 
Contractile Properties of Skeletal Muscle Fibers. The Journal of Pharmacology and Experimental Therapeutics, 286(3), 1183-1190.

Pollitt, E., Cueto, S., \& Jacoby, E. R. (1998). Fasting and cognition in well- and undernourished schoolchildren: a review of three experimental studies. The American Journal of Clinical Nutrition, 67(4), 779S-784S.

Reay, J.L., Kennedy, D. O., \& Scholey, A. B. (2006). The glycaemic effects of single doses of Panax ginseng in young healthy volunteers. British Journal of Nutrition, 44(96), 639-642. doi:10.1079/BJN20061904

Reay, Jonathon L., Scholey, A. B., \& Kennedy, D. O. (2010). Panax ginseng (G115) improves aspects of working memory performance and subjective ratings of calmness in healthy young adults. Human Psychopharmacology: Clinical and Experimental, (25), 462-471. doi:10.1002/hup

Rivers, W. H. R., \& Webber, H. N. (1907). The action of caffeine on the capacity for muscular work. The Journal of Physiology, (36), 33-47.

Rollo, I., \& Williams, C. (2010). Influence of ingesting a carbohydrate-electrolyte solution before and during a 1-hour run in fed endurance-trained runners. Journal of Sports Sciences, 28(6), 593-601. doi:10.1080/02640410903582784

Sale, C., Harris, R. C., Delves, S., \& Corbett, J. (2006). Metabolic and physiological effects of ingesting extracts of bitter orange, green tea and guarana at rest and during treadmill walking in overweight males. International Journal of Obesity, 30, 764773. doi:10.1038/sj.ijo.0803209

Sanabria, D., Morales, E., Luque, A., Gálvez, G., Huertas, F., \& Lupiañez, J. (2011). Effects of acute aerobic exercise on exogenous spatial attention. Psychology of Sport and Exercise, 12, 570-574. Elsevier Ltd. doi:10.1016/j.psychsport.2011.04.002

Santini, A., Ferracane, R., Mikušová, P., Eged, Š., Šrobárová, A., Meca, G., Mañes, J., et al. (2011). Influence of different coffee drink preparations on ochratoxin A content and evaluation of the antioxidant activity and caffeine variations. Food Control, 22, 1240-1245. doi:10.1016/j.foodcont.2011.01.024

Schneiker, K. T., Bishop, D., Dawson, B., \& Hackett, L. P. (2006a). Effects of Caffeine on Prolonged Intermittent-Sprint Ability in Team-Sport Athletes. Medicine and Science in Sports and Exercise, 38(3), 578-585.

doi:10.1249/01.mss.0000188449.18968.62 
Schneiker, K. T., Bishop, D., Dawson, B., \& Hackett, L. P. (2006b). Effects of caffeine on prolonged intermittent-sprint ability in team-sport athletes. Medicine and science in sports and exercise, 38(3), 578-85. doi:10.1249/01.mss.0000188449.18968.62

Scholey, A. B., \& Kennedy, D. O. (2002). Acute, dose-dependent cognitive effects of Ginkgo biloba, Panax ginseng and their combination in healthy young volunteers: differential interactions with cognitive demand. Human Psychopharmacology, 17(1), 35-44. doi:10.1002/hup.352

Scholey, A. B., \& Kennedy, D. O. (2004). Cognitive and physiological effects of an "energy drink": an evaluation of the whole drink and of glucose, caffeine and herbal flavouring fractions. Psychopharmacology, 176(3-4), 320-30. doi:10.1007/s00213004-1935-2

Seidl, R., Peyrl, A., Nicham, R., \& Hauser, E. (2000). A taurine and caffeine-containing drink stimulates cognitive performance and well-being. Amino Acids, 19, 635-642.

Sherman, W., Brodowicz, G., \& Wright, D. (1989). Effects of 4 h preexercise carbohydrate feedings on cycling performance. Medicine and science.

Sherman, W. M., Brodowicz, G., Wright, D. A., Allen, W. K., Simonsen, J., \& Dernbach, A. (1989). Effects of $4 \mathrm{~h}$ preexercise carbohydrate feedings on cycling performance. Medicine and Science in Sports and Exercise, 21(5), 598-604.

Skinner, T. L., Jenkins, D. G., Coombes, J. S., Taaffe, D. R., \& Leveritt, M. D. (2010). Dose Response of Caffeine on 2000-m Rowing Performance. Medicine and Science in Sports and Exercise, 42(3), 571-576. doi:10.1249/MSS.0b013e3181b6668b

Smit, H. J., Cotton, J. R., Hughes, S. C., \& Rogers, P. J. (2004). Mood and Cognitive Performance Effects of "Energy" Drink Constituents: Caffeine, Glucose and Carbonation. Nutritional Neuroscience, 7(3), 127-139. doi:10.1080/10284150400003041

Smit, H. J., \& Rogers, P. J. (2000). Effects of low doses of caffeine on cognitive performance, mood and thirst in low and higher caffeine consumers. Psychopharmacology, 152(2), 167-173. doi:10.1007/s002130000506

Smith, A. P. (2005). Caffeine at work. Human Psychopharmacology, 20(6), 441-445. doi:10.1002/hup.705

Spriet, L. L., MacLean, D. A., Dyck, D. J., Hultman, E., Cederblad, G., \& Graham, T. E. (1992). Caffeine ingestion and muscle metabolism during prolonged exercise in humans. The American Journal of Physiology, 262, E891-E898. 
Tamura, S., Tsutsumi, S., Ito, H., Nakai, K., \& Masuda, M. (1968). Effects of glucuronolactone and the other carbohydrates on the biochemical changes produced in the living body of rats by hard exercise. Japanese Journal of Pharmacology, 18, 30-38.

Tanaka, H. (2001). Age-predicted maximal heart rate revisited. Journal of the American College of Cardiology, 27(1), 1406-156. doi:10.1016/S0735-1097(00)01054-8

Tarnopolsky, M., \& Cupido, C. (2000). Caffeine potentiates low frequency skeletal muscle force in habitual and nonhabitual caffeine consumers. Journal of Applied Physiology, 89, 1719-1724. Am Physiological Soc.

Thornton, G. R., Holck, H. G. O., \& Smith, E. L. (1939). The effect of benzedrine and caffeine upon performance in certain psychomotor tasks. The Journal of Abnormal and Social Psychology, 34(1), 96-113. doi:10.1037/h0062063

Tomporowski, P. D. (2003). Effects of acute bouts of exercise on cognition. Acta Psychologica, 112(3), 297-324.

Waldeck, B. (1973). Sensitization by Caffeine of Central Catecholamine Receptors. Journal of Neural Transmission, 34, 61-72.

Walsh, A. L., Gonzalez, A. M., Ratamess, N. A., Kang, J., \& Hoffman, J. R. (2010). Improved time to exhaustion following ingestion of the energy drink Amino Impact $^{\mathrm{TM}}$. Journal of the International Society of Sports Nutrition, 7(14), 1-6. doi:10.1186/1550-2783-7-14

Warburton, D. M., Bersellini, E., \& Sweeney, E. (2001). An evaluation of a caffeinated taurine drink on mood, memory and information processing in healthy volunteers without caffeine abstinence. Psychopharmacology, 158(3), 322-328.

doi:10.1007/s002130100884

Warskulat, U., Flögel, U., Jacoby, C., Hartwig, H.-G., Thewissen, M., Merx, M. W., Molojavyi, A., et al. (2004). Taurine transporter knockout depletes muscle taurine levels and results in severe skeletal muscle impairment but leaves cardiac function uncompromised. The FASEB Journal, 18(3), 577-579. doi:10.1096/fj.03-0496fje

Weir, J., Noakes, T. D., Myburgh, K., \& Adams, B. (1987). A high carbohydrate diet negates the metabolic effects of caffeine during exercise. Medicine and Science in Sports and Exercise, 19(2), 100-105. 
Welsh, R. S., Davis, J. M., Burke, J. R., \& Williams, H. G. (2002). Carbohydrates and physical/mental performance during intermittent exercise to fatigue. Medicine and Science in Sports and Exercise, 34(4), 723-731.

Wesensten, N. J., Belenky, G., Kautz, M. A., Thorne, D. R., Reichardt, R. M., \& Balkin, T. J. (2002). Maintaining alertness and performance during sleep deprivation: modafinil versus caffeine. Psychopharmacology, 159(3), 238-247. doi:10.1007/s002130100916

Wiles, J.D., Bird, S. R., Hopkins, J., \& Riley, M. (1992). Effect of caffeinated coffee on running speed, respiratory factors, blood lactate and perceived exertion during 1500$\mathrm{m}$ treadmill running. British Journal of Sports Medicine, 26(2), 116-120. BMJ Publishing Group Ltd and British Association of Sport and Exercise Medicine.

Wiles, Jonathan D., Coleman, D., Tegerdine, M., \& Swaine, I. L. (2006). The effects of caffeine ingestion on performance time, speed and power during a laboratory-based $1 \mathrm{~km}$ cycling time-trial. Journal of Sports Sciences, 24(11), 1165-1171. doi:10.1080/02640410500457687

Yaspelkis, B. B., Patterson, J. G., Anderla, P. A., Ding, Z., \& Ivy, J. L. (1993). Carbohydrate supplementation spares muscle glycogen during variable-intensity exercise. Journal of Applied Physiology, 75(4), 1477-1485. 